\title{
A Qualitative Study of the Biophysics of Tooth Movement by Means of a Three-Dimensional Analog
}

\author{
Edgar Francis Geigel \\ Loyola University Chicago
}

Follow this and additional works at: https://ecommons.luc.edu/luc_theses

Part of the Dentistry Commons

\section{Recommended Citation}

Geigel, Edgar Francis, "A Qualitative Study of the Biophysics of Tooth Movement by Means of a ThreeDimensional Analog" (1965). Master's Theses. 1973.

https://ecommons.luc.edu/luc_theses/1973

This Thesis is brought to you for free and open access by the Theses and Dissertations at Loyola eCommons. It has been accepted for inclusion in Master's Theses by an authorized administrator of Loyola eCommons. For more information, please contact ecommons@luc.edu.

Copyright @ 1965 Edgar Francis Geigel 
A QUALITATIVE STUDY OF THE BIOPHYSICS OF

TOOTH MOVEMENT BY MEANS OF A

THREE-DIMENSIONAL ANALOG

\section{by}

Edgar Francis Geige1

A Thesis Submitted to the Faculty of the Graduate Schoo1 of Loyola University in Partial Fulfillment of the Requirements for the Degree of

Master of Science

JUNE

1965

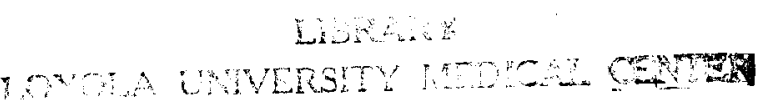




\section{LIFE}

Edgar Francis Geige1 was born in New York City, New York, on June 9th, 1936. He received his secondary education at A11 Hallows Institute in the Bronx, New York, and upon graduating in 1954, he enrolled in New York University for predental studies.

In September, 1956, he entered the University of Pennsylvania School of Dentistry, Philadelphia, Pennsylvania, and graduated in June, 1960, with the degree of Doctor of Dental Surgery.

From 1960 to 1963 the author served as a commissioned dental officer in the United States Army Reserve on active duty in the Panama Canal Zone. Since June of 1963 he has been enrolled in the Graduate School of Loyola University (Chicago, Illinois) working toward a Master of Science Degree in Ora1 Biology.

He is married and has a daughter and a son.

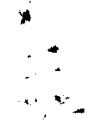




\section{ACKNOWLEDGEMENTS}

I wish to thank the Loyola-Jarabak Orthodontic Foundation for the contribution of funds which made this research possible.

I also gratefully acknowledge and sincerely thank my advisor and teacher, Joseph R. Jarabak, D.D.S., M.S., Ph.D., Professor of Orthodontics, Loyola University, for his invaluable guidance during this investigation and for the inspiration I received from his boundless enthusiasm for the profession.

I am most grateful to James A. Fizze11, B.S. in E.E., for his suggestions and technical advice in the preparation of this thesis, for his assistance in the construction of the analogs, and for his aid in the analysis of the data. I fully recognize that without his enlightenment, help and supervision, this work could not have been undertaken and completed.

I am thankful to D.C. Bowman, D.D.S., Ph.D., and to A.C. Claus, B.S., $\mathrm{Ph}$.D., for serving as members of my advisory board.

Final1y, I am indeed greatly indebted to my wife, Rita, for the love, patience and encouragement with which she has abundantly endowed me during the pursuance of this endeavor. 
TABLE OF CONTENTS

Chapter

Page

I. INTRODUCTION AND GENERAL CONSIDERATIONS. . . . . . . . . 1

II. STATEMENT OF THE PROBLEM . . . . . . . . . . . . . . . . 6

III. REVIEW OF THE LITERATURE . . . . . . . . . . . . . . . . 7

A. Biomechanics of tooth movement . . . . . . . . 8

B. Tooth movement as a function of pressure . . . . . 15

C. Use of models to study tooth movement. . . . . . . . 22

IV. PROCEDURE. . . . . . . . . . . . . . . . . . . . 44

A. Selection of the model tooth . . . . . . . . . . 44

B. Construction of the model alveolus . . . . . . . . . 46

C. Construction of the periodontal 1igament analog. . . 52

D. Methods of study . . . . . . . . . . . . . . . 56

E. Applying pure couples to the analog. . . . . . . . 59

F. Applying tipping forces to the analog. . . . . . . . 62

G. Producing bodily movement of the model tooth . . . . 64

V. EXPERIMENTAL RESULTS . . . . . . . . . . . . . . 66

A. Appraisal of tipping produced by pure couples. . . . 66

B. Appraisal of tipping produced by single forces . . . 67

1. The effects of changing force magnitude. . . . . 67

2. The effects of changing point of application . . 70

3. Statistical analysis of the data. . . . . . 72

C. Appraisal of bodily movement produced by a force and a couple... . . . . . . . . . . 77

VI. DISCUSSION . . . . . . . . . . . . . . . . . . . . 80

A. Location of the centroid of effective root area. - . 81

B. Assessment of tipping. . . . . . . . . . . . 86

C. Assessment of bodily movement. . . . . . . . . . 93

D. Biophysics of tooth movement . . . . . . . . . . 99 
VII. SUMMARY AND CONCLUSIONS. . . . . . . . . . . 109

APPENDICES . . . . . . . . . . . . . . 113

GLOSSARY . . . . . . . . . . . . . . . 118

BIBLIOGRAPHY . . . . . . . . . . . . . . 121 


\section{LIST OF TABLES}

Table

Page

I. ANALYSIS OF VARIANCE (CROWN DEFLECTIONS) . . . . . . . . . 73

II. ANALYSIS OF VARIANCE (ROOT DEFLECTIONS) . . . . . . . . . . 75 


\section{LIST OF FIGURES}

Figure

Page

1. THEORIES OF TIPPING. . . . . . . . . . . . . 10

2. POST IN THE GROUND EXPERIMENT BY CASE. . . . . . . . . . . 24

3. MATCHSTICK IN PLASTER EXPERIMENT BY SCHWARZ. . . . . . . . 25

4. MATHEMATICAL MODEL USED BY SCHWARZ . . • • . . . . . • . . 26

5. MATHEMATICAL MODEL USED BY BAUER AND LANG. . • . . . . . . 27

6. STANDARD MATHEMATICAI MODELS DEVISED BY FICKEL . • • • . . 29

7. METAL PIECES FROM EXPERIMENT BY FICKEL TO DETERMINE CENTER OF ROTATION. • . . . . . . . . . . . 30

8. APPARATUS USED BY ZAK FOR PHOTO-ELASTIC ANALYSIS OF THE MECHANICS OF TOOTH MOVEMENT . . . . . . . . . . . 34

9. DIAGRAMS DEVELOPED BY HAY ILLUSTRATING PRESSURES

ON A MEMBRANE SURROUNDING A CONE-SHAPED ROOT . • • • . • 36

10. WOODEN MODEL USED BY HAACK AND WEINSTEIN TO DEMONSTRATE SPECIFIC FORCE SYSTEMS AND TOOTH MOVEMENTS . . . . . . . 39

11. MATHEMATICAL MODEL USED BY JARABAK AND FIZZELL TO STUDY PURE TRANSLATION. . . . . . . . . . . . . . . . 41

12. CHIPBOARD MODEL USED BY DEMPSTER AND DUDDLES TO STUDY TOOTH STATICS AND EQUILIBRIUM . . . . . . . . . 42

13. PLASTIC MODEL OF A LOWER LEFT CANINE . . . . . . . . . . . 45

14. MOLD FOR CASTING THE ALVEOLUS. . . . . . . . . . . . . 47

15. MODEL OF THE ROOT USED TO FORM THE INSIDE SHAPE OF THE ALVEOLUS. . . . . . . . . . . . . . 50

16. METHOD OF CASTING THE ALVEOLUS IN TWO STAGES . . . . . . . 51 
17. RUBBER ANALOG OF THE PERIODONTAL LIGAMENT

AND DIAGRAM OF A LONGITUDINAL CROSS-SECTION. . . . . . . . 53

18. PLEXIGLAS MOLDS USED TO FORM THE ANALOG

PERIODONTAL LIGAMENT . . . . . . . . . . . . . . 55

19. MODEL TOOTH WITH ANALOG PERIODONTAL LIGAMENT

CEMENTED TO THE ROOT SURFACE . . . . . . . . . . . . . . 57

20. COMPLETED ANALOG MOUNTED ON PLYWOOD ASSEMBLY

WITH CROWN AND ROOT DIAL INDICATORS. . . . . . . . . . 58

21. DIAGRAM OF HEIGHTS OF IMPORTANT POINTS ON THE MODEL TOOTH. . . . . . . . . . . . . . . . 60

22. DIAGRAM OF APPARATUS USED TO APPLY PURE COUPLES TO THE MODEL TOOTH . . . . . . . . . . . . . . . . . 61

23. GRAPH OF APPARENT CENTER OF TIPPING VS. TIPPING COUPLE . . 68

24. GRAPH OF CROWN AND ROOT DEFLECTIONS VS. TIPPING FORCE. . . 69

25. GRAPH OF APPARENT CENTER OF TIPPING VS. TIPPING FORCE. . . 71

26. GRAPH OF DISTRIBUTION OF EXPERIMENTAL ERROR. • • • • • • 76

27. VECTOR DIAGRAM AND EQUATIONS OF EQUILIBRIUM

REPRESENTING THE FORCE SYSTEM NECESSARY TO PRODUCE

BODILY MOVEMENT OF THE MODEL TOOTH . . . . . . . . . . . 78

28. GRAPH OF CROWN AND ROOT DEFLECTIONS

VS. MOTIVATING FORCE, $\mathrm{F}_{\mathrm{m}}$. . . . . . . . . . . . . . 79 


\section{CHAPTER I}

\section{INTRODUCTION AND GENERAL CONSIDERATIONS}

The patterns of force that act on a tooth are more complex than is generally believed. During an entire life span the teeth in both dental arches are constantly being subjected to a great variety of complex forces, pressures and stresses. First there is the little understood force of eruption that acts on each tooth during the stages of its root formation. Then there are pressures being applied continuously to the crowns of the teeth by the surrounding musculature and the tongue. During the functions of occluding of the teeth and masticating of food, forces of varying magnitudes and directions are intermittently being applied to the teeth in both arches. These forces produce a net horizontal component in the anterior direction (the anterior component of forces) which drives the teeth forward or toward the mid-1ine. Even when a tooth is isolated from proximal contacts in the arch and out of contact with an antagonist, there is still the force of gravity and the pressure of the atmosphere acting on it. Thus, the forces, pressures and stresses which act on teeth are complex in nature and many in number. Evidently they are all in balance since the teeth do occupy positions of seeming equilibrium.

Orthodontic appliances also deliver forces to the crowns of teeth when they are in a state of elastic deformation. The magnitude and 
direction of these forces can be predetermined and controlled rather precisely in many instances. Yet the precise manner in which the forces applied to the crowns of teeth introduce stresses in the periodontal ligament has not been clearly established. In the light of recent investigations, however, it is now rather evident that it is the distribution of forces against the alveolar walls, and not coronal forces, that determines the patterns of alveolar bone resorption and apposition that make orthodontic tooth movements possible. The actual manner in which forces are applied to the crowns of the teeth is important only to the extent that it relates to the pressures and tensions that are exerted against the wall of each a1veolus.

Since the roots of teeth are of different lengths and different morphological configurations, the surface area of each root differs from tooth to tooth. Thus, it follows logically that the surface area of alveolar wall surrounding each root will vary accordingly in the different teeth. If this is the case, then equal forces applied to teeth with different size roots cannot be distributed equally to the walls of the alveoli. This is apparent from the formula for pressure:

$$
\text { Pressure }=\frac{\text { Force }}{\text { Unit Area }} \text {. }
$$

If the forces applied to the crowns of teeth are identical for two given situations but the root surface areas are different, then the inverse relationship between pressure and area will manifest itself. That is, the tooth with greater root surface area will exert less pressure against the wall of its alveolus (through the periodontal ligament), while the tooth 
with lesser root surface area will develop greater pressure against the socket wall.

It seems logical, then, to think of orthodontic tooth movement as being the result of pressure against the alveolar bone, stimulating osteoclastic activity and bone resorption. This must be so since orthodontic forces applied to the crowns of teeth are immediately distributed to the roots where they are resisted. But the values of these pressures cannot be calculated simply by dividing the sum of the forces acting on a tooth by its total root surface area because the entire root surface is not involved at any one time in resisting the movement that the forces on the crown are tending to produce.

Orthodontic literature is replete with articles concerning the application of forces to teeth and the biologic reactions to these forces within the periodontal environment. Yet little attention has been given to an analytical evaluation of all the forces acting on a particular tooth at a particular time under a particular set of circumstances. To achieve a clearer perspective and more accurate orientation toward orthodontic problems as they relate to force distribution, research must be directed toward the determination of the "effective root surface area" of each tooth and "effective root pressure" under various conditions of force application. In the most recent literature available on this subject, "effective root surface area" is defined as the projected area of the root of a tooth on the pressure side, and "effective root pressure" is defined as the required pressure needed to start the tooth moving (Jarabak and Fizze11, 1963). 
One of the most convenient ways of studying the distribution of forces through the roots of the teeth to the alveolar walls is by the use of free-body diagrams. For years, investigators have been using models to represent different teeth and their respective alveoli. Some have used simple mechanical models while others have devised and studied mathematical models. With these models they have been able to demonstrate more realistically many of the forces acting in one plane on both the crown and the root of a tooth in a situation of equilibrium. This has been research in the right direction, but only on a smal1 scale when one considers that, in the complete dentition, there are twenty-two roots which transmit forces to the body of the mandible and up to thirty roots which do the same to both maxillae. Each of these roots has its own size and shape. In addition, root fusions and aberrant root formations are common and these present mechanical peculiarities of their own.

The free-body diagrams used in most of the previous investigations have an inherent 1 imitation in that they show only the forces that act in one plane of space and, hence, illustrate only the movements that occur in two dimensions. Clinically, however, a tooth that is being moved orthodontically responds to the application of forces by moving in three planes of space, and therefore, three free-body diagrams might be needed to show the forces.

From the foregoing explanation of current knowledge concerning orthodontic tooth movements it can be concluded that there is a real, but unknown, value of root pressure that initiates direct resorption of alveolar 
bone. A corollary to this observation is that there must also be a real, but unknown, subliminal pressure below which all movement ceases, and a supramaximal pressure which will cause undermining resorption. 
CHAPTER II

\section{STATEMENT OF THE PROBLEM}

The purpose of this project is to design and construct an enlarged, proportional, anatomical model of a tooth and its alveolus which will allow a qualitative evaluation of the tooth movements that occur when various force systems are applied to the crown. The force systems that will be applied to the three-dimensional model will be similar to those applied to the teeth clinically, differing only in that the magnitudes will be greater on the enlarged model. The movement that results under the influence of each force system will be studied empirically and the apparent center of tipping will be calculated mathematically. It is believed that there is a parallel between the movement of the model tooth within its plastic alveolus and that which occurs within the limits of the socket of a tooth immediately after engaging an active orthodontic appliance onto a tooth, that is, before any resorption has had a chance to take place.

It is hoped that this study will illustrate the analytical thinking that must be pursued if one is to ultimately achieve a true understanding of the biophysics of tooth movement. 
CHAPTER III

\section{REVIEW OF THE LITERATURE}

Biophysics is defined as that branch of physics which deals with living matter. It is the study of biologic function, structure, and organization in relation to and by the methods of physics. Obviously then, the biophysics inherent in tooth movement is a subject with many ramifications. When one undertakes a study of this subject by means of three-dimensional models, still other considerations are introduced which must be investigated thoroughly prior to experimentation.

Because of the complex character of the problem, the review of the 1iterature has been conducted with three specific and distinct purposes in mind: (1) to find out what attempts have been made in the past to explain the nature of orthodontic tooth movements and the precise mechanisms by which they are brought about (studies of this sort were formerly classed under the term "biomechanics of tooth movement"), (2) to discover whether any studies have been carried out in the past that define tooth movement as a function of pressure or stresses rather than as a function of force application, and (3) to determine whether models have ever been employed to study the biophysics of tooth movement. Accordingly, this review is reported in three sections. 


\section{A. Biomechanics of Tooth Movement}

When it was first discovered that teeth could be moved through alveolar bone, many dentists began to practice what soon became the clinical art of moving teeth in order to correct certain irregularities of the dental arches. Only as a result of the efforts of a few scientific thinkers was orthodontics advanced from purely mechanical considerations to a more professional and scientific endeavor which also placed significance on the biologic principles involved in orthodontic tooth movements. Biomechanics thus came into being as the science which related mechanics and biologic findings to the correction of malocclusions of teeth. This section of the review of the literature reports on those studies, done in the past, that relate to the manner in which teeth move under the influence of orthodontic forces, and those studies which give an analysis of the force systems used to bring about tooth movements.

Sandstedt (1904, 1905) was the first investigator to employ a histologic technique to examine orthodontic tooth movement. His experiments involved tipping the incisors of a dog lingually by means of a continuous archwire which was tightened with nuts every day for three weeks. From histologic sections that were made after the animals had been sacrificed, he was able to explain WHY it is possible to move teeth through alveolar bone, and he described the process which he called "undermining resorption." But he delved into the field of biomechanics when he described How the teeth had moved. He observed that the crown of each tooth had moved in the direction of the force while the apex of the root had moved in the 
opposite direction. Thus, he reasoned that in tooth movements we are dealing with a "double-armed lever", the fulcrum being at about the middle of the root (Figure $1 \mathrm{~A}$ ).

Angle (1907) did not agree with the explanation of tipping given by Sandstedt and in his textbook he deplored the comparison of a tooth in its alveolus to a stake in the ground. In his chapter dealing with tissue changes incident to tooth movement he stated that there is little displacement of the apex because of the greater resistance offered by the thickened bone in that area and by the "innumerable fibers that encapsule the apex."

Oppenheim (1911) was the first to employ a primate (baboon) for his studies of tooth movement. He applied a spring arch with ligatures for a period of forty days, leaving one-half of the jaw as a control. His findings differed sharply from those of Sandstedt (Figure 1B).

Oppenheim explained that his preparations showed bone changes almost throughout the entire length of the root, decreasing in intensity from the alveolar border to the root apex. In the area of immediate proximity to the root apex he found that no changes in the bone were observable. He concluded, therefore, that the tooth represents a "one-armed lever" with the apex of the root serving as the pivot point or center of rotation. He stated further that the point of view held by several authors, that the tooth represents a "two-armed lever", was incorrect.

Johnson, Appleton and Rittershofer (1926) conducted their research of tooth movement on two Macaque Rhesus monkeys using the labio-1ingual technique. The experiment (which lasted for twenty-six days in one animal 


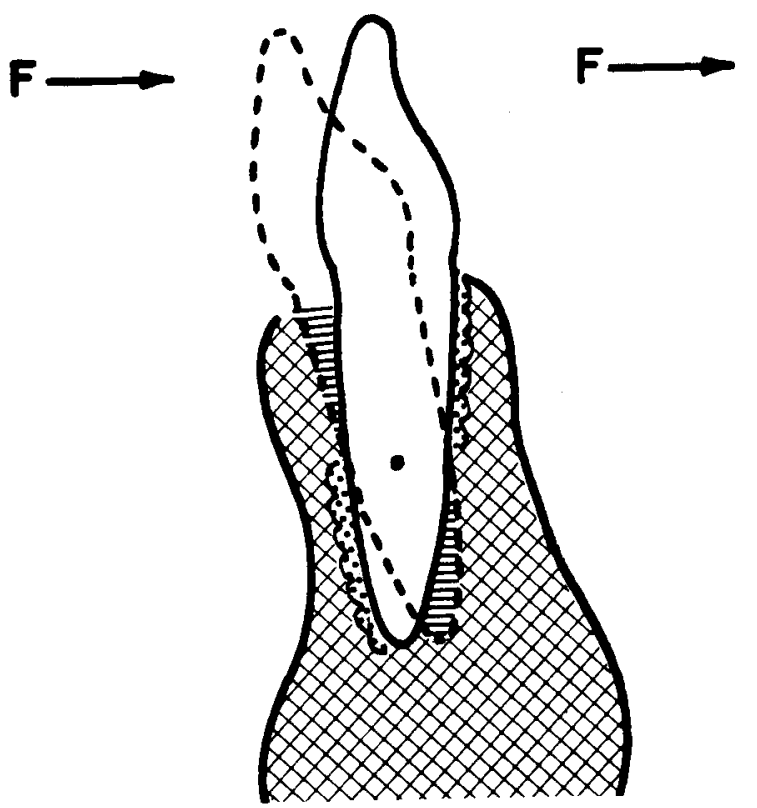

A

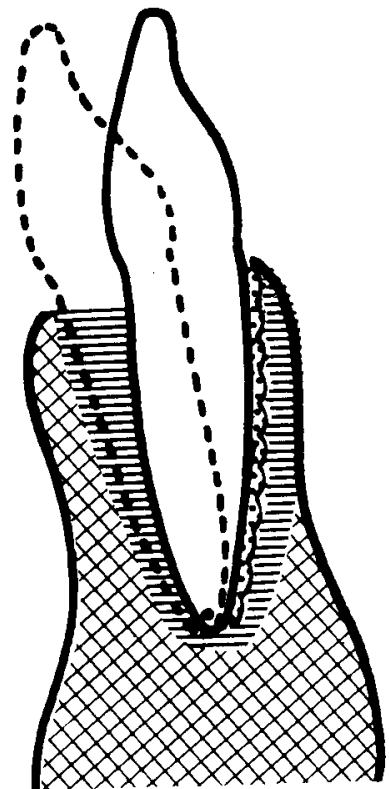

$B$

FIGURE 1

THEORIES OF TIPPING

Figure 1A. Sandstedt $(1904,1905)$ states that the force, F, produces a tilting around an axis which lies a little apically from the center of the root. Thus two regions of pressure and pull arise lying diametrically opposite to each other. In the regions of pressure the old alveolar bone is resorbed (jagged 1ine); in the regions of pull new bone is added (horizontal shading). The cross-hatching shows alveolar bone without transformation.

Figure 1B. Oppenheim (1911) states that a tooth moved by the force, F, tilts around an axis which lies at the apex. Therefore, there is only one side of pressure and one side of pull. On both sides, the alveolar bone changes into a transitional spongy bone (horizontal shading). On the side of pressure, this newly formed transitional bone is resorbed (jagged 1ine); on the side of pull new bone is added. The cross-hatching shows the untransformed alveolar bone at a greater distance from the moved tooth. 
and forty days in the other) demonstrated resorption and deformation of the root apex. In addition, they described the nature of the movement as being a tipping action in which the apex moved in an opposite direction to that of the crown. Their specimens demonstrated that the moved tooth acted like a "two-armed lever" and that the fulcrum was in about the middle of the root. This was in accord with Sandstedt's findings and contrary to what Oppenheim had said.

Schwarz (1928) subjected monkeys' teeth to horizontal and oblique forces and analyzed the "tilting" of the tooth that occurred within the alveolus. He found that in tipping a single rooted tooth, the center of rotation always lies "somewhere in the apical half of the clinical root, and nearer to the middle of the root than the apex."

Kronfeld (1931) confirmed the results of Schwarz's experiment by studying histologic sections of human teeth made from the jaws of a 38 year old man shortly after he died. He made very precise measurements of the widths of the periodontal spaces and showed that a tooth exposed to a strong horizontal stress (tipping force) had the minimum width at the middle of the alveolus and more than double the width toward the upper and lower ends of the root. He felt that this fact clearly established that the location of the fulcrum of a moving tooth is in the middle of the root. Storey and Smith (1952) studied the question of whether there was an optimum force that would cause teeth to move without damage. Through the use of very accurate measuring devices, they were able to determine the distance a tooth moved under the influence of a known force application. 
The experiments involved moving canine teeth distally into first premolar extraction sites. A "light" spring and a "heavy" spring were used to deliver the different, predetermined forces to the canine teeth. The TYPE of movement that occurred in every instance was described as "tipping approximately about the apical one-third of the root."

Graber (1961) also reported several observations and theories concerning the mechanics involved in tooth movements. He wrote in his textbook that:

A tipping force of moderate intensity sets up a fulcrum at about one-third the way up the root from the apex. But several factors influence the position of the fulcrum. One is the point of application of force. The closer to the incisal edge the point of force application, the greater the distance of the fulcrum from the apex. The same fulcrum reaction takes place with an increase in the intensity of force application. Heavy forces move the fulcrum away from the apex; light forces move it closer to the apex.

In comparing tipping to bodily movement he noted that "clinically, with conventional fixed appliances, a greater force is usually required for bodily movement."

Sicher (1962) described the structure and function of the periodontal ligament. He admits that a one-rooted tooth can be observed to tip around an axis or fulcrum situated somewhere in the middle one-third of the root. He states, however, that since this claim rests on the observations of overloaded teeth whose suspensory ligaments have been damaged, the location of the fulcrum is still an open question. He adds that in multi-rooted teeth the location of the fulcrum has not even been attempted. This latter observation by Sicher, however, was not entirely 
accurate since Gantt (1960) had included in his study of tooth movement, a description of the center of tipping of the mandibular molar teeth during anchorage preparation with light forces. He had noted that the axis of tipping of the anchor tooth occurred more commonly in the apical third of the root than it did in the middle third of the root. His findings were confirmed by similar radiographic studies that followed by Stier (1961), Kemp (1962), Kravica (1963), and Follico (1964).

In addition to the studies reporting on the biologic aspects of tooth movement, other investigations were being conducted concurrently to determine the mechanics involved. A textbook on Dental Orthopedia by Case (1908) included a chapter entitled "Principles of Mechanics in the Movement of Teeth." From his discussion in this chapter of the different types of tooth movement; of the different kinds of levers; of the relations of power, stress, and movement; of the action line of forces; and of Newton's third law of motion, it is obvious that he was familiar with the true meaning of the word "mechanics" as described by physicists.

Fish (1917) summarized the trend of that time when he said:

The influence of technology on orthodontia demands your attention. Engineering methods, applied in the diagnosis and the treatment of malocclusion are producing results which mark the passing of empiricism in orthodontia. Where an art has stood, there is growing up a science.

It is significant that in that same year another article appeared in the International Journal of Orthodontia written by another consulting engineer. Hanau (1917) wrote "Orthodontic Mechanics; Dental Engineering" in which he defined such terms as force, resistance, velocity, work, 
energy and power, and such concepts as pressure on the root surface, projected root surface area, and volume of absorbed bone tissue.

Stanton (1928), although not an engineer himself, wrote about "Engineering in Orthodontic Diagnosis" and urged the dental and orthodontic societies and all dental schools to employ mechanical engineers to aid in teaching and explaining the engineering principles in dentistry and orthodontics. He further cited the great need for a textbook to be used in this field.

Drenker (1956) gave great impetus to the trend toward greater utilization of engineering mechanics in orthodontics. When he began his studies of dentistry and orthodontics he had already earned two degrees in Mechanica1 Engineering. He investigated and reported an analytical method for calculating the forces and torques associated with second order bends.

Weinstein and Haack (1959) stressed the idea that the study of theoretical mechanics, that is, the science of force action, is essential in orthodontics. In addition, they called attention to the need for an understanding of forces relative to the initiation of tooth movement. Particularly they emphasized the importance of the concept of equilibrium which provides a method by which the forces and couples acting in different planes and in different directions can be resolved and better understood. Such forces include not only those acting upon the crowns but also the reaction forces upon the roots.

Teasley, Penley and Morrison (1962) grouped the various means of resolving a force system into three categories: (1) the use of mathematical 
formulae, (2) physical measurements, or (3) by a combination of the two. They preferred to use physical measurements because it was the most practical method for the evaluation of orthodontic appliances.

In 1963, Weinstein, Haack, Morris, Snyder and Attaway, formulated a hypothesis which they referred to as "an equilibrium theory of tooth position." According to this hypothesis a tooth is considered to be a body in the state of rest or equilibrium, when the resultant of all the forces acting on the crown and those developed against the root is equal to zero. If the forces exerted upon the crown have a zero resultant, then the equilibrium of the tooth as a whole does not require the development of reactive forces upon the root. They conducted a clinical experiment which, they felt, established the validity of the theory.

Jarabak and Fizzel1 (1963) placed the greatest emphasis possible on the importance of biomechanics (or biophysics) of tooth movement by devoting four chapters of their textbook to this all-important aspect of orthodontics. They discussed in detail the fundamentals of analytical mechanics; applications of mechanics to orthodontic force systems; elementary strength of materials; and biophysical considerations of orthodontic forces. This comprehensive work embodies the most advanced concepts of the nature of orthodontic tooth movements and is, indeed, "a study of the biologic function, structure, and organization (of the teeth and the periodontium) in relation to and by the methods of physics."

B. Tooth Movement as a Function of Pressure

For centuries, physicists have known the difference between force 
and pressure. Force is the action of one body upon another - a push or a pull - measured in ounces, pounds, grams, or kilograms, etc. Pressure, on the other hand, is defined very precisely in physics as force per unit area and has the units of pounds per square inch or grams per square centimeter, etc. In orthodontics, however, the terms force and pressure have often been used rather loosely and inaccurately in the past. In fact, some orthodontists have considered the two terms to be synonymous and have used them interchangeably to describe the entity that brings about orthodontic tooth movements. Two examples of this are found in an article by Stuteville (1937) in which he reports that, according to A.M. Schwartz:

A force of $20 \mathrm{gm}$. per square centimeter of root surface will produce biologic tooth movement. He proves this by moving the premolars of dogs and showing that forces of $20 \mathrm{gm}$. or less produce a biologic tooth movement.

If Schwartz made the original error of calling a pressure a force, Stuteville did not recognize the inconsistency in terminology. Instead, he went on to describe the different tooth movements that he had achieved through the use of different forces.

According to Richmond (1933) "orthodontists have known only two pressures, viz., excessive and mild and have never been able to define either." This generalization again typifies the total lack of understanding that has persisted in the orthodontic literature with regard to the concept of pressure. Even in the present day, many orthodontists are still unaware of the fact that tooth movement is a function of compressive and tensile stresses developed within the periodontal ligament and not a 
function of force.

Angle (1907), in his textbook, speaks very accurately of areas of tension and compression in the periodontal space and states that as a result of pressure, the osteoclasts are stimulated to increase in number and activity. But since he does not define the term pressure nor make mention of the area of the root surfaces of the different teeth, one cannot be sure that he was referring to the physical concept of force per unit area.

As already noted, Schwarz (1932) attempted to relate tooth movement to pressure. From his orthodontic force experiment on one dog he concluded that the pressure resulting from the appliance should not exceed the capillary blood pressure. He also stated that the pressure of the tooth acting against the alveolus decreased as it approached the axis of rotation where the force was zero.

Renfroe (1951) was one of the first orthodontic theorists to suggest the idea that in tooth movement only a portion of the root surface is involved at any one time in resisting the movement of the tooth in the direction of the force. He alluded to the existence of an "effective root surface area" when he wrote: "The tooth with a purely round root when moved bodily, presents fifty percent of its periodontal membrane fibers to resist the movement and relaxes about the same number."

Storey and Smith (1952) investigated the effects that differential forces, developed from "light" and "heavy" springs, had on canines and on the anchor units. They found that the optimal magnitude of force for 
canine retraction ranged from 150 to 200 grams and for the movement of the anchor units (1st molar and 2nd premolar) the range was from 300 to 500 grams. One of the conclusions they drew from their study was that:

Undoubtedly it is not the force that is exerted on the tooth that is significant, but rather the pressure (i.e., force per unit area) which is exerted at the interfaces between tooth, periodontal membrane and bone. It is this pressure and its distribution over the surface of the root that will be difficult to estimate for various appliances and this could limit their proper design.

Thus, they were the first investigators to focus attention on the a11-important concept of tooth movement being a function of pressure (in the true physical sense) rather than simply a function of force.

Shroff (1953) claimed the periodontal membrane to be composed of a loose fibrous connective tissue imbedded in a viscous tissue fluid. He further stated that the forces of occlusion were transmitted to the bone in two modes; first, as pressure due to the fluid content of the periodontal membrane and, second, as tension due to the collagen fibers.

It had been stated by Boyle (1949) that these pressure effects are rapidly dissipated due to the expulsion of fluid by way of the adjacent marrow spaces and vascular channels and that the mechanism is similar to a "hydraulic and snubber absorber system."

Shroff, however, pointed out that it was erroneous to regard periodontal tissue fluid as a "simple fluid subservient to the ordinary laws of hydrostatics." He maintained that this fluid was very viscous and, moreover, was confined by a complex meshwork of fine and coarse collagenous fibers and cells. Applied pressure was not dissipated as rapid1y as 
supposed and such pressure would not be equally transmitted in all directions as with simple liquids.

Much of what is known and understood about the application of force in orthodontics has been derived through empirical methods. Although treatment procedures based on empirically designed appliances have resulted in unquestionable success, this procedure has contributed little to the basic understanding of exactly how a given appliance operates, what its variables are, how pressure is developed and distributed to the periodontal ligament, and other pertinent information. The empirical method has thus limited the complete analysis of appliances to the simplest forms of force application such as forces exerted by coil springs, elastics, and other unidirectional appliances. This situation has presented itself as a result of the educational preparation of the orthodontist which has, in the past, been limited in its scope relative to physics, engineering and mathematics.

MacEwan (1954) illustrates this fact in his published article describing his treatment of a typical distocclusion case. He observed empirically that through the use of an upper "buccal bar", light elastics, and a rigid lower lingual arch as anchorage, the mandibular (anchor) teeth remained undisturbed regardless of the length of time that intermaxillary elastics were being used. He explains that:

This is possible because the amounts of force used are kept below the stability limit, which is about $7 \mathrm{gm}$. per square centimeter of root surface (or, more precisely, per square centimeter of alveolar lamina dura) if the periodontium is physiologically normal. 
Here we find reference to a value for the subliminal pressure against the alveolar wa11, below which a11 movement ceases.

How MacEwan arrived at the value of $7 \mathrm{gm} / \mathrm{cm}^{2}$ as the "minimal" pressure which causes no movement was later revealed by Stoller (1964). In a paper read before the American Association of Orthodontists, he stated that MacEwan had calculated the total root surface area of all the lower teeth to be $12 \mathrm{~cm}^{2}$ :

\begin{tabular}{cl} 
Area of Root Surfaces & \\
\hline Lower central incisor & $=1.0 \mathrm{~cm}^{2}$ \\
" lateral incisor & $=1.0 "$ \\
" canine & $=2.5 "$ \\
" 1st premolar & $=2.0 "$ \\
" 2nd premolar & $=2.0 "$ \\
" lst molar & $=3.5 "$ \\
Total Area (one side) & $=12.0 "$
\end{tabular}

MacEwan then measured the force exerted by the intermaxillary elastic on each side to be 3 ounces, or 84 grams. This total force of 84 gm. was distributed over six mandibular teeth on each side having a combined total root surface area of $12 \mathrm{~cm}^{2}$. Thus,

Pressure exerted by each tooth $=\frac{84 \mathrm{gm}}{12 \mathrm{~cm}^{2}}=7 \mathrm{gm} / \mathrm{cm}^{2}$.

It is obvious, of course, that this is a gross over-simplification of the complex concept of subliminal pressure because: (1) the force measured was in an oblique direction and thus did not represent the horizontal component which was tending to drive the anchor teeth forward; (2) the root surface areas were only roughly estimated rather than measured accurately; and (3) total root surface area was used in the calculation of pressure, whereas only a portion of each root surface was actually 
involved in resisting the movement of these teeth in a forward direction.

Burstone et a1. (1961) very adequately described the interrelationship of force and pressure in orthodontic tooth movements when they stated that :

Force in orthodontics is useful only insofar as it initiates a desirable tissue response. It seems reasonable to assume that this response is a function of the pressure distribution in the periodontal membrane when a force is applied to the coronal portion of the tooth......Force is distributed throughout the membrane in a manner which depends upon the root (length, diameter and contour), the nature of the periodontal membrane and the site of force application.

Sicher (1962) described the function of the periodontal ligament as being similar to the protective and limiting action of other ligaments in the body. He claimed that masticatory movements bring pressure to bear on the periodontal ligaments of the teeth which leads to compression of the tissues. The veins are temporarily emptied and thus injurious pressures on the cellular elements are prevented. This protective function is augmented by the fact that the suspensory fibers on the tension side limit the movement of the teeth during mastication.

It is noteworthy that Sicher pointed out that one should employ the proper terminology when speaking of the movement of a tooth within its alveolus. In tipping, for example, he states that one should describe areas of tension and compression (rather than of tension and pressure). This is certainly more consistent with the actual definitions of these terms as used in physics. Tension and compression are both types of stresses that can develop within a material, while pressure is the amount 
of force acting over unit area.

Jarabak and Fizzel1 (1963) applied logic, analytical thinking, physics and mathematical disciplines to their evaluation of the fundamentals of tooth movement. They were led to the conclusion that "the first requirement is to accept the idea that root pressure is the important factor in determining tooth movement instead of the force applied to the crown of the tooth." They reasoned that since experience has shown that a certain magnitude of force is needed to start a tooth moving and less force is required to keep it moving, there must be three important values of root pressure: $\mathrm{P}_{\mathrm{u}}=$ Supramaximal pressure at which undermining resorption occurs, $\mathrm{P}_{\mathrm{m}}=$ Average root pressure needed to start translation of a tooth, and $\mathbf{P}_{\mathbf{i}}=$ Subliminal pressure below which all movement ceases. Although the values of these pressures are still unknown at this time, they do not cease to be real values with units of grams per square millimeter. With this concept as a basis, they were able to express mathematically the conditions that must be met for tooth movements to be possible at all.

C. Use of Models to Study Tooth Movement

In the past, various investigators have used models of teeth to illustrate more effectively or to study the manner in which a tooth moves within the limits of its alveolus and, orthodontically, by means of resorption of bone. In general, two types of models have been employed; physical models and mathematical models.

Case (1908) was one of the earliest writers who used a model to 
illustrate simple tipping. He drove a stake into the ground to a depth of approximately two-thirds its length and then applied to the exposed end, a force that was perpendicular to the stake (Figure 2). He demonstrated that the exposed end (the crown) moved in the direction of the force while the tip of the buried end (the root apex) moved in the opposite direction. He noted that tipping occurred about an axis somewhere near the middle of the buried portion or root.

Schwarz (1928) performed basically the same experiment but he used a match stick which he placed into soft plaster (Figure 3). Before the plaster had a chance to set, he applied a perpendicular force to the end of the stick. The results were the same as Case had found, but he could demonstrate it more effectively with the hardened plaster.

But Schwarz went a step further. He assigned values to the dimensions of the model and to the applied force, and from this mathematical model, determined the precise location of the axis of rotation in terms of $h$ and $w$ (Figure 4). He then observed that it made little difference where the force was applied on the crown portion. The center of rotation did not vary significantly as $h$ diminished down to zero, provided the direction of force remained the same.

Bauer and Lang (1928) reported that Schwarz had made a fundamental error in all his mathematical calculations since he did not take into account the fact that the roots of teeth are not of uniform breath, but rather more conical in shape. Thus, they analyzed a mathematical model (Figure 5) which incorporated three basic assumptions: 


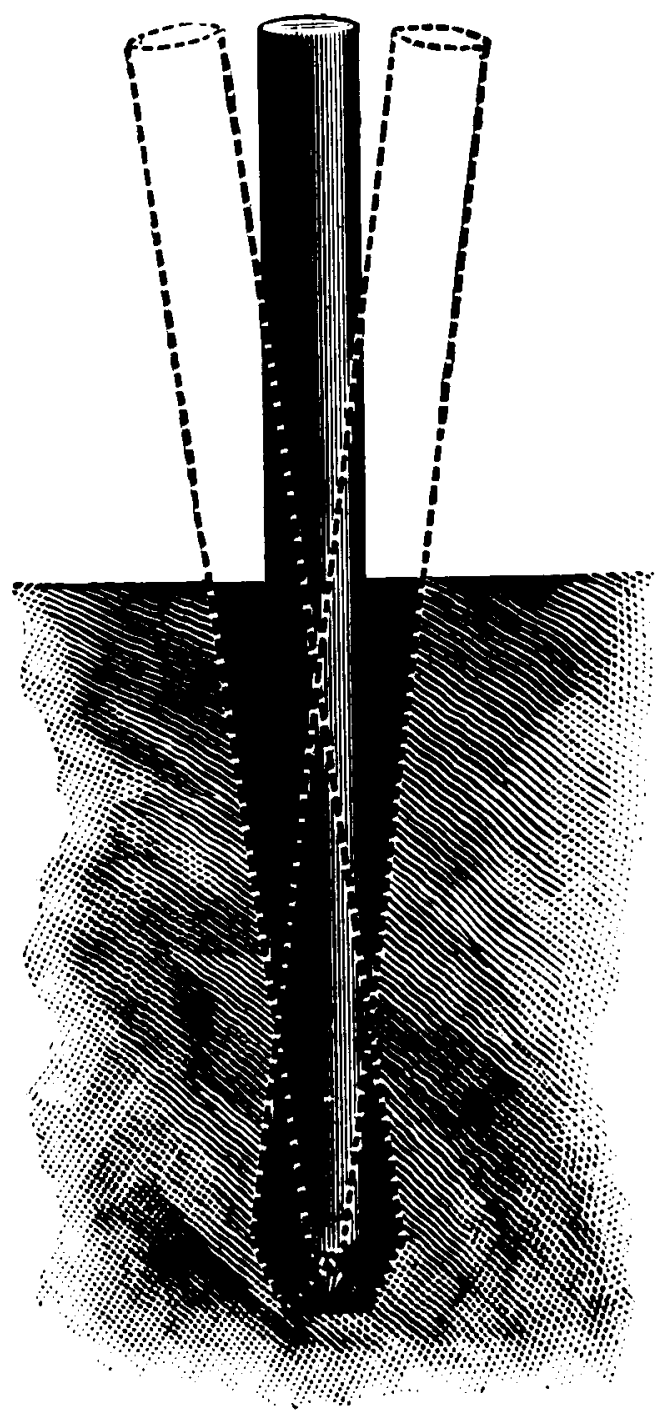

FIGURE 2

POST IN THE GROUND EXPERIMENT BY CASE 


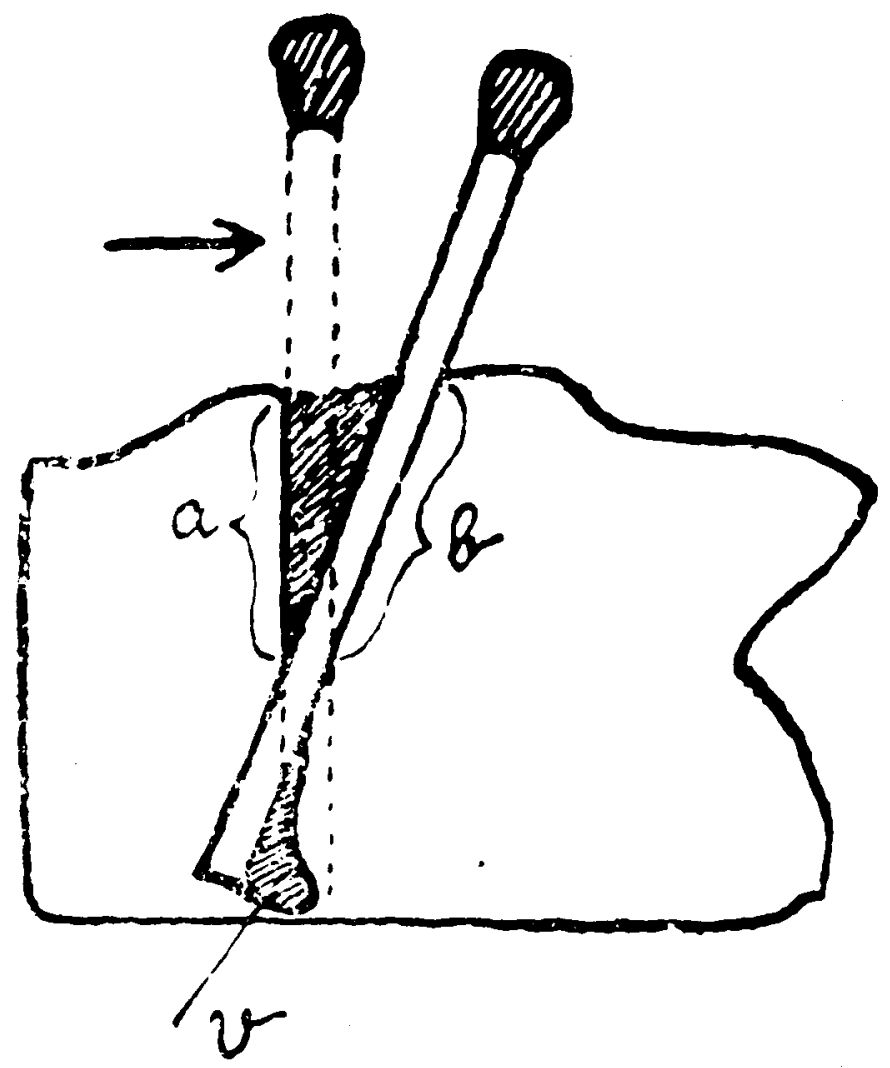

FIGURE 3 


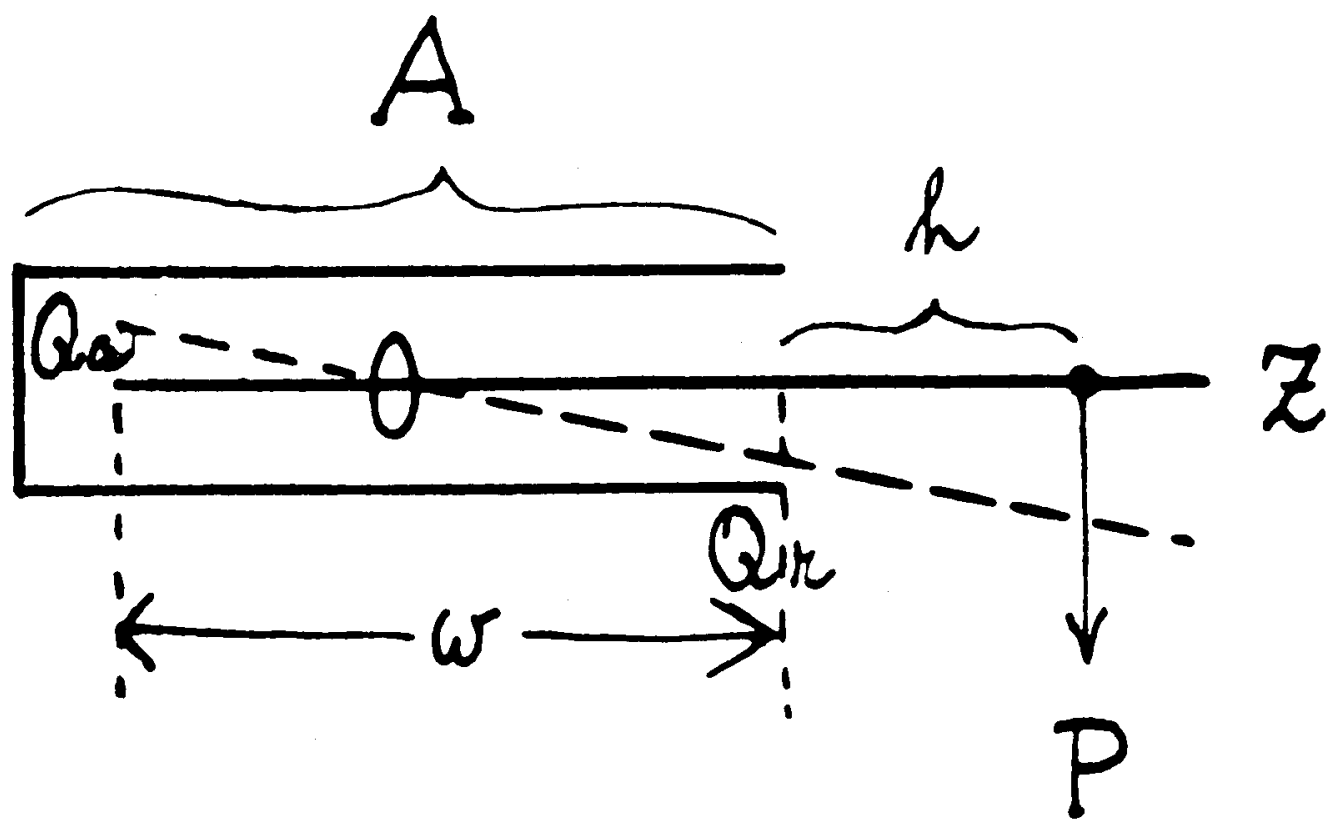

\author{
FIGURE 4
}

MATHEMATICAL MODEL USED BY SCHWARZ 


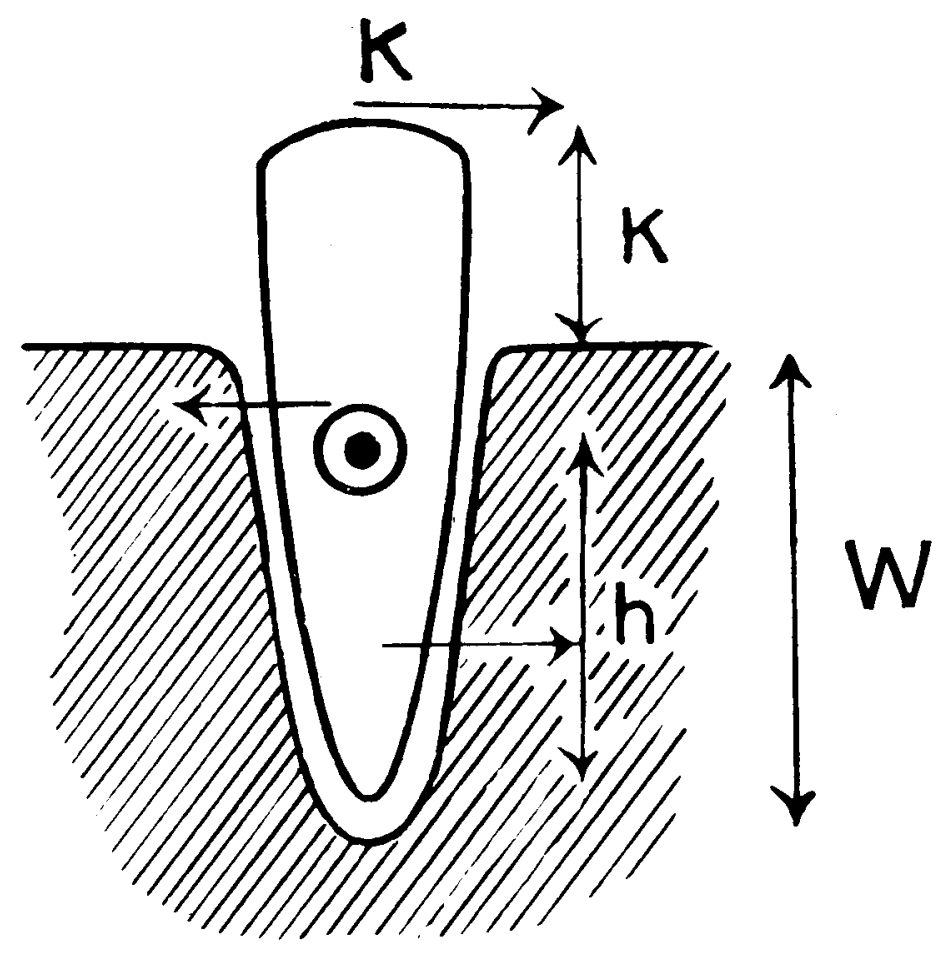

FIGURE 5

MATHEMATICAL MODEL USED BY BAUER AND LANG 
1st, that the root is cone-shaped,

2nd, that the root is two-thirds the length of the tooth, and

3rd, that the force is applied at the extreme end of the crown perpendicular to the long axis of the tooth.

Their equation, in terms of $\mathrm{h}$ and $\mathrm{w}$, for the location of the center of rotation differed from that of Schwarz, but one reason was that they labeled the dimensions on their model differently.

In 1930 , Fickel confirmed the mathematical calculations of Bauer and Lang and he compared them with those of Schwarz. To achieve an accurate comparison he made a model like the one used by Schwarz and another one like the one used by Bauer and Lang, but he made them of identical dimensions and similar in all respects except that one was cylindrical (Figure 6A) and the other one cone-shaped (Figure 6B). In addition, he constructed still another mathematical model of his own in which the root was assumed to be a paraboloid curve. After determining the mathematics related to the loading of his own model, he concluded that Bauer and Lang's calculations were closer to his equation for the location of the center of rotation and that Schwarz's calculations were incorrect. Nevertheless, he proceeded with an experiment (similar to Schwarz's match stick experiment) in which he embedded a post in clay and pushed on it with a force perpendicular to the post. The post moved (tipped) in the clay and left an impression or void which he filled with Mellott's metal after he had removed the post. On the resulting metal pieces (Figure 7) he measured the distance from the lower end to the area of minimum width 

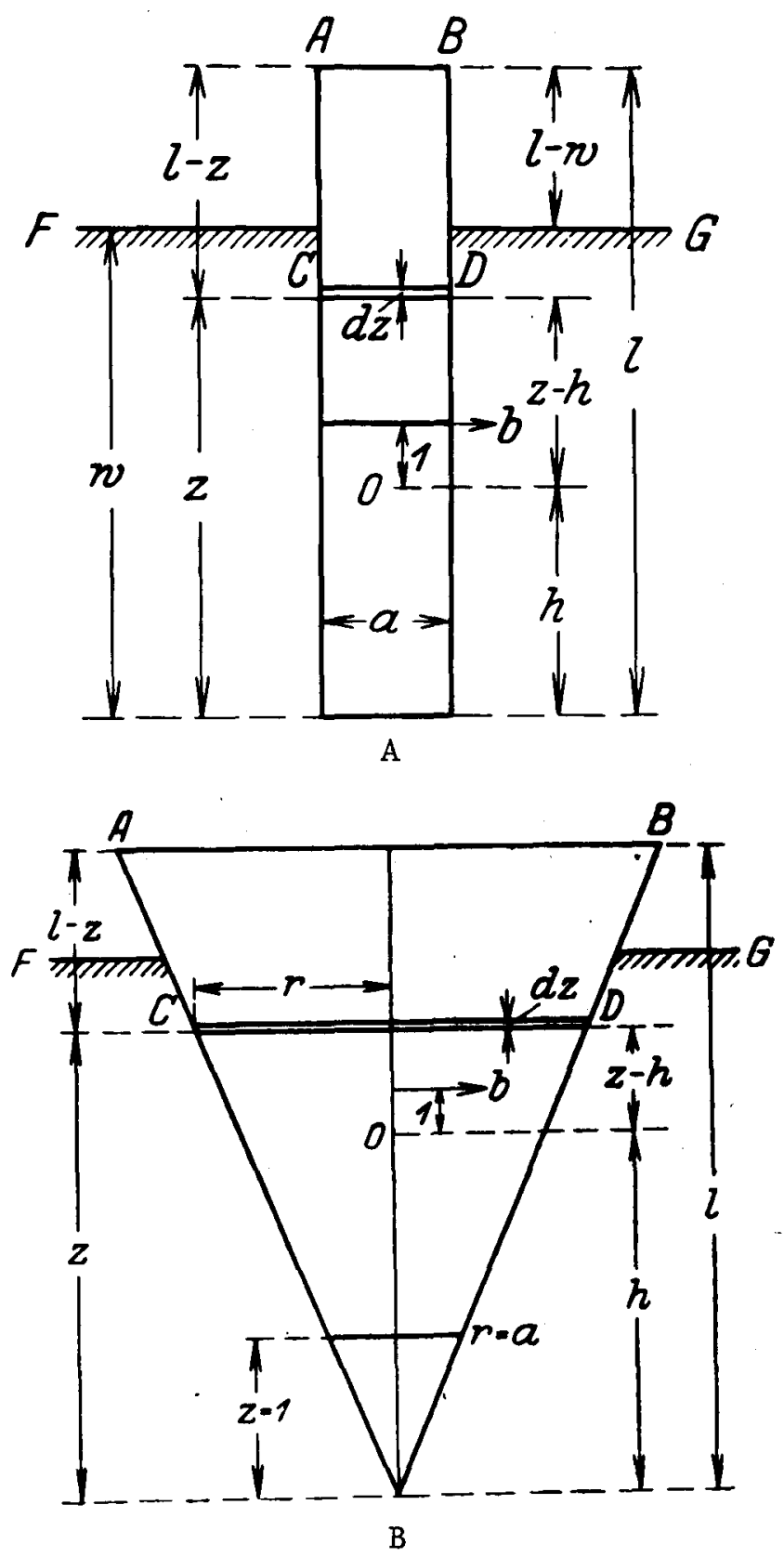

FIGURE 6 


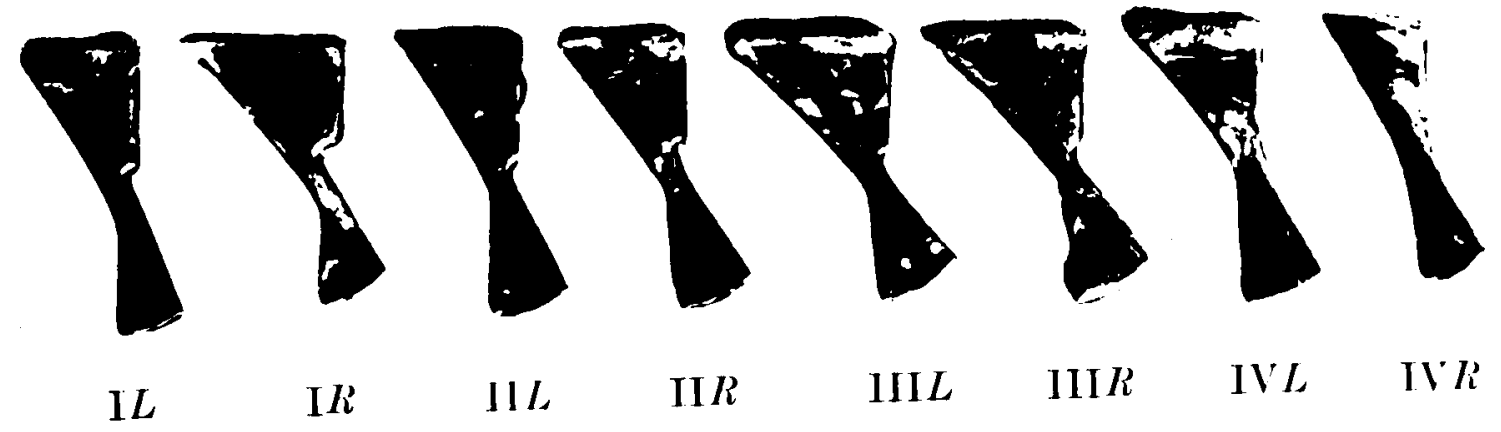

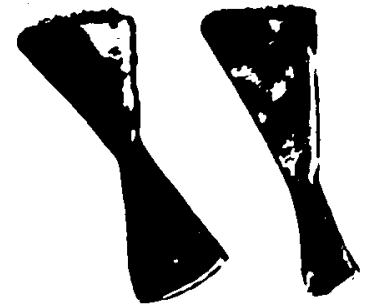

$\checkmark L$

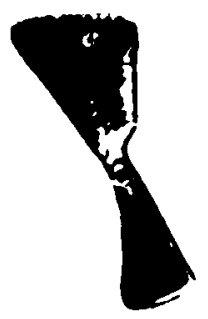

VIL

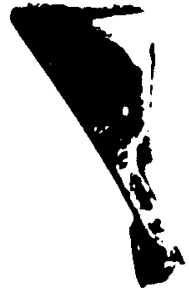

$\mathrm{VI} R$

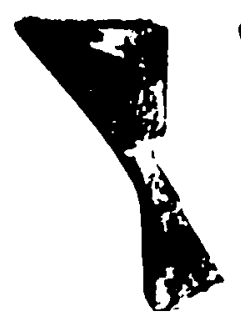

VII $L$

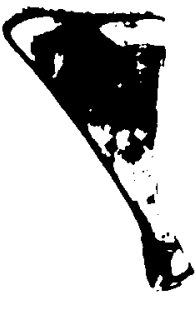

VII $l$

\section{FIGURE 7}

METAL PIECES FROM EXPERIMENT BY FICKEL 
(center of rotation). After repeated trials he concluded that the location of the center of rotation was a function of the speed with which the post had been moved through the clay.

Synge (1933), in an article describing the theory of an incompressible periodontal membrane, pointed out that the question of how a tooth turns when it is subjected to occlusal forces could be studied either by means of an enlarged model of a tooth made of wood with the periodontal area filled with an incompressible, elastic substance such as rubber, or by working out mathematically the behavior of such a model without actually constructing it. Since he was Professor of Applied Mathematics at the University of Toronto, he preferred to use the theoretical mathematical method because he felt it would be more "powerful" provided that proper attention were given to the limitations of mathematics in drawing conclusions. He readily admitted that in using his mathematical model certain "simplifying assumptions" had to be introduced in order to make interpretation of the results possible.

In another article (The Tightness of the Teeth, etc.) published in the same year (1933), Synge stated the problem as follows:

An elastic membrane fills the space between, and is attached to, two rigid bodies, the tooth and the socket, of which the former is subjected to assigned forces, while the latter is held fixed. We assign to the membrane the following properties:

(i) infinitesimal, but not necessarily uniform, thickness;

(ii) homogeneity;

(iii) isotropy;

(iv) incompressibility;

(v) finite rigidity 
and we shall suppose that the free edge, or margin, of the membrane is subject to atmospheric pressure. The problem is to investigate the equilibrium of the system, particularly with regard to the displacement of the tooth and the strain and stress in the membrane.

The hypothesis that the membrane was incompressible had been expounded by Gysi (1930). It was based on the assumption that 80 percent of the tissues of the human body is water, which, for all practical purposes, is incompressible. The periodontal membrane - also being a tissue largely composed of water - was believed to experience no change in volume except "when blood in the blood-vessels is squeezed back into the bony structure or into the adjacent mucous membranes by a prolonged pressure."

The theorem developed by Synge showed that the displacement under a finite force is proportional to the cube of the thickness of the incompressible, elastic membrane of finite rigidity. For a conical model with a membrane of uniform thickness several other facts were salient:

1st, the pressure in the membrane at the gingival margin was always equal to atmospheric pressure.

2nd, under an axial load the pressure decreased from a maximum at the apex to atmospheric at the margin.

3rd, under a transverse load the pressure was atmospheric at the apex, the margin, and at an intermediate point near the center of rotation. There were two points each of maximum and minimum pressure located between the center of rotation and the apex, and between the center of rotation and the gingival margin. 
$4 \mathrm{th}$, the distance between the center of rotation and the root apex (along the long axis of the tooth) was determined only by the point of intersection of the action line of the force with this axis. The distance between the center of rotation and the long axis of the tooth was determined by the above point (of intersection) and also by the direction of the force. For a transverse force at the incisal edge of the coneshaped model the center of rotation was on the long axis about threefourths of the root length from the apex.

Zak (1935) introduced a unique, new method of photo-elastic analysis of the mechanics of orthodontic tooth movements. He used metal models of teeth, 2 or $5 \mathrm{~mm}$. thick, embedded in corresponding alveoli of celluloid as well as celluloid teeth of the same size embedded in metal alveoli (Figure 8). By applying known, single forces and combinations of forces to the crowns of the mode1 teeth, he said that he was able to reproduce movements that could be brought about by a removable appliance, by Angle's pin and tube appliance, the ribbon arch, the edge-wise arch, McCoy's open tube appliance and Case's appliance. The mechanical strains produced in the celluloid tooth or alveolus were visible because of the double refraction of light through the celluloid. Hence, qualitative tensions arising in the tooth root and its surrounding alveolus were studied and described. By this optical method, he also determined the center of rotation in tipping as well as the conditions that lead to tipping or translation (bodily movement) of teeth.

Hay (1939) concurred with the mathematical calculations that had 


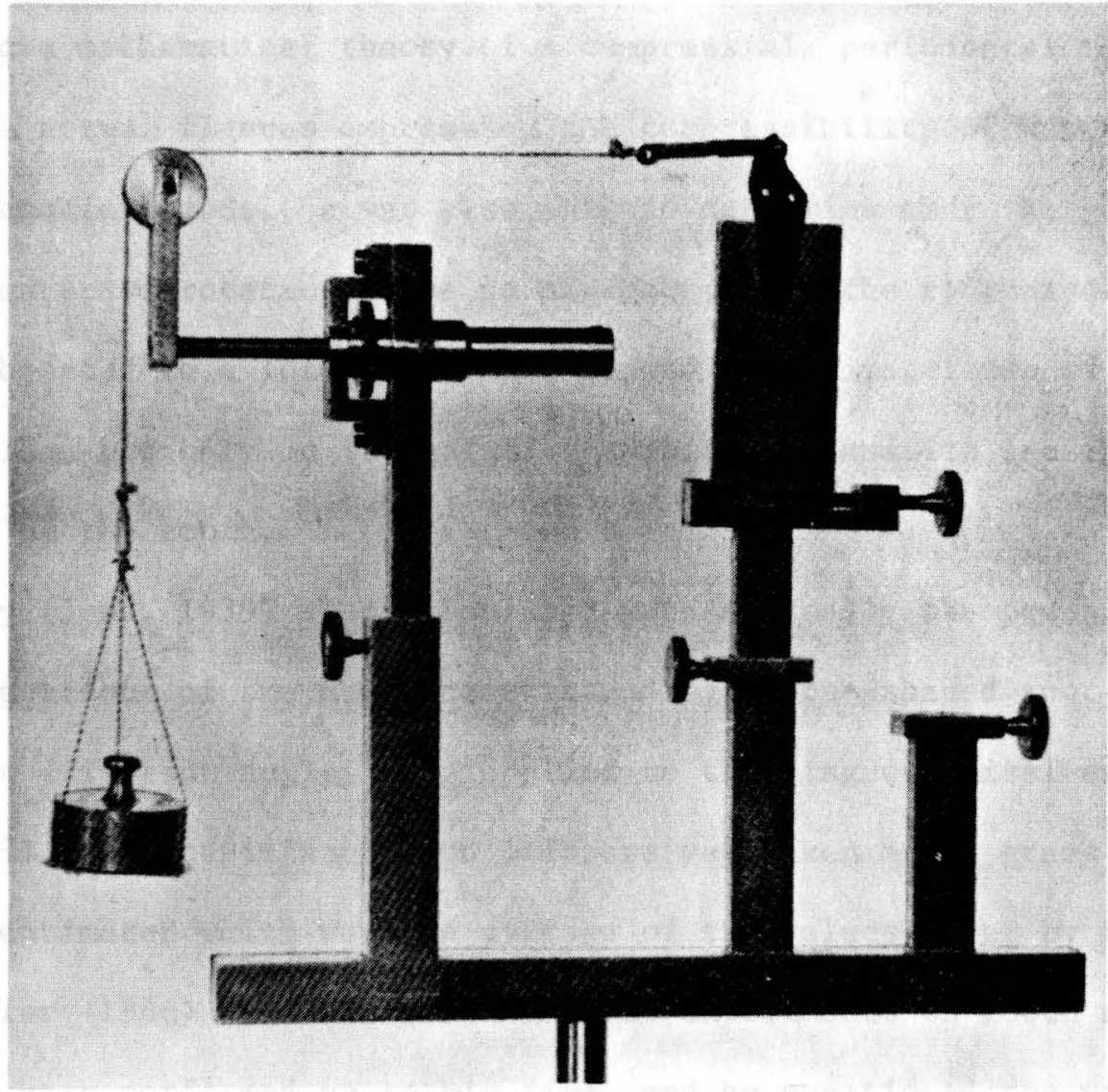

FIGURE 8

APPARATUS USED BY ZAK FOR PHOTO-ELASTIC 
been advanced by Synge regarding the periodontal membrane. But he considered the possibility of a compressible membrane since he felt that the compressibility of water is not small enough to be neglected. Thus, he developed a mathematical theory of a compressible periodontal membrane using the actual figures expressing the compressibility of water. From his mathematical model he was also able to determine that the position of the center of rotation ("the point about which the tooth rotates when it is subjected to a load") does not depend on the magnitude of the applied load but only on its point of application and its inclination to the axis of the tooth.

Hay (June, 1939) also calculated mathematically the pressures in various portions of the membrane surrounding a cone-shaped root when forces at different angles were applied to the linguo-incisal edge (Figure 9). The capillary blood pressure was taken at 23 grams per square centimeter which was the average of the values given by Schwarz. Adler (1946) reviewed all the model experiments on tooth movement that had been performed up to his time, and he questioned the validity of the basic assumptions made by previous authors in the development of their theories. For example, he did not believe that the periodontal membrane was homogeneous as most authors had assumed. He postulated that there must be some difference between the central and apical periodontal membrane which would account for the greater degree of root resorption at the apex. Thus, he felt that a model showing tooth movement should be such that it would also allow tipping about the apex (as Angle 


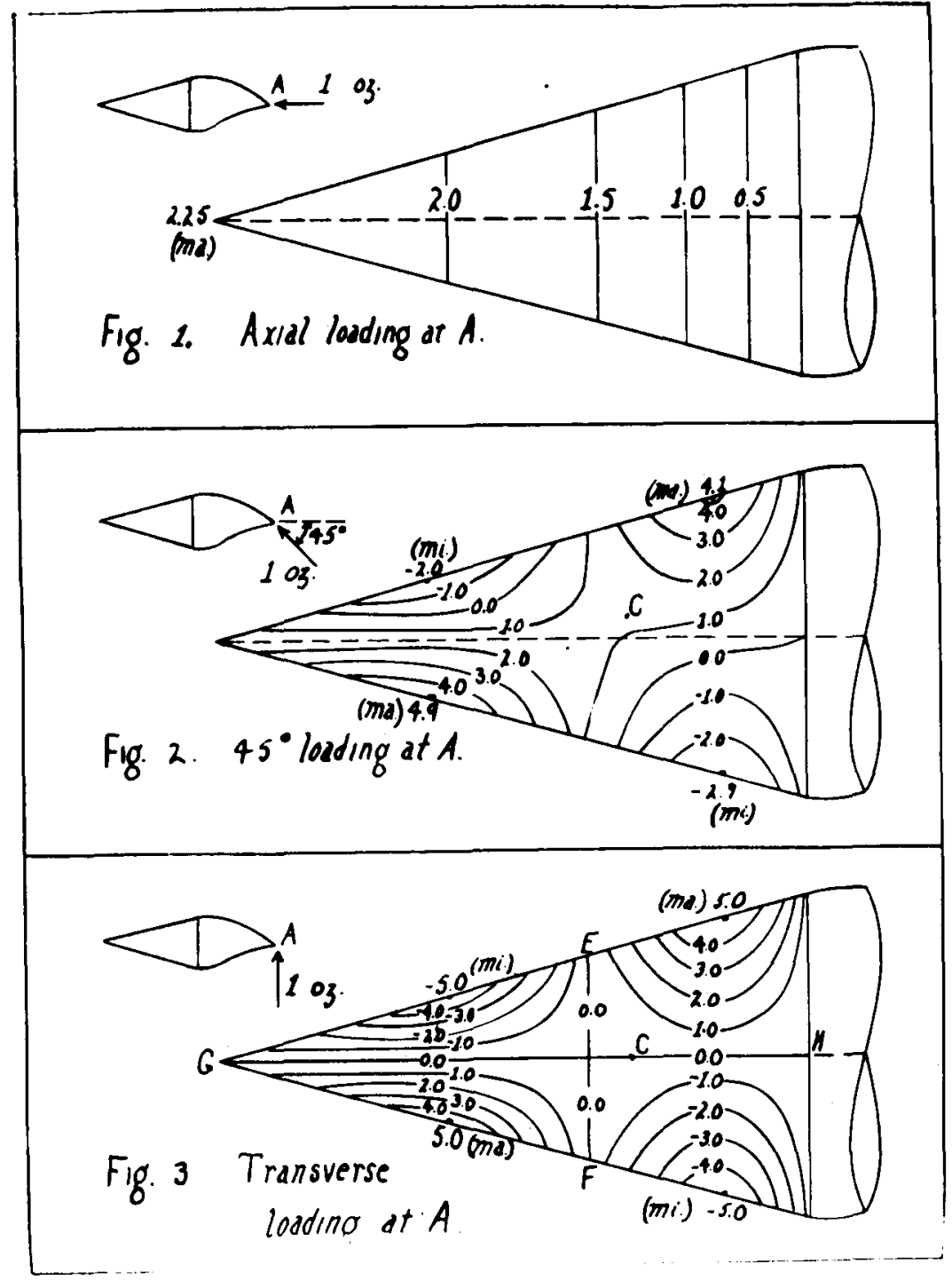

FIGURE 9

DIAGRAMS DEVELOPED BY HAY ILLUSTRATING PRESSURES ON A MEMBRANE SURROUNDING A CONE-SHAPED ROOT 
and Oppenheim had described).

Adler also presented arguments intended to show that the strong distinction made by some authors (notably Schwarz) between tooth movements within the socket and those due to tissue changes was untenable. In other words, he questioned whether it was logical and accurate to consider two types of motion of a tooth: primary movement - within the alveolus, and secondary movement - after change in the wa1ls of the alveolus.

Shwalb and Rechter (1950) constructed a transparent Bakelite model of a bone socket and an acrylic tooth model was fitted and adapted to it. Several types of orthodontic forces were applied to the crown portion of the model tooth and the transparent socket was viewed through a polariscope. The internal stresses set up in the Bakelite model were photographed and the patterns of stress were related to the magnitude and direction of the applied forces. They then discussed the correlation of orthodontic principles with experimental results.

Burstone (1962) stated that there are three levels of observation that can be used to describe the response of a tooth to forces: clinical, cellular, and stress-strain. He believed that perhaps the most important and least understood is the stress-strain level of activity in the periodontal membrane. He emphasized the importance of models of teeth as good research tools when he said:

Since at this time it is not possible to place strain gauges in the periodontal membrane to measure stress distributions, our knowledge of stress phenomena must 
depend on another approach. A mathematical model of the tooth and surrounding structures can be constructed based on certain assumptions. From these mathematical models theoretical stress levels can be calculated if the forces applied to the teeth are known.

Burstone also recognized the fact that to describe tooth movements specifically and accurately a method would have to be evolved whereby the center of rotation could be determined and described relative to three mutually perpendicular planes. For purposes of simplification, however, he limited his discussion to two-dimensional representations of teeth thus considering only one plane of space. The assumptions that he made were that stress distributions in the periodontal ligament were uniform for pure translation and uniformly varying for pure rotation (tipping). Since he also postulated that a linear stress-strain relationship exists, he was able to predict mathematically the force system required for various centers of rotation.

Haack and Weinstein (1963) used a two-dimensiona1, wooden mode1 of a maxillary central incisor which had elastic foam sponge in the space between the root and alveolar process, to illustrate the ways in which the application of forces to the crown of a tooth introduces a distribution of forces in the periodontal ligament (Figure 10). They believed that "it is the nature of this distribution which determines the pattern of bone resorption and apposition and thus the whole complex geometry of tooth movement." They were convinced that it was of great importance to the practitioner of orthodontics and the graduate student to have a clear understanding of this phenomenon and to be able to demonstrate it 


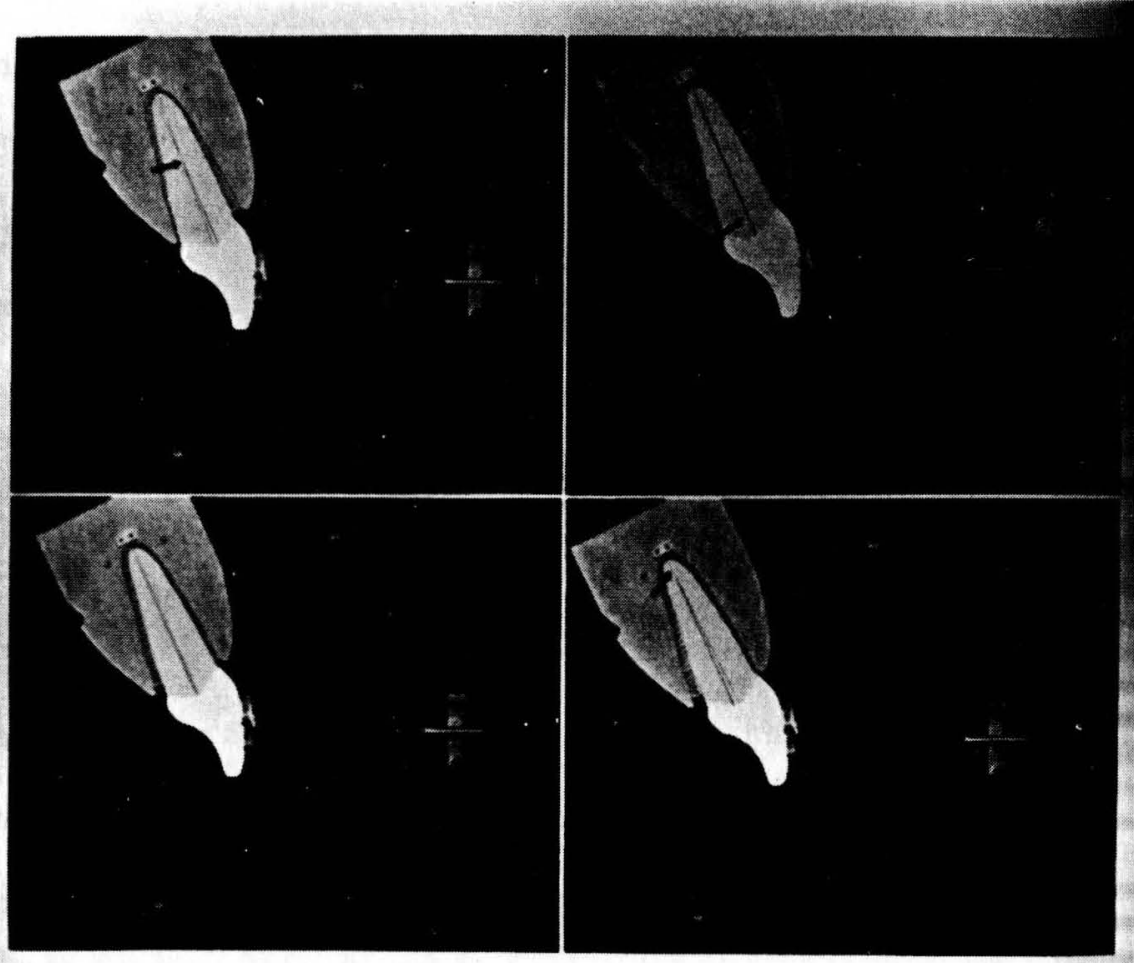

Fig. 7. Above left: Application of a pure moment. Center of ratation at point slightly less than half of root length from opex. Above right: Pure moment plus a light force sufficient to shift center of rotation to alveolar crest level. Below left: Moment the same, but increased force. Center of rotation shifted to infinity. Below right: Moment same, but further increase of force. Center of rotation shifted to apex 
qualitatively. As far as a mathematical analysis of the force distribution or a quantitative solution to the problem is concerned, they felt that it was possible "only if rather severe simplifying assumptions are imposed" which often made their clinical application impractical.

Jarabak and Fizzel1 (1963) employed mathematical models as an adjunct in their description of the biophysics of orthodontic forces. In their description of translation of a tooth, a parabola was used to represent the contour of the root of a mandibular right canine (Figure 11). With this geometric analog of the root of a tooth, they were able to employ integral calculus in order to determine: (1) the projected area of the root (or parabolic curve, closed at the top), (2) the first moment of area of the figure around the $\mathrm{X}$-axis, and (3) the average moment arm (by dividing the first moment of area by the area of the parabola). With a knowledge of these values it was possible to solve for the centroid of area. By means of this mathematical model the concept of "effective root surface area" was developed and the theory of "effective root pressure" advanced.

Dempster and Duddles (1964) employed enlarged models of a tooth and its socket made of composition board representing either buccolingual or mesiodistal cross-sections of a lower molar (Figure 12). Through the application of a coronal force acting at a definite angle, the tooth moved and made contact with the socket. Force gauges were attached to the model tooth at the points of tooth-socket contact and forces perpendicular to the surface were measured at these points. Free-body diagrams 

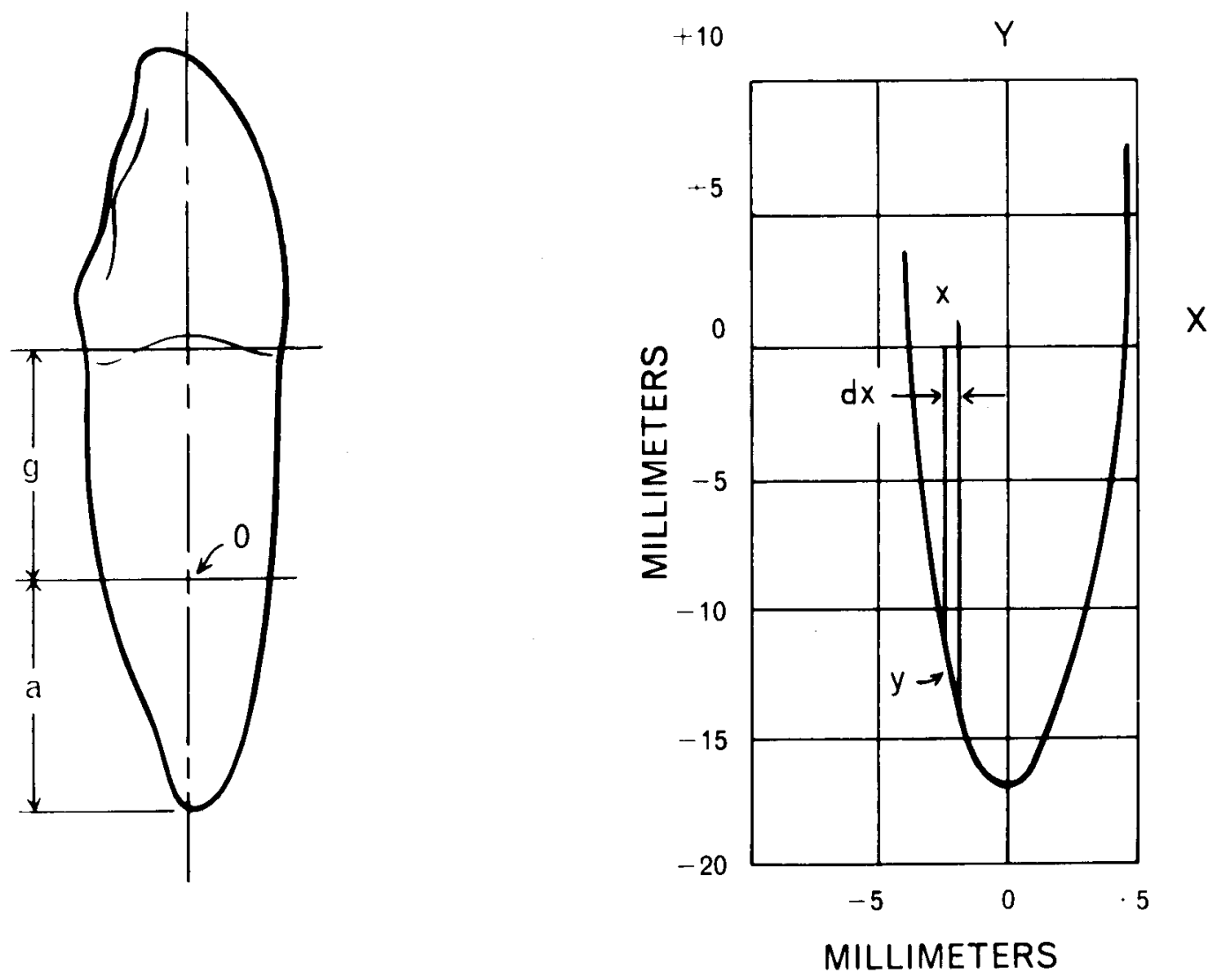

FIGURE 11

MATHEMATICAL MODEL USED BY JARABAK AND FIZZELI 


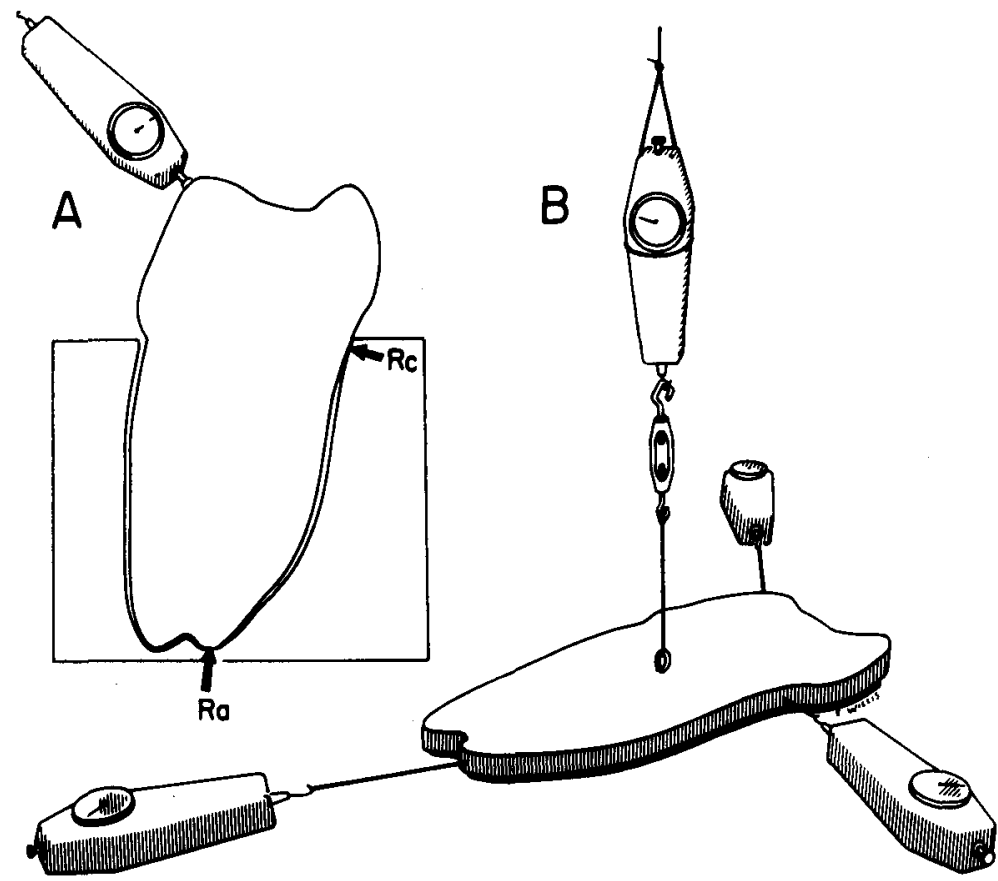

Fig. 3 - A:Chipboard model of tooth and socket: compression force gauge pushes tooth into contacts with socket model. B:Tooth model suspended above penciled outline on work table with tension force gouges attached at points normal to tooth profile. All forces ore in balance 
of each equilibrium situation included the magnitude and direction of each force and its point of application. For a given set of conditions (when the forces were in equilibrium), the gauges measured forces comparable to those involved in the statics of the tooth.

Isaacson and Ledley (1964) found fundamental errors in the study reported by Dempster and Duddles. They pointed out that, whereas Dempster and Duddles completely ignored consideration of shear forces, "the periodontal membrane exerts reactive shear forces and these forces may be the most important aspects of a force analysis for a tooth." They also took issue with the assumption made by Dempster and Duddles that the reactive forces must be normal to the surface on which they act. The authors felt that this assumption would be valid if the points of contact (between tooth and socket) were frictionless, which is not the case with the real tooth or even with the chipboard model. Four of the illustrations used by Dempster and Duddles to depict the balance of moments in a situation of equilibrium were found to be totally incorrect. Other figures were only "slightly unbalanced." Finally, the authors felt that Dempster and Duddles had not considered functional forces correctly and that it was inaccurate to describe "six degrees of freedom of tooth motion" because a tooth is constrained by the periodontal membrane and attached "very well" to the bony jaw. They stated that six degrees of freedom would exist only for an already extracted tooth, as for any unattached three-dimensional body. 
CHAPTER IV

\section{PROCEDURE}

A. Selection of the Model Tooth

A three-dimensional, enlarged, anatomical model of a lower left canine was used in this investigation (Figure 13). This model tooth was selected from the new series of twenty-eight normal teeth and nine variations by Dr. P.A. Lindstrom of Stockholm, Sweden. These models of human teeth are made by the Denoyer-Geppert Company, Chicago, Illinois. They are enlarged so that the height of each model (along the long axis) is ten times that of an average sized tooth. They are cast in an unbreakable 1ight-weight plastic and are hollow.

The lower left canine was selected for three reasons: (1) it was felt that a single-rooted tooth would make the measurements of tipping and translation more uniform and easier to interpret, (2) it is a tooth that frequently requires tipping distally (or translation) in actual clinical situations and, thus, the information derived from this study would be directly useful, and (3) it would be upright when placed in a plastic alveolus and set on a platform; thus, it could very conveniently be made to have the same spatial relation on a table as this tooth has in the mouth.

Although the plastic model tooth was comparatively rigid and 


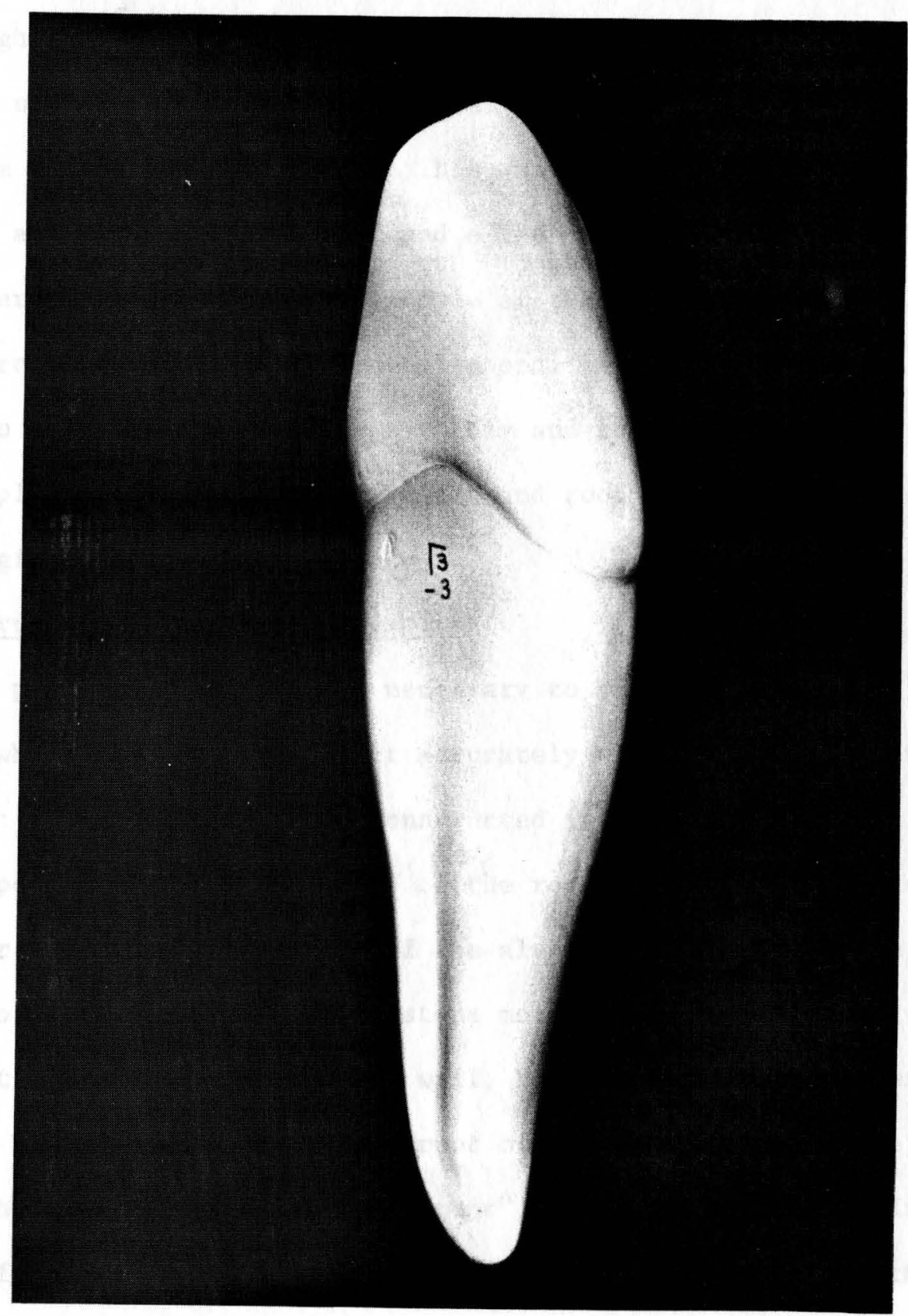

FIGURE 13

PLASTIC MODEL OF A LOWER LEFT CANINE 
inflexible, the fact that it was hollow made it possible to distort the tooth slightly by applying a compressive force on it. To eliminate this potential source of error, the tooth was made into a solid mass by filling the inside with Plaster of Paris. This was accomplished by drilling a $1 / 4^{\prime \prime}$ hole at the apex of the root and a $1 / 8^{\prime \prime}$ hole at the tip of the crown. The plaster was poured through the hole at the apex and air was allowed to escape through the hole at the opposite end. A vibrator was used in the process to make the plaster flow smoothly and to avoid trapping air. When the plaster had set, the crown tip and root apex were recontoured to their original shapes.

\section{B. Construction of the Model Alveolus}

Two preliminary steps were necessary to construct the plastic alveolus into which the tooth would fit accurately with its rubber "periodontal ligament": (1) a mold had to be constructed into which a liquid plastic could be poured; (2) a stone model of the root of the tooth had to be made to provide the inside shape of the alveolus, which was to be cast in liquid plastic. Since the latter stone model would be used to give us not only the shape of the alveolar wall, but also the inside dimensions, it had to be wider than the actual root of the model tooth by the thickness of the rubber "periodontal ligament" that was to be used. In this way, the finished alveolus would accomodate exactly the root of the model tooth together with the uniform thickness of rubber "periodontal ligament". The mold that was used for the pouring of the liquid plastic was a wooden box whose inside dimensions were: $3.5^{\prime \prime} \times 3.5^{\prime \prime} \times 8.0^{\prime \prime}$ (Figure 14). 


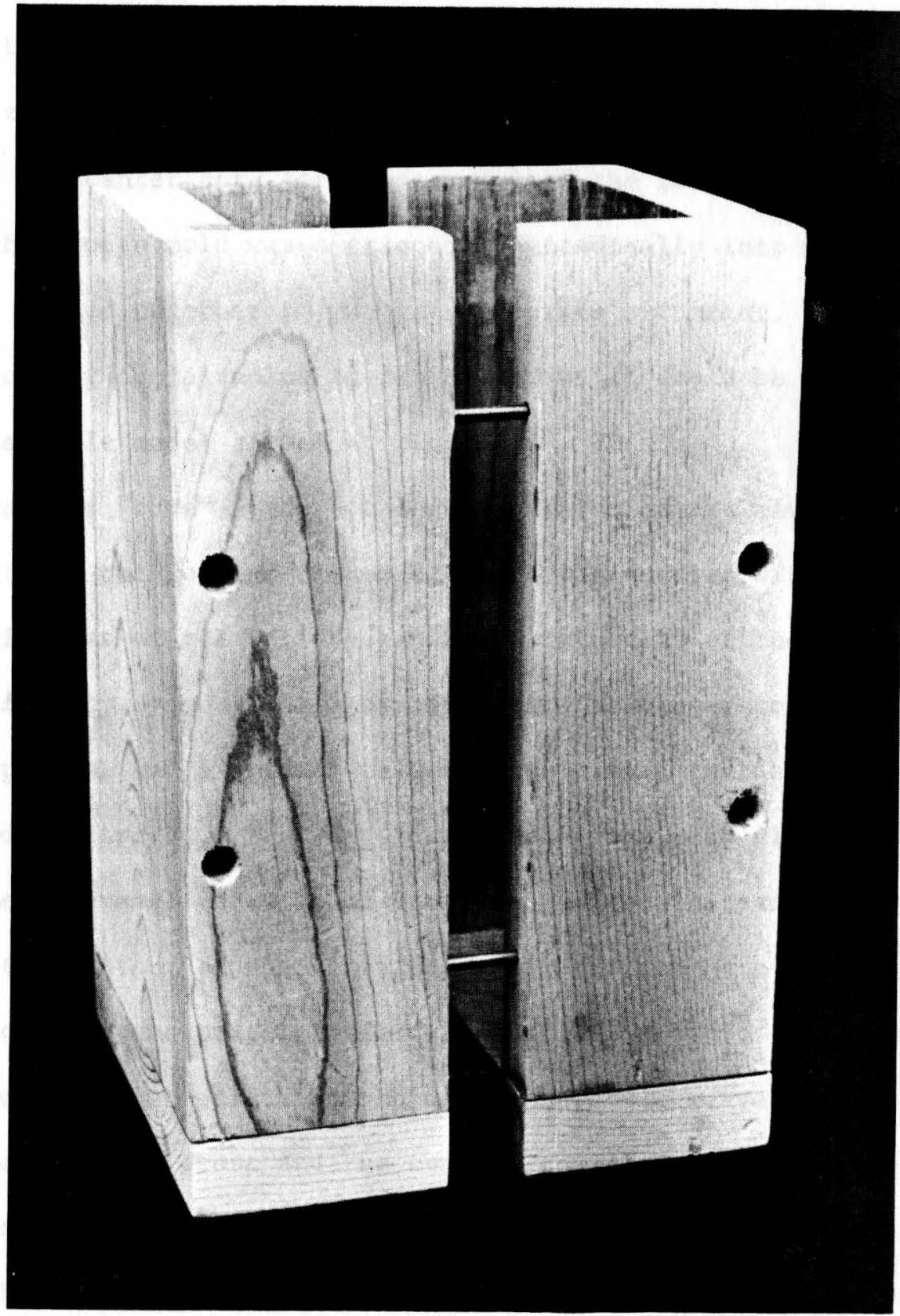

FIGURE 14 
The four sides of this box and the bottom were held together with wood screws so that it could be disassembled for removal of the plastic alveolus after this had been cast and set solid. In order to facilitate the removal of the center core (or stone model of the root) from the hardened plastic, the wooden mold was sectioned longitudinally into two halves. These were pinned together to maintain accurate alignment. This made it possible to cast the alveolus in two halves which could be approximated to form the whole mode1 alveolus.

The center "core" used in the construction of the alveolus was made as follows: (1) the root of the model tooth was covered with a uniform (2 mm.) thickness of red utility wax. The thickness of the wax was checked for uniformity by means of a straight pin inserted into the wax at different places, and a vernier calipers to measure the depth to which the pin had penetrated. The wax did not cover the root all the way up to the cemento-enamel junction of the model tooth; instead it was trimmed to follow the contour of the cemento-enamel junction at a level that was $15 \mathrm{~mm}$. below this anatomical landmark. This was done to simulate the actual condition that exists in the mouth whereby the average distance between the alveolar crest and the cemento-enamel junction is $1.5 \mathrm{~mm}$. 1 (2) impression plaster was used to make an impression of the root of the model tooth with its covering of a uniform thickness of utility wax.

1. Kronfe1d, R.: Denta1 Histology and Comparative Dental Anatomy. Lea and Febiger, Philadelphia, 1937. 
This impression was poured in dental stone and the resulting stone model (Figure 15) was used to form the inside of the alveolus.

Castolite Liquid Plastic ${ }^{1}$ was used for the casting of the model alveolus. This plastic is supplied with a liquid hardener which is mixed into the liquid plastic to initiate the cold-curing (exothermic) reaction. In the solid state it is a water-clear plastic.

As already mentioned above, the wooden mold was in two parts. This allowed the alveolus to be poured in layers. The mold was laid flat on its side and the stone model was supported in the center by means of a dowel wire projecting from its apex and a handle projecting out from the top as illustrated in figure 16A. The Castolite was poured into the mold and allowed to set. When the lower half of the mold was completely cast (and hardened) aluminum foil was adapted to that half of the alveolus to act as a separating medium in the pouring of the second half. The top half of the wooden mold was approximated to the lower half and fixed in position by its pins. The Castolite was mixed and poured in layers into this part of the mold as seen in figure 16B. After this half was completely cast and set, the wooden mold was disassembled by removing the screws that held the sides and bottom together. The two halves of the plastic alveolus were then separated and the "center core" (or stone model) was removed. The model that resulted was in two pieces that fit together against each other; it had the outside shape of a square column

1. The Castolite Company, Woodstock, I11inois. 


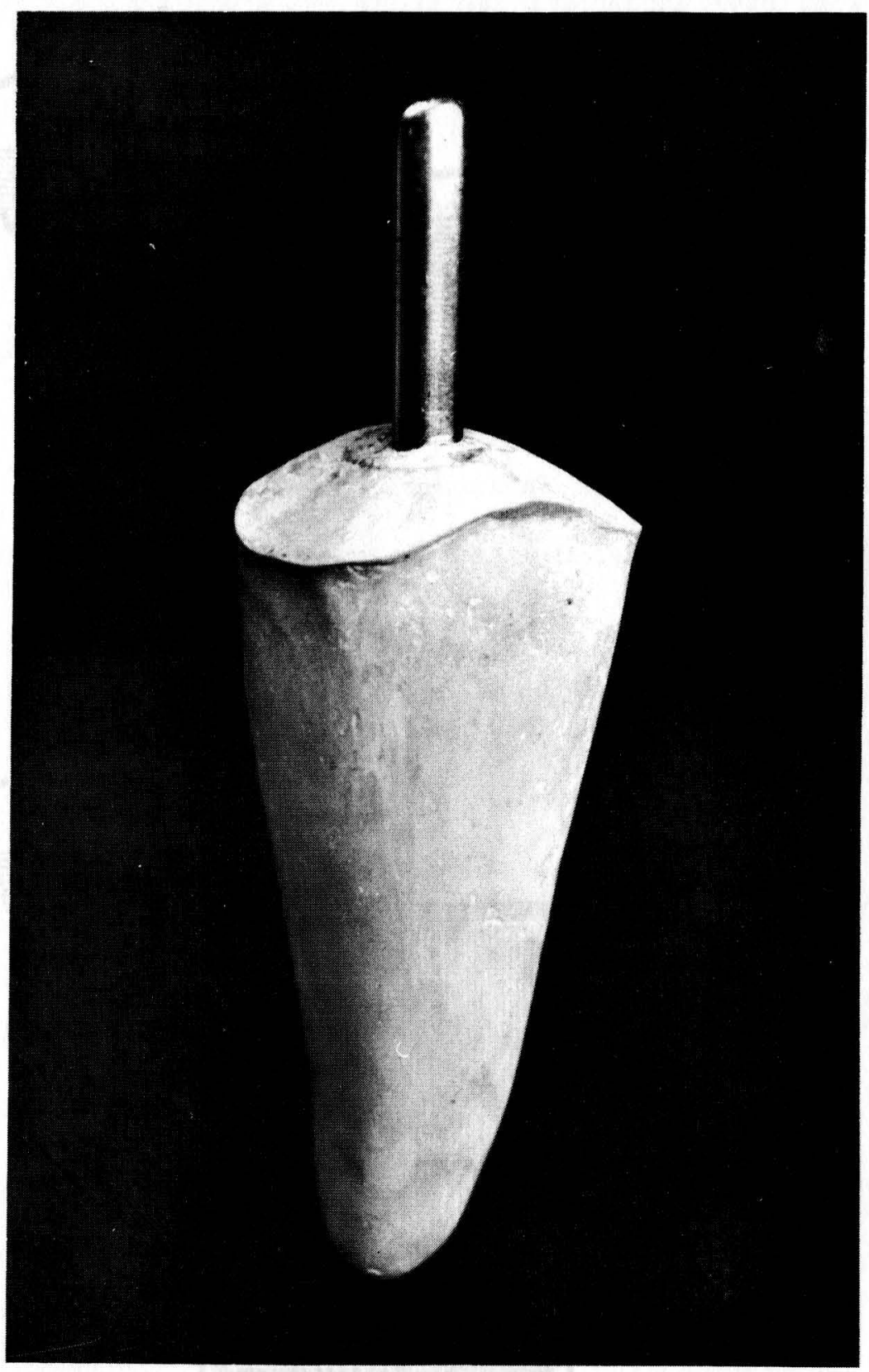

FIGURE 15

MODEL OF THE ROOT USED TO FORM

THE INSIDE SHAPE OF THE ALVEOLUS 

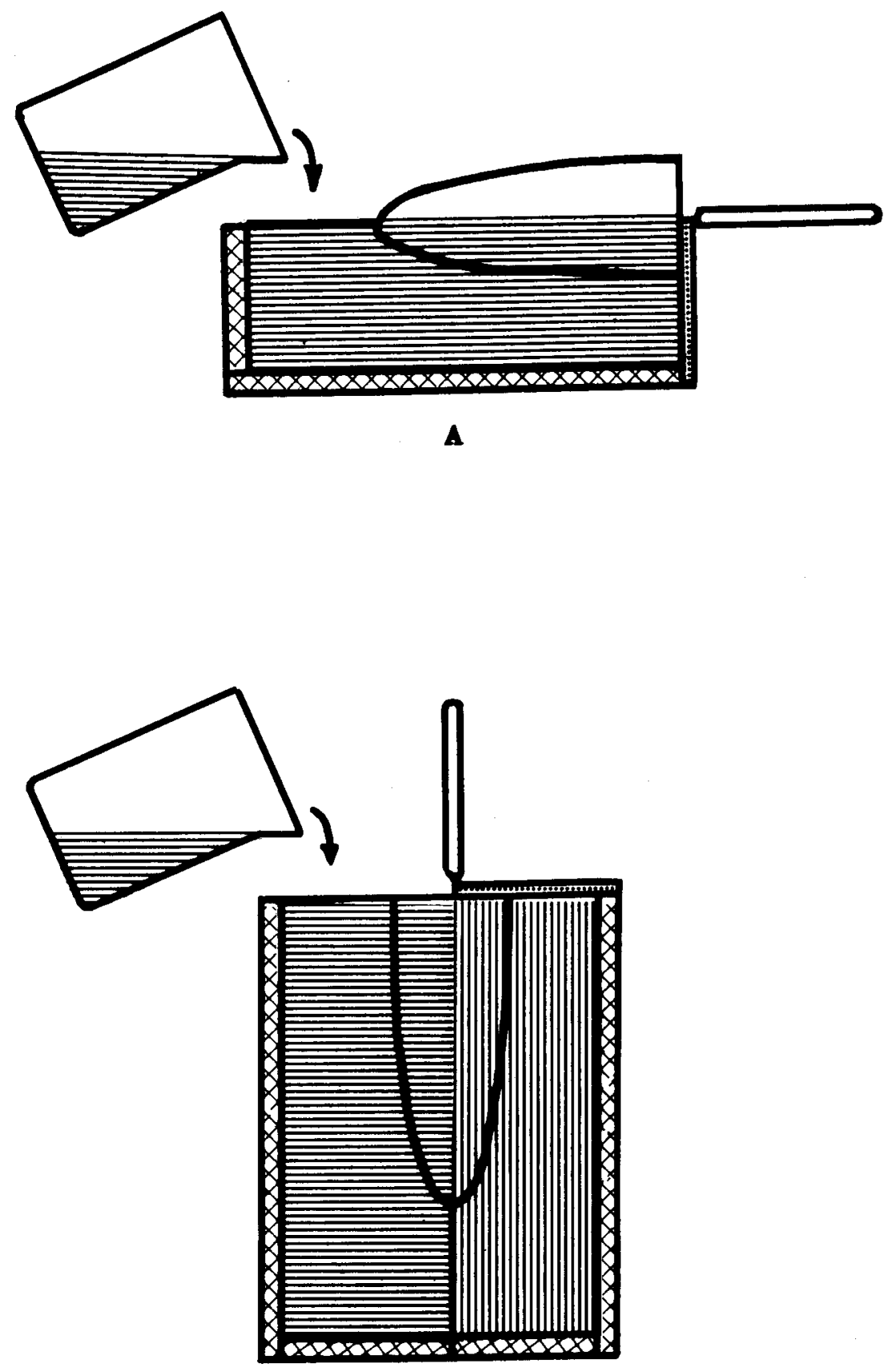

B

FIGURE 16

METHOD OF CASTING THE ALVEOLUS IN TWO STAGES 
$3.5^{\prime \prime} \times 3.5^{\prime \prime} \times 8.0^{\prime \prime}$. The inside shape and dimensions were those of the stone model of the root. The outside surfaces of the alveolus were squared off and trimmed with a power saw, sanded, and polished. The inside surfaces were contoured where necessary and flaws in the castings were repaired as needed.

\section{Construction of the Periodonta1 Ligament Analog}

A Perma-Flex Cold Molding Compound ${ }^{1}$ was used to make a rubber-like webbing that would represent the periodontal ligament of the model tooth. This compound is a black, viscous, polysulfide base, liquid synthetic rubber that is supplied with a curative compound or catalyst. It sets at room temperature and forms a rubbery, flexible, extremely elastic substance.

The model "periodontal ligament" was made in two halves - one half for the mesial surface of the root and the other half for the distal surface. The two pieces of rubber webbing (Figure 17A) consisted of:

(1) strands running longitudinally from the alveolar crest to the apex that were not parallel but converged toward the apex and (2) circumferential strands arranged in such manner as to represent the angulation that the different groups of periodontal fibers make with the root surfaces of the teeth. This is illustrated by the diagram in figure 17B. For examp1e, the "horizontal fibers" of the periodontal ligament around the

1. Trade name; "BLAK-STRECHY". The Perma-Flex Mold Company, Columbus, Ohio. 

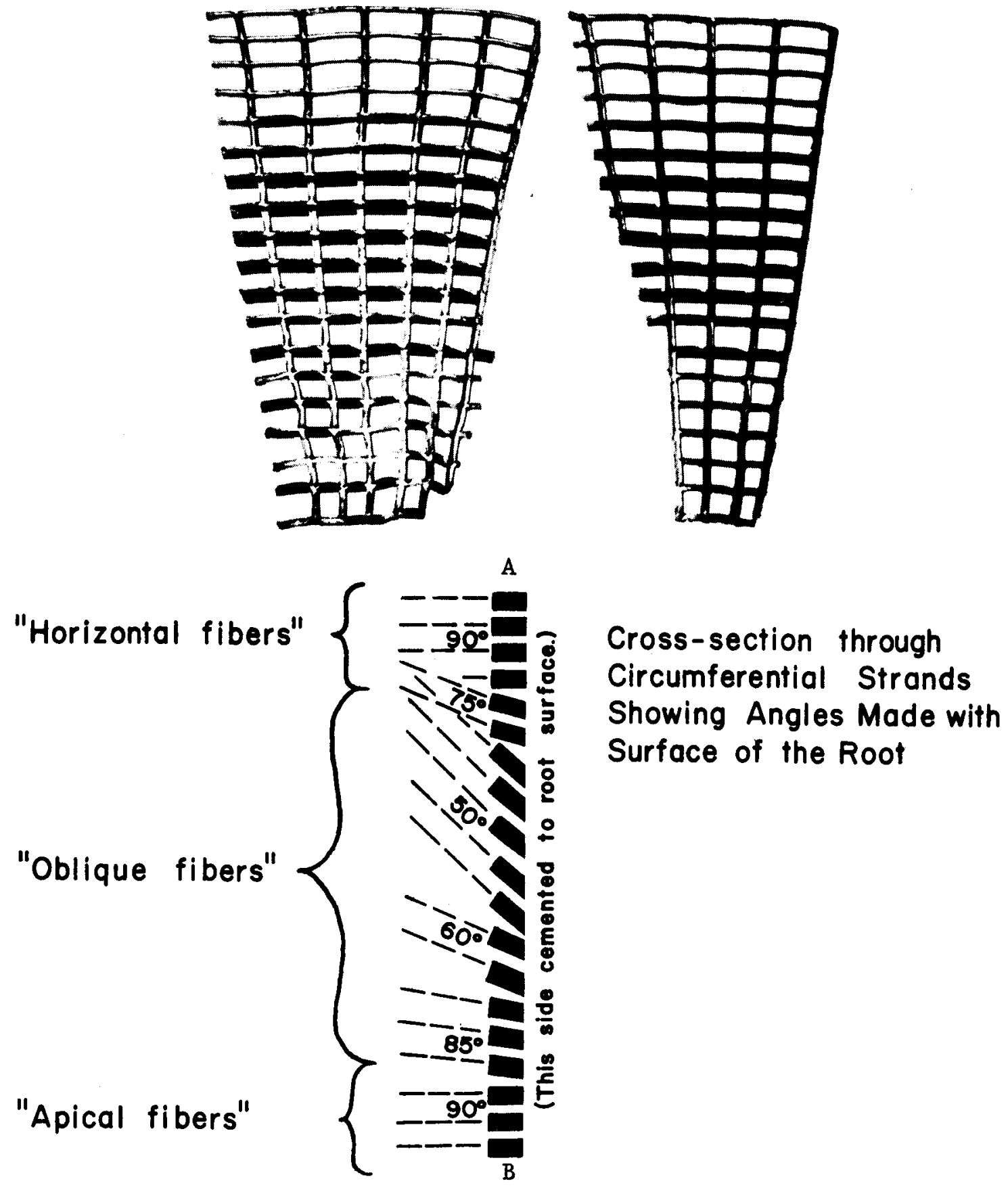

FIGURE 17

RUBBER ANALOG OF THE PERIODONTAL LIGAMENT 
coronal third of the root which are approximately perpendicular to both the root surface and the alveolar crest, are represented on the analog by rubber strands that have the long axis of their cross-section ${ }^{1}$ perpendicular to the root surface. The fibers around the central portion of the roots of teeth form acute angles with the root surfaces and they are called "oblique fibers". These are represented on the model by having the rubber strands (around the center of the root) form acute angles ${ }^{2}$ with the surface of the root. The angles vary from $75^{\circ}$ to $50^{\circ}$ to $85^{\circ}$ at the different levels of the root (Figure 17B).

The two webs or nets of rubber were made in molds that were constructed out of two pieces of sheet Plexiglas. In preparing to make the molds, two paper templates were cut and fitted to the mesial and distal faces of the root. Upon these templates the network of desired rubber strands was drawn. The templates were then transferred to the sheets of 3/8" (thick) Plexiglas and the required grooves were milled into the Plexiglas with a saw blade designed for cutting plastic. The resulting molds are shown in figure 18.

After the molds were cleaned they were coated with a release agent to prevent undue adhesion; then they were set adjacent. The freshly

1. All these strands - both longitudinal and circumferentia1 - were rectangular in cross-section.

2. These angles are averages of measurements that were made on photographs of actual histologic sections of the periodontal ligament. Sicher, H.: Orban's Oral Histology and Embryology. The C.V. Mosby Company, Saint Louis, 1962. 


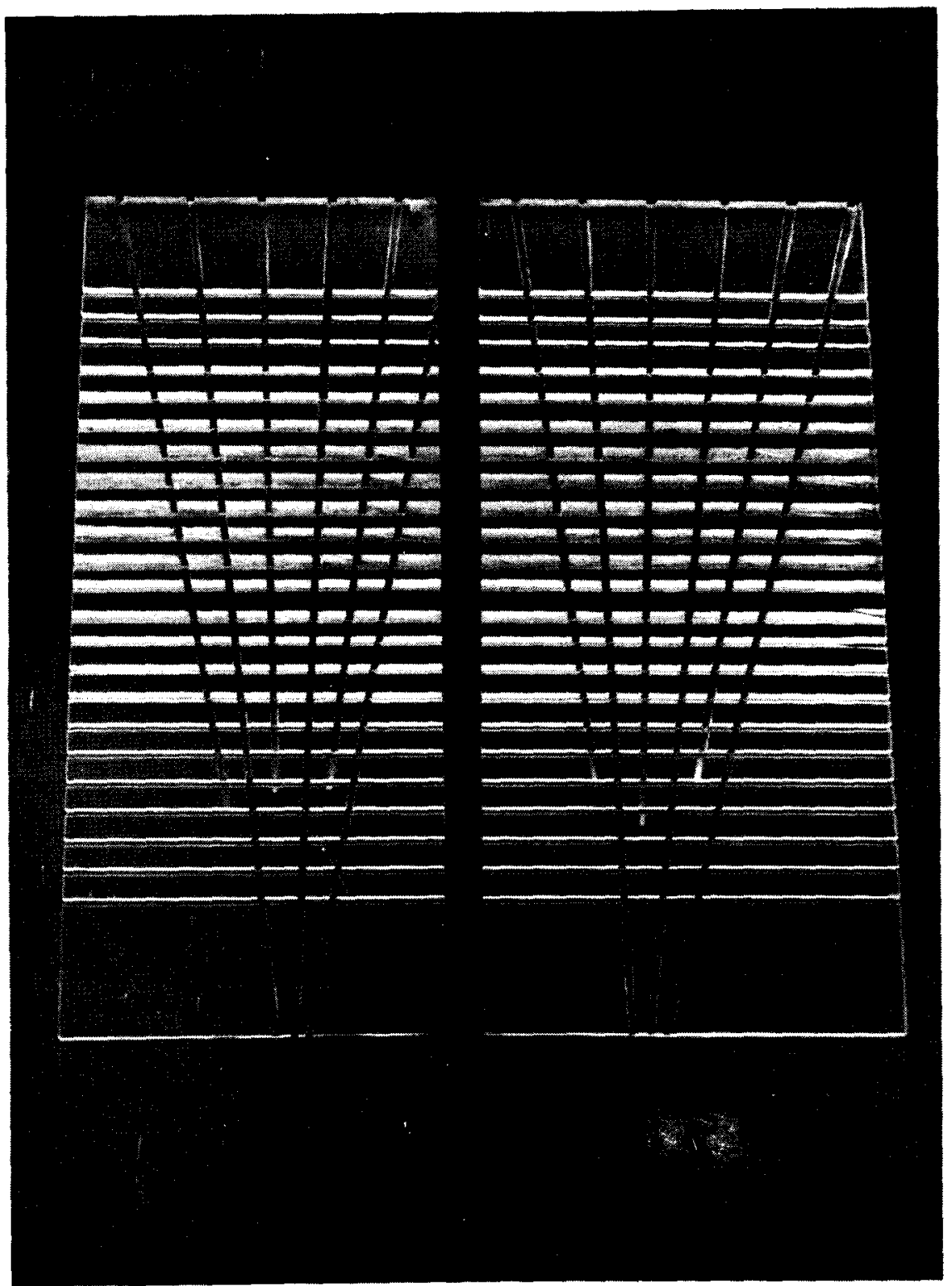

FIGURE 18

PLEXIGLAS MOLDS USED TO FORM

THE ANALOG PERIODONTAL LIGAMENT 
mixed rubber compound was poured onto the molds, spatulated into the grooves, and smoothed so that a very thin layer remained on the flat surface of the mold. After 48 hours, the networks were removed and all of the interstices were cleared. The two networks were attached with Ducco cement to the mesial and distal surfaces of the root of the mode1 tooth (Figure 19).

\section{Methods of Study}

The completed analog of tooth, periodontal ligament and alveolus, was mounted rigidly with bolts onto a plywood assembly having two platforms -- one at the base and one at the level of the crest of the plastic alveolus as seen in figure 20. For maximum stability and rigidity of the entire system, these platforms were fixed to the back of the plywood assembly with glue and long wood screws, and the whole assembly was reinforced with appropriately placed wooden and steel braces. A pulley on an adjustable bracket was placed on each side of the upper platform to allow for the suspending of weights over the sides.

Two dial indicators were used to measure the movements of the tooth in a mesiodistal plane. One was affixed to the lower platform and it contacted the mesial surface of the root through a hole drilled in the plastic alveolus at the level .390 inches up from the apex. The other dial indicator was mounted on the upper platform and it contacted the distal surface of the crown of the tooth at a level 7.379 inches from the apex (or 6.989 inches above the lower dial indicator). The deflections of the crown and those of the root were measured to thousandths of 


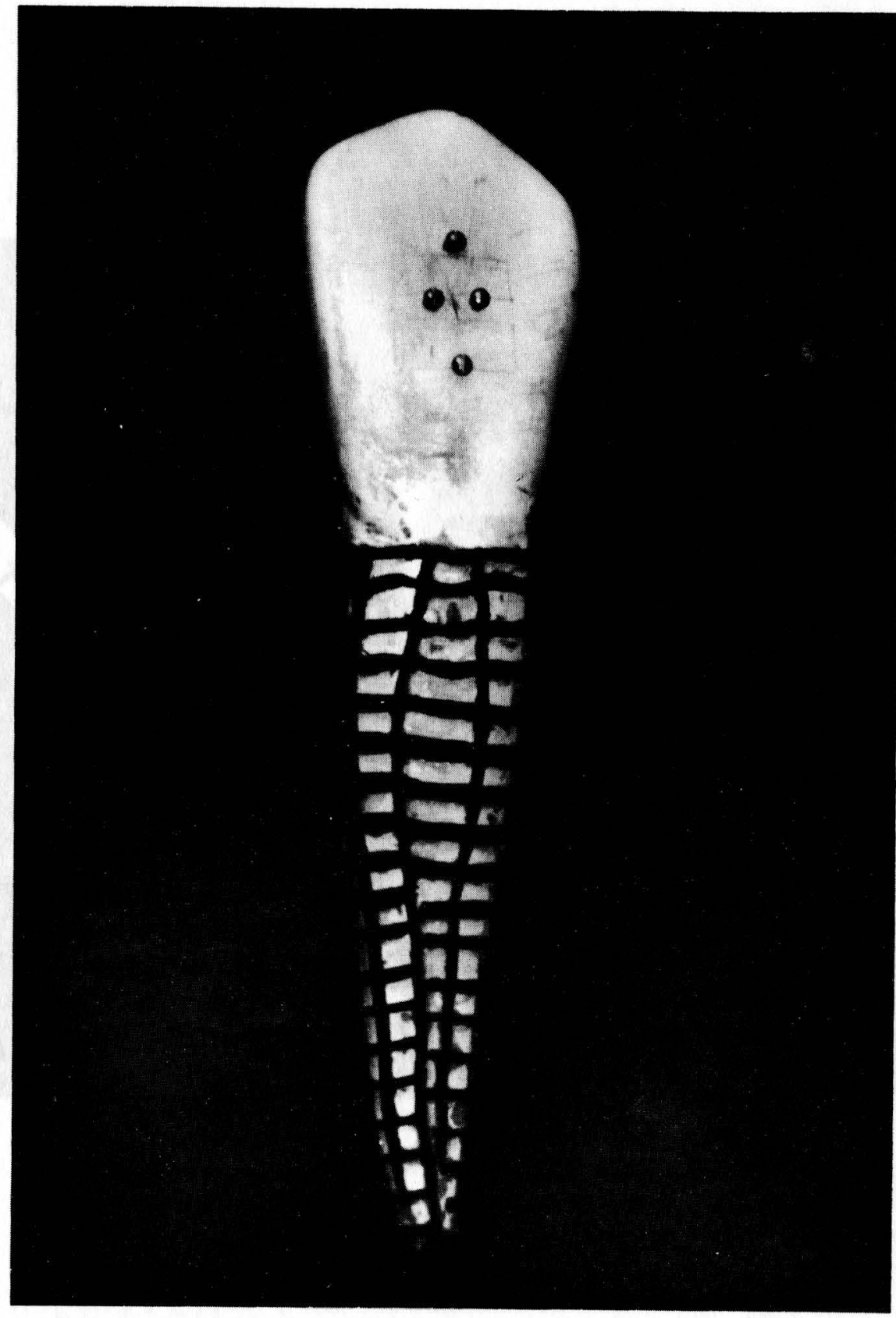

FIGURE 19

MODEL TOOTH WITH ANALOG PERIODONTAL

LIGAMENT CEMENTED TO THE ROOT SURFACE 


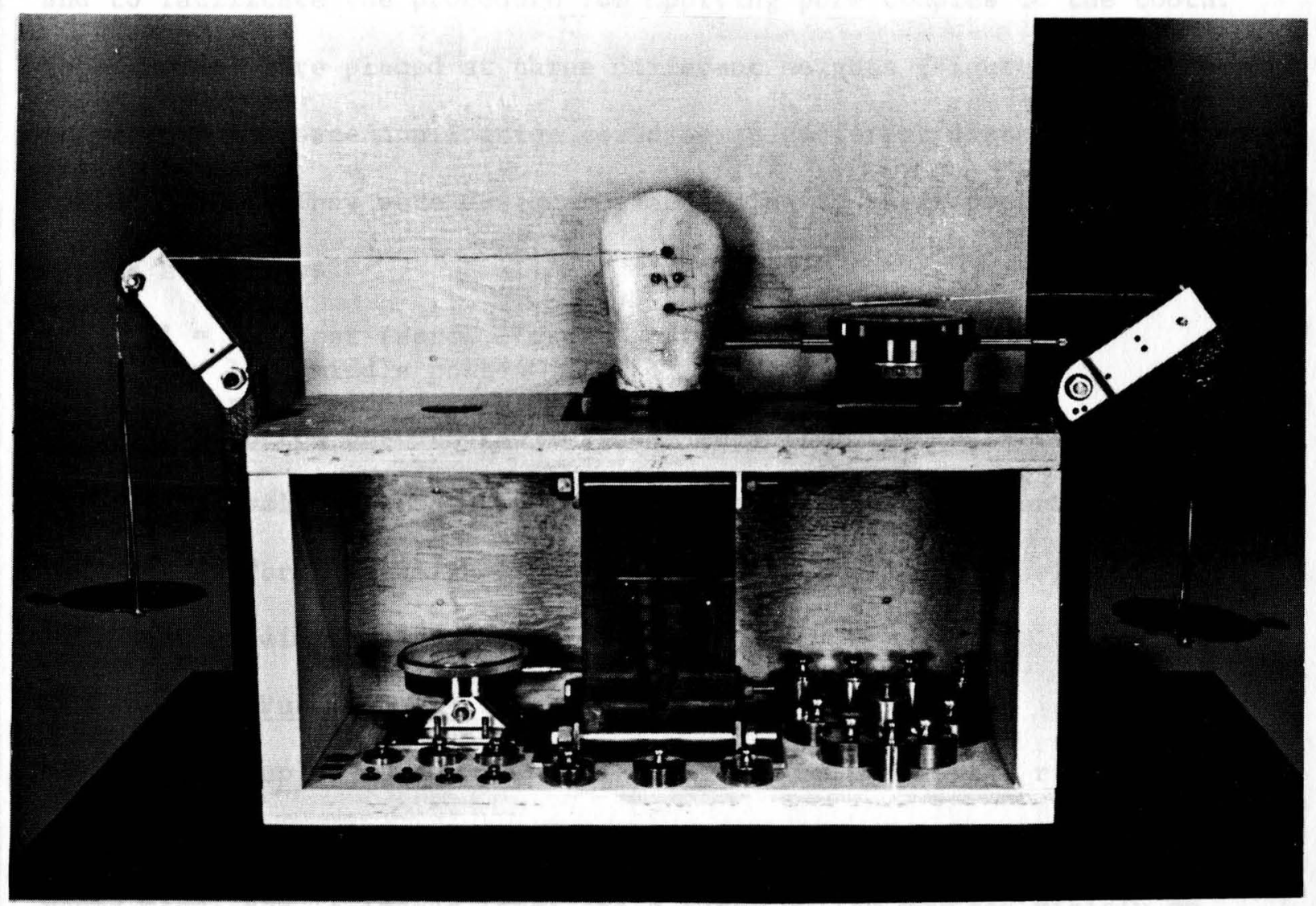

FIGURE 20

COMPLETED ANALOG MOUNTED ON PLYWOOD

ASSEMBLY WITH CROWN AND ROOT DIAL INDICATOR 
a millimeter by the upper and lower dial indicators respectively.

Four screws were inserted into the labial surface of the model tooth in order to serve as posts for the attachment of the weights to the tooth and to facilitate the procedure for applying pure couples to the tooth. These screws were placed at three different heights (Figure 21) so that the points of force application could be at different distances from the incisal edge. They were designated according to their positions on the crowns as follows:

非 = top post (screw closest to the incisal edge)

$\# 2$ = left middle post

非 3 = right middle post

非 4 = bottom post (screw furthest away from the incisal edge)

The three levels selected for the screws corresponded proportionately to the various "bracket heights" that are possible clinically with the different banding techniques.

E. Applying Pure Couples to the Analog

Pure couples were applied to the tooth by means of a rig that consisted of a flexible steel blade -- 3 inches wide and 31 inches 1ong; a round steel rod 15 inches long; and a large pulley mounted rigidly on the steel rod. Figure 22 illustrates the manner in which a moment of force was applied to the steel rod by suspending weights from the large pul1ey. It a1so demonstrates how this moment of force was transmitted through the flexible steel blade to the tooth where it was expressed as a couple because of the two-point contact that the blade made with two of the screws (非1 and 非) on the labial surface of the tooth. The 


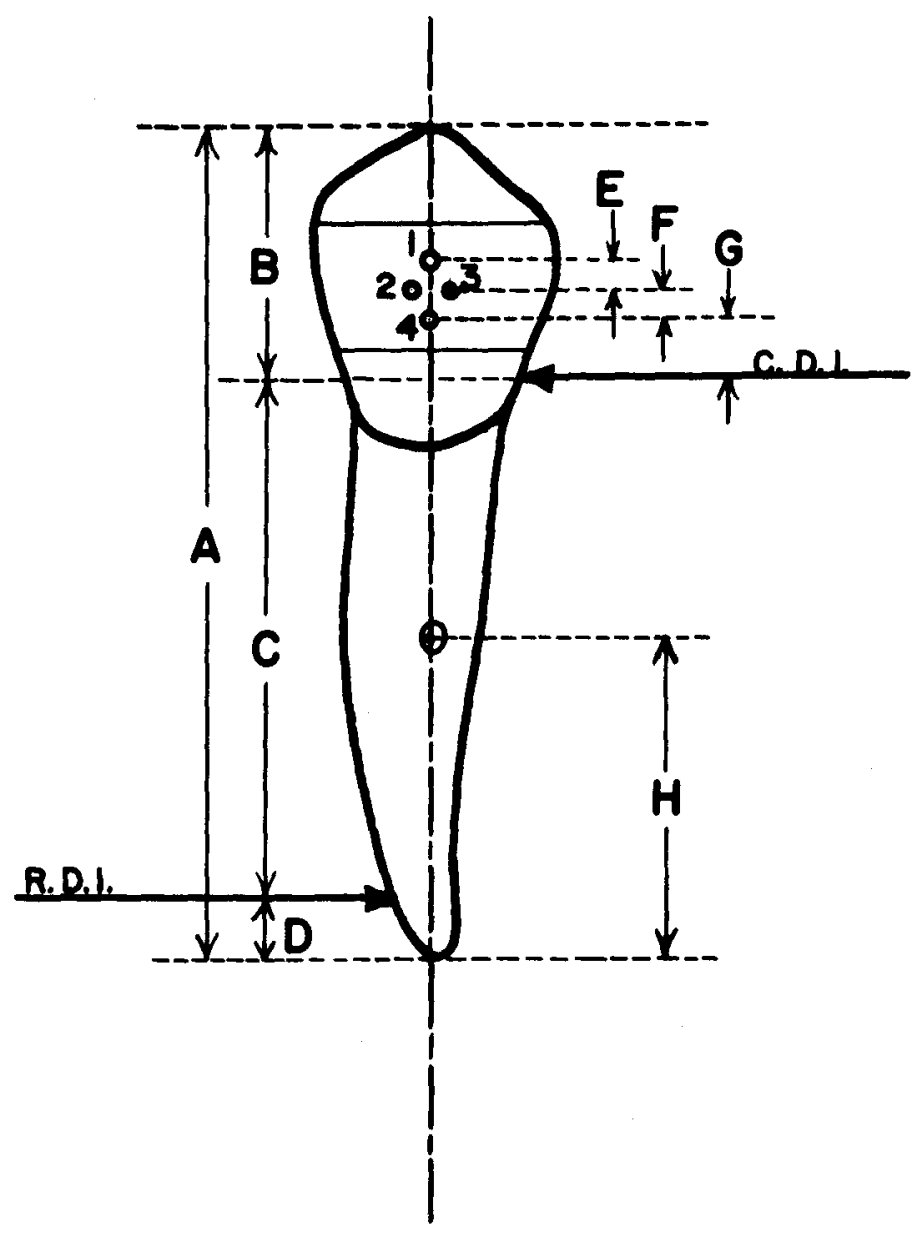

$A=$ total height of tooth

$B=$ incisal edge to Crown Dial Indicator

$C=$ Crown Dial Indicator to Root Dial Indicator

$D=$ Root Dial Indicator to root apex

$E=$ Post No.l to Post No.2

$F=$ Post No. 2 to Post No. 4

$G=$ Post No.4 to Crown Dial Indicator

$H=$ root apex to Center of Tipping (O) 10.594in.

3.215 in.

$6.989 \mathrm{in}$.

$.390 \mathrm{in}$.

.576 in.

$.608 \mathrm{in}$

830 in

(variable)

FIGURE 21

DIAGRAM OF HEIGHTS OF IMPORTANT

POINTS ON THE MODEL, TOOTH 
<smiles>[Te][Te]</smiles> 
magnitude of the couple being applied to the tooth at any particular time was exactly the same as the magnitude of the moment being generated by the weights on the pulley. The length of the moment arm in this case was 4.078 inches, which was the radius of the pulley. This was determined by measuring the outside diameter of the pulley with calipers and dividing this diameter by two.

Seven different weights were suspended from the pulley at separate intervals and readings of crown deflection and root deflection were taken each time after $21 / 2$ minutes had been allowed to elapse. The magnitude of the moment producing the deflections was calculated by multiplying the weight used by the moment arm (4.078 inches) and this was recorded next to the corresponding deflections as seen in the Data Sheet in Appendix I. The weights used ranged from one-half pound to four pounds (inclusively) with an increment of one-half pound each time. After recording the deflections produced by each particular couple, the weights were removed to allow the rubber to return to its original shape and bring the tooth back to zero readings on the dial indicators. Then the next weights were applied to the pulley, and the same procedure was repeated.

F. Applying Tipping Forces to the Analog

In order to produce tipping of the model tooth within the limits of its alveolus, single forces of known magnitude and direction were applied to the crown. The point of force application was also known in every instance since the screws in the labial surface of the tooth were 
used as the posts for the application of the forces and the positions of these screws (relative to the total height of the tooth) had already been predetermined.

The first set of data was taken with the weights attached to the top post (非). Orthodontic steel ligature wire (.012 in.) was used to attach a tray to the top post on the tooth. A suitable length of wire was used so that the tray could extend beyond the pulley at the right end of the platform and hang suspended over the side (Figure 20). Since the height of the pulley was adjustable, the direction of pull of the wire could be controlled. A level was used to set the wire horizontal to the floor. This made the direction of pull approximately perpendicular to the long axis of the tooth since the tooth and its alveolus were set upright in the platform assembly.

The weights used in this part of the experiment ranged from onehalf pound to five pounds (inclusively) with an increment of one-half pound each time. Thus, a total of ten single forces were applied in a distal direction to the top post on the crown of the tooth. The deflections of the crown and those of the root were measured by the upper and lower dial indicators respectively and recorded on the data sheet next to the weight that produced them. Readings of the deflections were taken after each of the particular weights had been allowed to act on the tooth for a period of $21 / 2$ minutes. To test the accuracy of the data, the entire procedure was repeated three times and the corresponding data were designated: Trial A, Trial B, and Trial C. 
When all the data had been obtained for forces applied to the top post, the wire was changed to one of the middle posts (非) and it was made parallel to the floor by adjusting the height of the pulley. The exact same procedure as already described for the top post was followed in obtaining the data for the middle post, and the experiment was repeated three times (Trials $A, B, C$ ) using the same weights and the same time interval as before.

After al1 the data had been recorded for post 非, the wire was then attached to the bottom post (非) and the experiment was repeated another three times as described above. A11 of the data obtained were analyzed according to Fisher's Analysis of Variance.

G. Producing Bodily Movement of the Model Tooth

In order to produce bodily movement of the model tooth distally (within the limits of its alveolus) a force and a couple had to be applied to the crown of the tooth. This was achieved by utilizing the pulleys at both ends of the upper platform and by attaching appropriate weights to the top and bottom posts (非 and $\# 4$ ) on the crown. The tooth was considered to have translated dista11y (or moved bodily) when the upper and lower dial indicators measured precisely the same deflection in the same direction for both the crown and root.

The particular combination of force and couple that would be required to produce bodily movement of the tooth against the resistance of the rubber "periodontal ligament" could have been determined experimentally by the process of trial and error, or calculated mathematically if 
the location of centroid of effective root area were known. The latter method was selected because it was more practical and convenient.

The mathematical calculations that were used in the location of the centroid of area and in the determination of the force system needed to produce pure translation will be described in Chapter $V$ and discussed in Chapter VI. From these equations, it was possible to predict that a force of 6.6 pounds acting on the top post (非) in a mesial direction (toward the left) in conjunction with a force of 8.5 pounds acting on the bottom post (\#4) in a distal direction (toward the right) would produce bodily movement distally if both forces were parallel to each other and perpendicular to the long axis of the tooth.

To test the accuracy of these calculations and the validity of the prediction that was made from them, the experimental procedure was conducted as follows: (1) a weight of 6.6 pounds was attached to the top post and suspended over the left side of the platform. Its action line was made parallel to the floor with the aid of a level; (2) the weight of 8.5 pounds was bracketed in include plus and minus one pound, i.e., a range of weights that included $7.5,8.0,8.5,9.0$ and 9.5 pounds was attached to the bottom post and suspended over the right side of the platform. The action line of these forces was also made parallel to the floor; (3) readings of the crown and root deflections were taken after each of the weights applied to the bottom post had been suspended for $21 / 2$ minutes. 


\section{CHAPTER V}

\section{EXPERIMENTAL RESULTS}

\section{A. Appraisal of Tipping Produced by Pure Couples}

The first data that were gathered in this experiment related to the behavior of the tooth under the influence of pure couples acting on the crown. As noted in Chapter IV under the description of methods of applying pure couples to the analog, the rig was so designed that only pure couples would act on the tooth. This consideration was important since it was known that a pure couple acting anywhere on the tooth would cause the center of rotation (or tipping) to be at the centroid of the tooth.

A11 the couples were in a clockwise direction in relation to the labial surface of the tooth. Invariably, they produced a distal tipping of the model tooth, that is, the crown moved distally in every instance while the apical portion of the root moved in the opposite direction (mesially). As the magnitude of the couple was increased in successive trials from 2.039 to 16.312 pound-inches, the deflections of both the crown and the root increased proportinnately. In other words, the greater the magnitude of the applied couple, the greater was the degree of distal tipping. These same characteristics and results were obtained when the entire experiment was repeated a second time.

The amounts of crown and root deflections produced by each couple 
in both Trials A and B, are seen in Appendix I. The apparent center of tipping in each instance was determined mathematically according to the calculations shown in Appendix II. The graph in figure 23 was prepared to illustrate the changes that occurred in the location of the apparent center of tipping as the magnitude of the tipping couple increased.

\section{B. Appraisal of Tipping Produced by Single Forces}

The application of single forces (of different magnitude but constant direction) to the crown of the model tooth produced crown and root deflections that were characteristic of tipping, that is, the crown moved in the direction of the force (distally) while the root moved in the opposite direction. Changing the magnitude of the applied force had definite effects on the degree of the crown and root deflections as well as on the position of the center of tipping. When these same forces were again applied to the tooth, but at a different level of the crown, the deflections of the crown and root and the location of the center of tipping changed. The data collected in this part of the experiment are shown in Appendices III, IV, and V.

The direction of the single forces was kept constant throughout all the trials, that is, all the forces acted parallel to the floor in a distal direction regardless of the magnitude or point of application. Thus, there were two variables in this part of the experiment: force magnitude and point of application.

\section{The Effects of Changing Force Magnitude}

The graph in Figure 24 shows that as the applied force increased in 


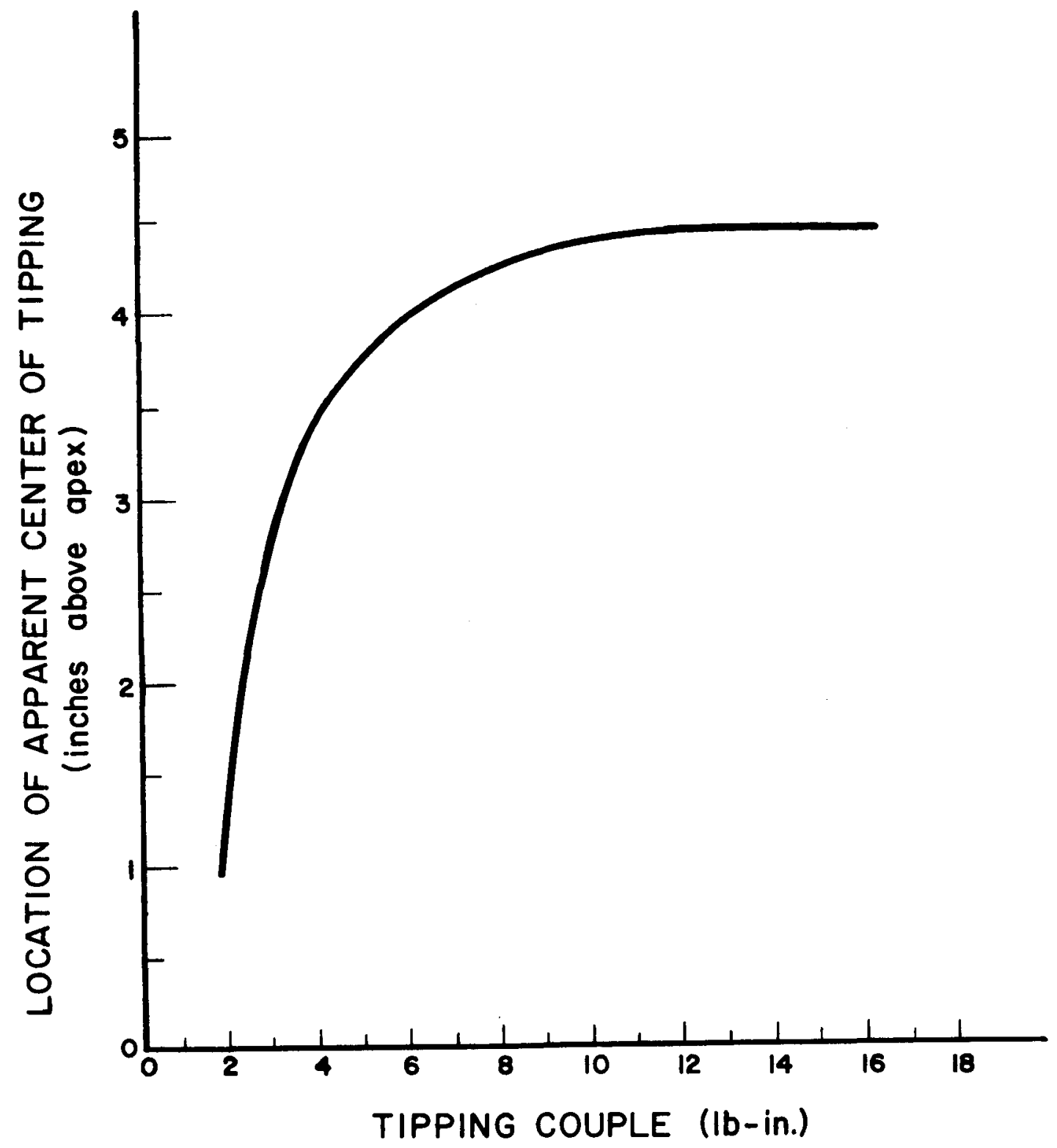

FIGURE 23

GRAPH OF APPARENT CENTER OF TIPPING

VS. TIPPING COUPLE 


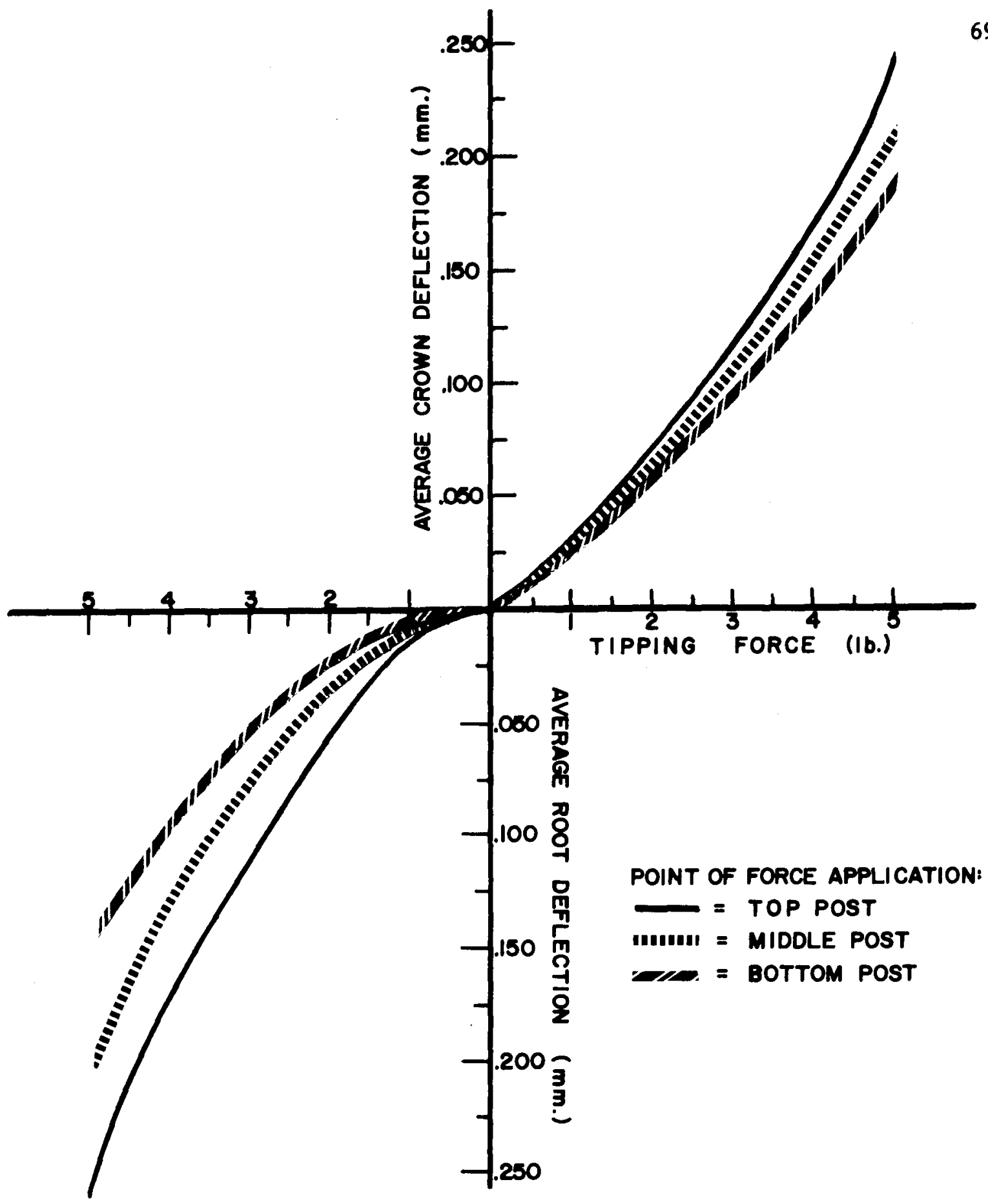

FIGURE 24

GRAPH OF CROWN AND ROOT DEFLECTIONS

VS. TIPPING FORCE 
magnitude from one-half pound to five pounds the deflections of both the crown and the root also increased uniformly. The range of deflections was different for the different points of application but, nonetheless, the increase in the deflections was similar in all three cases. The curve in black demonstrates the increase in deflections when the forces were applied at the top post (非) on the crown of the tooth. The blue and the red curves show the relationship of force vs. deflection when the forces were applied at the middle (非) and bottom (非) posts respectively.

The distal tipping produced by each single force was evaluated individually. The apparent center of tipping in each instance was determined mathematically according to the calculations shown in Appendix II. The distance up from the apex of each apparent center of tipping was plotted against the particular force magnitude that produced the tipping. The changes that occurred in the position of this center of tipping are shown in the graph in figure 25.

\section{The Effects of Changing Point of Application}

As already noted above, the graph in figure 24 shows that deflections increased uniformly as force magnitudes increased. For a particular force magnitude, however, the deflections were different for each of the three points of force application. The deflections were greatest when the single forces were applied to the top post (非) and least when the forces were applied to the bottom post (非). This is also represented on the composite graph of all data seen in figure 24 .

The location of the apparent center of tipping was calculated for 


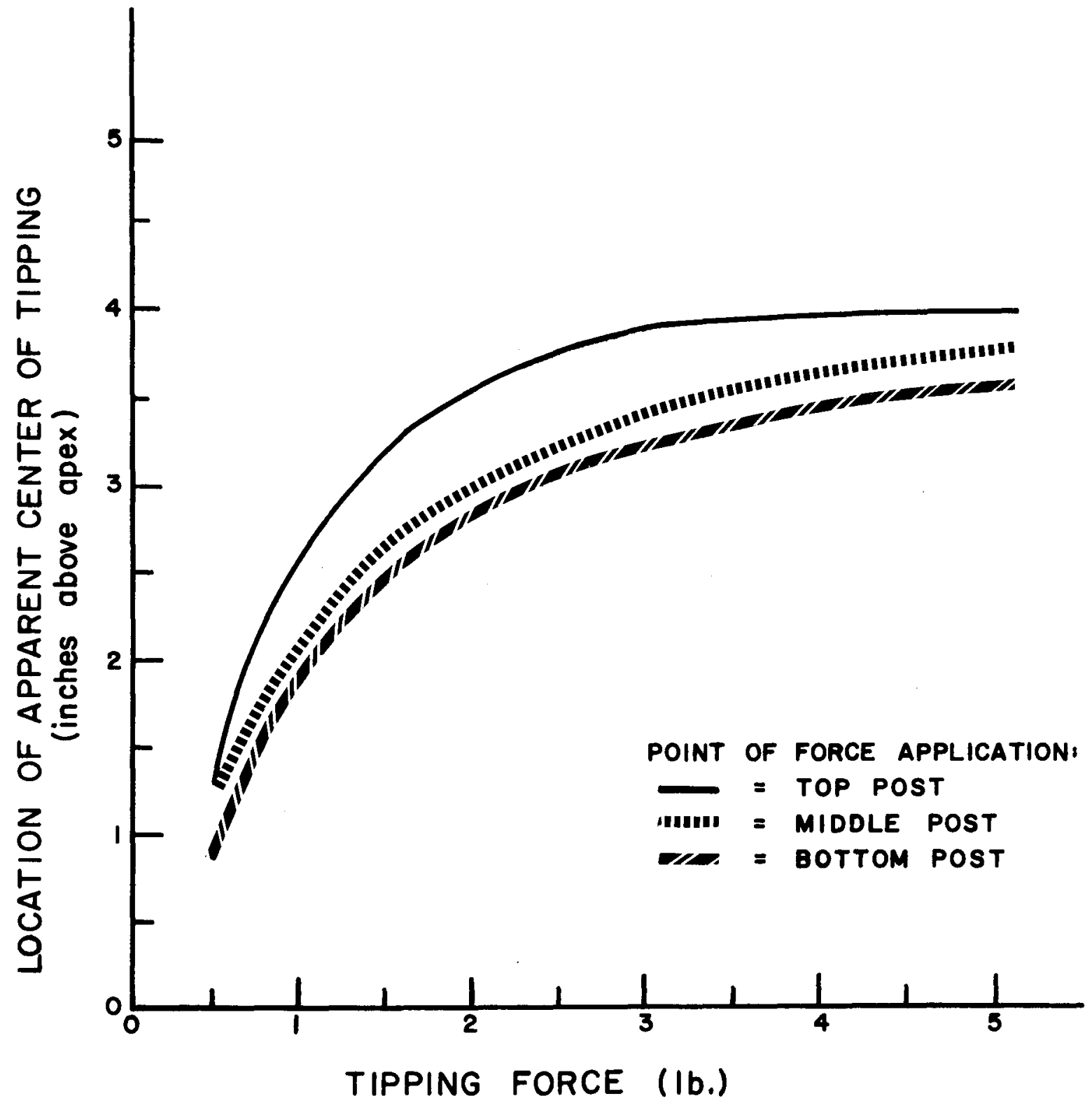

FIGURE 25

GRAPH OF APPARENT CENTER OF TIPPING

VS. TIPPING FORCE 
each individual tipping situation by following the same mathematical procedures described in Appexdix II. The effect that the point of application had on the center of tipping is shown graphically in figure 25 by the different levels of the curves. The apparent centers of tipping were always more cervical when the forces were applied to the top post (非) and closest to the apex when they were applied to the bottom post (非).

\section{Statistica1 Analysis of the Data}

The measurements of deflection of the crown of the model tooth were analyzed by Fisher's Analysis of Variance with the results shown in table I. As mentioned previously, the sources of variation were force magnitude (weights), and points of application (positions). Another source of variation (trials) was introduced by the repetition of the experiment on three separate occasions. The usua1 2-factor and 3-factor interactions were calculated and their sums of squares are also 1 isted in table $I$. The mean square for the $\mathrm{T} \times \mathrm{W} \times \mathrm{P}$ interaction, when combined with the mean square for the $\mathrm{T} \times \mathrm{W}$ interaction yields a new mean square of .00002066 which will serve as an estimate of experimental error. Both of the other interactions were found to be significant when tested against this estimate of experimental error. The mean squares due to the different weights and due to the different points of attachment of the weights were significantly large. There was, however, no significance attached to the mean square due to the different trials.

The measurements of deflection of the root were also analyzed according to Fisher's Analysis of Variance and the results obtained are 
TABLE I

ANALYSIS OF VARIANCE

(CROWN DEFLECTIONS)

\begin{tabular}{|c|c|c|c|c|c|}
\hline SOURCES & D.F. & S.S. & M.S. & F. & SIGNIFICANCE \\
\hline \hline TRIALS & 2 & .000071 & .0000355 & 1.72 & $3.17(5 \%)$ N.S. \\
\hline WEIGHTS & 9 & .353870 & .0393189 & 1902.50 & $2.75(1 \%) * * *$ \\
\hline POSITIONS & 2 & .008631 & .0043155 & 26.23 & $18.00(1 \%) * *$ \\
\hline T X W & 18 & .000417 & .0000232 & & $2.68(1 \%) * *$ \\
\hline T X P & 4 & .000658 & .0001645 & 7.96 & $2.29(1 \%) * * *$ \\
\hline W X P & 18 & .004651 & .0002584 & 12.50 & \\
\hline T X W X P & 36 & .000699 & .0000194 & & \\
\hline TOTAL & 89 & .368997 & & &
\end{tabular}

Standard Deviation of Error $=.0045 \mathrm{~mm}$.

$99 \%$ CONFIDENCE LIMITS are $\pm(2.67 \times .0045)= \pm .012 \mathrm{~mm}$.

D. F. = Degrees of Freedom

S.S. = Sums of Squares

M.S. = Means of Squares

*** = Highly Significant Variance Ratio

N.S. = Non-significant Variance Ratio 
shown in table II. The sources of variation were the same as for the crown measurements, viz., trials, weights and positions. The usual 2-factor and 3-factor interactions were calculated and their sums of squares are listed in table II. The mean square for $\mathrm{T} \times \mathrm{W} \times \mathrm{P}$, when combined with the mean square for the $T \times W$ interaction, yields a new mean square of .0000256 which will serve as an estimate of experimental error. Both of the other interactions were found to be significant when tested against this estimate of experimental error. The mean squares due to the different weights and due to the different points of attachment of the weights were significantly large. There was, however, no significance attached to the mean square due to the different trials.

The two estimates of experimental error (error variance) were so nearly alike as to confirm the belief that the distributions of errors shown by the two gauges were alike. From these mean squares it is possib1e to estimate the size of the distribution of experimental errors. The combined mean square is about .000023 and hence the standard deviation is about .0048 millimeters. The $99 \%$ confidence 1 imits of such distribution are plus and minus .012 millimeters (Figure 26). The lower dial indicator was graduated in .001 millimeters per division and the upper dial indicator was read to the nearest thousandth of a millimeter. "In effect the least count of the measuring indicators was about .001 and therefore the $99 \%$ confidence 1 imits were between 12 and 13 times the least count of the measuring instruments. This is not an unacceptable magnitude although it is a rather sma1l value. 
TABLE II

ANALYSIS OF VARIANCE

(ROOT DEFLECTIONS)

\begin{tabular}{|c|c|c|c|c|c|}
\hline SOURCES & D.F. & S.S. & M.S. & F. & SIGNIFICANCE \\
\hline TRIALS & 2 & .000350 & .000175 & 1.72 & $3.17(5 \%)$ N.S. \\
\hline WEIGHTS & 9 & .378364 & .042040 & 1641.41 & $2.75(1 \%) * * *$ \\
\hline POSITIONS & 2 & .038430 & .019215 & 62.38 & $18.00(1 \%) * *$ \\
\hline T X W & 18 & .000483 & .000027 & & N.S. \\
\hline T X P & 4 & .001232 & .000308 & 12.03 & $3.68(1 \%) * *$ \\
\hline T X X X P & 36 & .000898 & .000025 & & \\
\hline
\end{tabular}

Standard Deviation of Error $=.00506 \mathrm{~mm}$.

$99 \%$ CONFIDENCE LIMITS are $\pm(2.67 \times .00506)= \pm .0135 \mathrm{~mm}$.

D.F. = Degrees of Freedom

S.S. = Sums of Squares

M.S. = Means of Squares

*** $=$ Highly Significant Variance Ratio

N.S. = Non-significant Variance Ratio 


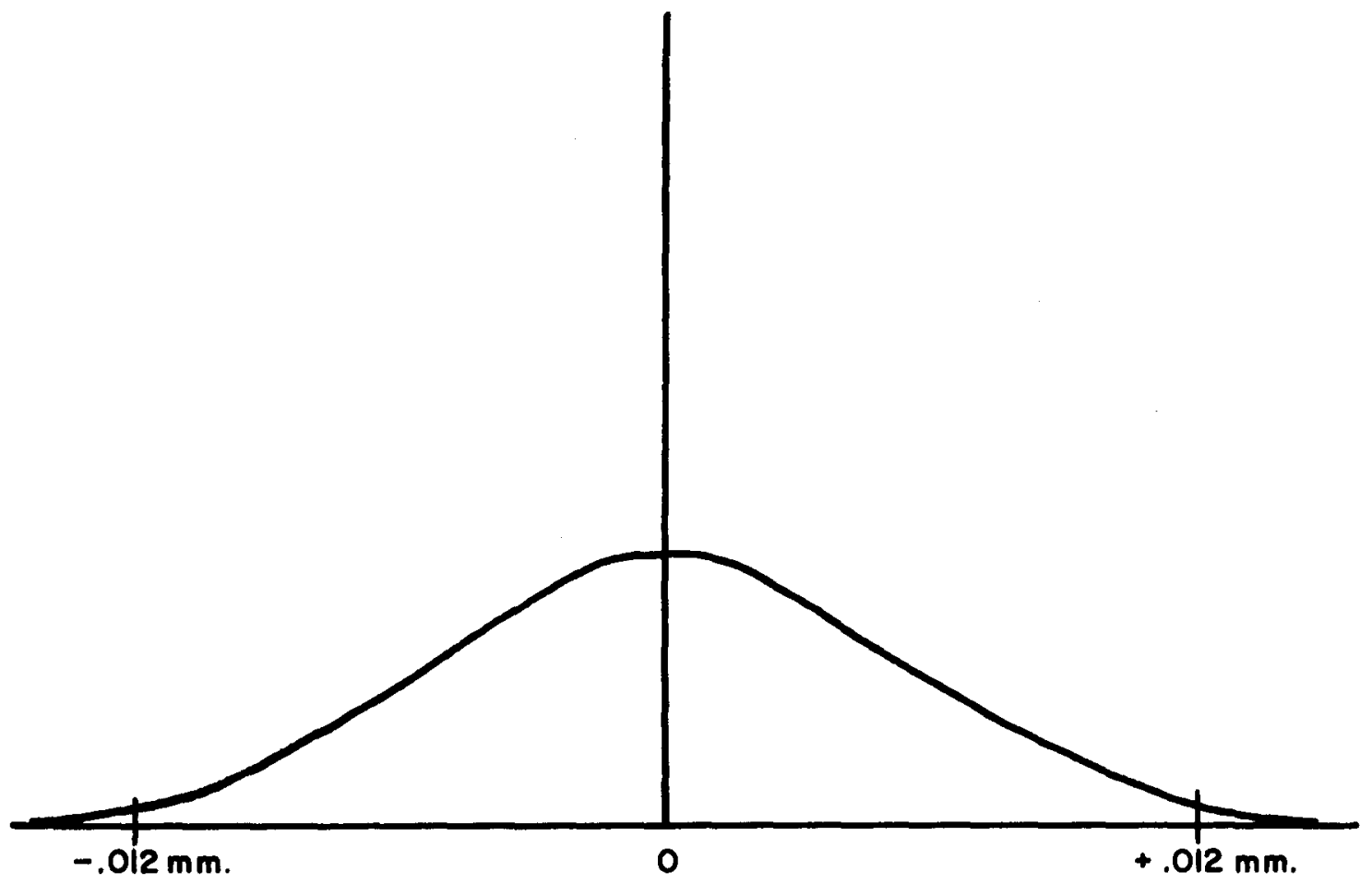

\section{FIGURE 26}

GRAPH OF DISTRIBUTION OF EXPERIMENTAL ERROR 
C. Appraisal of Bodily Movement Produced by a Force and a Couple

Figures 21 and 27 provide the dimensions of the model tooth and the equations of equilibrium for bodily movement. The force $F_{t}$ was arbitrarily set at 6.6 pound for convenience. By solving the equations of equilibrium, $F_{m}$ was found to be 8.577 pounds where the location of centroid was considered to be 4.229 inches above the apex.

The graphs in figure 28 show that crown and root were equally displaced from their rest position when $F_{m}$ was 8.75 pounds. The disagreement from the calculated value was 0.173 pounds. 


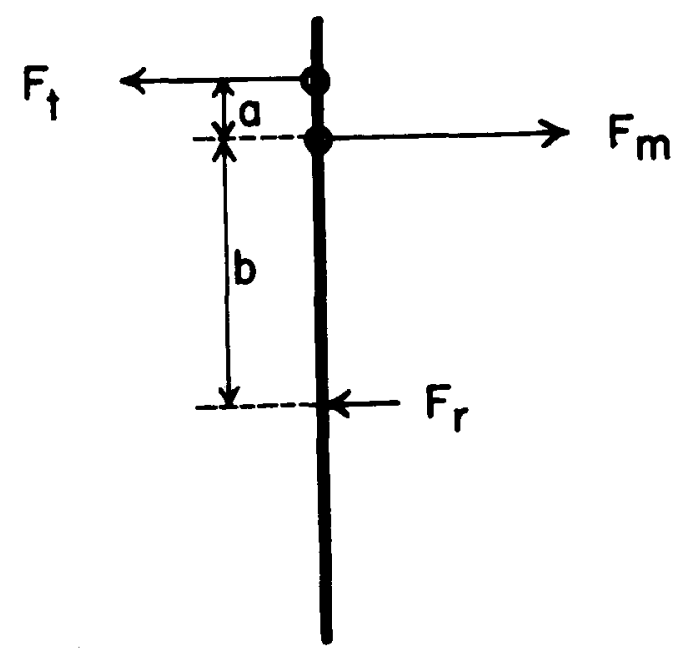

$F_{m}=$ Motivating Force

$F_{m}-F_{f}-F_{r}=0$
$F_{f} \times a-F_{r} \times b=0$

$F_{\uparrow}=$ Counter-acting Force

$F_{r}=\begin{gathered}\text { Resultant of resisting } \\ \text { forces (at Centroid) }\end{gathered}$

$a=1.184$ inches

$F_{m}=F_{f}+F_{r}$

$b=3.980$ inches

$$
\begin{aligned}
& =F_{f}+F_{f}\left(\frac{a}{b}\right) \\
& =F_{f}\left(1+\frac{a}{b}\right) \\
F_{m} & =1.297 F_{f}
\end{aligned}
$$

FIGURE 27

VECTOR DIAGRAM AND EQUATIONS OF EQUILIBRIUM

REPRESENTING THE FORCE SYSTEM NECESSARY

TO PRODUCE BODILY MOVEMENT OF THE MODEL TOOTH 


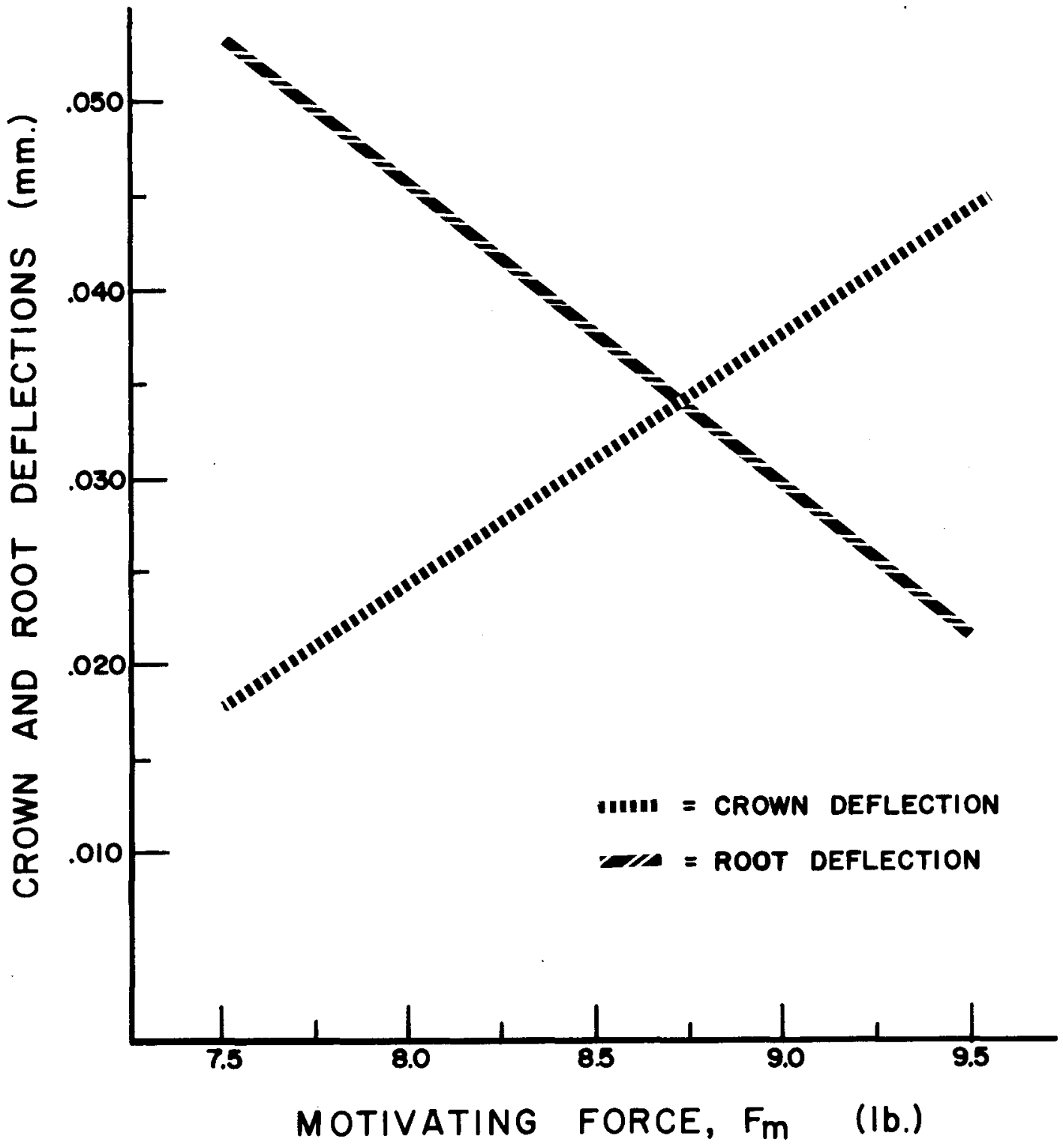

FIGURE 28

GRAPH OF CROWN AND ROOT DEFLECTIONS

VS. MOTIVATING FORCE, $\mathrm{F}_{\mathrm{m}}$ 


\section{DISCUSSION}

Biophysical considerations are rapidly replacing the trial and error method of constructing orthodontic appliances. Physical and mathematical models have been utilized to great advantage to provide needed information regarding the mechanics of orthodontic forces and tooth movements. The newer concepts in the area of biophysics have been advanced in recent years by Graber (1961), Burstone (1962), Haack and Weinstein (1963), and Jarabak and Fizzel1 (1963). Their theories and observations, in particu1ar, were the forerunners of this study.

This research deals with the assessment of the physical aspects of a biophysical process unique to the teeth and the periodontal ligament. The biophysical process consists of the bone resorption and apposition that occurs when a force or force system is applied to a tooth by means of orthodontic appliances. An assessment of the physical aspects of this process includes: (1) an analytical evaluation of the forces, moments and couples, themselves, (2) a study of the tooth movements that result, and (3) an appraisal of the reaction forces and the stresses that develop to resist the tooth movements. In order to study these physical phenomena, it was necessary to design and construct an analog of a tooth and its periodontal environment. This discussion will relate to other models in 
the literature, to this analog and to the findings obtained from the use of this assembled analog.

In order to maintain continuity and orderliness, the experimental results will be discussed in the same order that was established in the preceding chapters: (1) the location of the centroid of effective root area, (2) an assessment of tipping, and (3) the phenomenon and appraisal of bodily movement.

Location of the Centroid of Effective Root Area

In physics, the term "centroid", when used alone, presupposes the center of mass of a three-dimensional object. In the present study, however, this term will always refer to the centroid of effective root area (as described in the Glossary), even when the word "centroid" is used alone for the sake of brevity.

The method employed to locate the centroid of the model tooth involved the application of pure couples to the crown of the tooth. Since a couple consists of two coplanar, non-collinear forces that are equal and opposite, their resultant is always zero. Consequently, a couple has no effect in producing translation of the body on which it acts; it can only produce (or resist) rotation. An important consideration is the fact that a body acted on by a couple can be kept in equilibrium only by another couple of the same moment and in the opposite direction. Thus, if a tooth is acted upon by a single pure couple, its rotation (tipping) is resisted by an equal and opposite couple acting on the root. This counter-couple is the sum of all the stresses, developed within the periociontal 
environment, which act against the root. In the case of the mode1 tooth with a pure couple applied to the crown, the system was in equilibrium when the tipping was resisted by an equal and opposite couple acting on the root. At this point the motion of the tooth ceased and the deflections of the crown and root were recorded on the data sheet.

The rubber material which comprised the analog periodontal 1igament was elastic and homogeneous. Thus, the stresses which resisted the tipping varied at different levels of the root. Theoretically speaking, the maximum stresses occurred at the apex on the side of compression. The minimum stresses occurred at the level of the center of tipping where there were only vertical shear stresses. The point at which the compressive and tensile stresses are zero (under the conditions of a pure couple acting on the crown) is called centroid.

It is significant that the position of the centroid of a body is not affected by changes in the magnitude of the applied couple because the resisting couple will also increase proportionately. Thus, the magnitude of the two forces may be increased, and/or the perpendicular distance between the two forces may be increased and the body will always rotate about the same point (centroid) so long as only a pure couple is acting upon the body. Accordingly, it is possible to predict that, excepting experimental error, the center of tipping would always be the same regardless of the moment of the pure couple applied to the crown of the model tooth.

Experimentally, this was not the case. Figure 23 shows graphically 
the results obtained when pure couples of uniformly increasing magnitudes were applied to the crown. When the moment of the couple was minimal, the tipping occurred about an axis near the apex. As the moment of the couple was increased through the range of 8,12 , and 16 pound-inches, however, the location of the center of tipping remained nearly constant at a point 4.2 inches above the apex. The fact that at the lower magnitudes, the tooth did not behave as predicted, was attributed to the fact that the tooth and its rubber "periodontal ligament" did not fit tightly in the plastic alveolus. At the lower magnitudes all of the strands of rubber did not come into contact with the walls of the alveolus, and the places where the rubber was not tightly compressed against the alveolus could be seen through the clear plastic. At the higher magnitudes, however, the greater stresses that were developed were distributed over more of the surface of the alveolar walls (two "areas of compression" were definitely established) and the tooth conformed to the predicted characteristics.

The deflections of the crown and root, under the influence of pure couples, were in the magnitude of hundredths of a millimeter (see Appendix I). From a study on propagation of error, a variation of less than two-tenths of an inch in the location of the centroid is well within the limits of variation due to experimental error in deflections. It can be stated, therefore, that the centroid was located experimentally to be a level of approximately 4.2 inches above the apex of the model tooth and that tipping occurred consistently about this point when the moment of 
the pure couple applied to the crown was greater than 8 pound-inches. The findings of this part of the experiment substantiated the statement made by Burstone (1962) that "if a pure moment (a couple) is placed anywhere on a tooth, a center of rotation occurs at the centroid of the tooth." He was referring here to the centroid of a two-dimensional representation of a tooth - the point at which the stresses would be zero. He described the position of centroid as being "near the middle of the root." The position of centroid, relative to the middle of the root, was determined more accurately in this study. The total height of the model tooth was 10.6 inches. The height of the root was found by taking the highest point of the cemento-enamel junction on the distal surface and its lowest point on the labial surface and bisecting the distance between these two points. An imaginary plane passing through this bisecting point, parallel to the floor when the tooth is in the upright position, was taken as the upper limit of the root of the tooth. The total length of the root then, from the apex to the average height of the cemento-enamel junction was 7.2 inches. This meant that the centroid, being 4.2 inches above the apex, was not in the exact center of the root. Rather, it was 0.6 inch above the middle of the root.

Haack and Weinstein (1963) had also produced tipping of a model tooth by the application of a clockwise pure couple to the crown. As mentioned in the Review of Literature, their model consisted of an enlarged cross-section of an upper central incisor made of wood. The crosssection was in the labial-1ingual plane and consequently, the tipping 
that occurred under the above-mentioned conditions was in the 1 ingual direction (Figure 10). With respect to the location of the center of rotation (center of tipping) in relation to the height of the root, they observed that "the center of rotation is now a little less than half the root length from the apex." That is to say, they found the center of tipping to be somewhat apical to the center of the root.

The fact that the model tooth used in this research did not behave in exactly the same manner as the model employed by Haack and Weinstein can be explained by the fact that the nature of the two models is entirely different. The model used by Haack and Weinstein had a foam rubber "membrane" of uniform depth as well as uniform thickness. The anatomical model used in this study also had a uniform thickness of rubber webbing throughout, but, because of the contours of the root, there was a greater quantity of rubber around the cervix of the root where the tooth was widest and less rubber surrounding the narrower, apical portion of the root. Consequent1y, the stresses developed were different in the two cases. It is likely that the information provided by the three-dimensional model of a tooth, with the actual contours and configuration of a real tooth was more reliable than that which was derived from the two-dimensional model used by Haack and Weinstein. The two experiments concurred, however, on the finding that "the center of rotation is not affected by variation in the moment of the couple."

Jarabak and Fizze11 (1963) approached the problem of the location of centroid differently. They studied the stresses acting on the root of 
a tooth when only a tipping couple was applied to the crown, and theorized that "the maximum stress occurring on the root can be calculated from the formula for fiber stresses in a beam, which indicates that the maximum stress probably occurs at the apex." This theory will be discussed in detail later in relation to the stresses involved in this research. At this point, therefore, it is relevant only to mention that they labelled the distance from centroid to the most remote periodontal fiber at the apex, a, and the distance from centroid to the extreme fiber in the cervical region of the root, $g$. Since the stresses varied uniformly from the "region of low stress" around the centroid to a maximum at the apex, the distance, a, was greater than the distance, $\mathrm{g}$, which is the same as saying that the centroid was slightly above or cervical to the center of the root when only a pure couple was applied to the crown. This observation has been validated by this investigation.

\section{Assessment of Tipping}

A pure couple is rarely, if ever, applied to a tooth in the mouth because it is virtually impossible to eliminate all the other external forces that act on the tooth. An orthodontic appliance can be designed and constructed to deliver a couple to the crown of a tooth but, invariably, it will also exert some horizontal or vertical or transverse force on the tooth. Even as the activated appliance exerts forces against the bracket, friction develops between the contacting surfaces of the wire and the bracket resulting in frictional forces acting on the tooth. In addition to this consideration, other factors enter in which make the 
matter even more complex. For example, when the tooth is placed in function by chewing or occlusion, other external forces act upon it which tend to negate the existence of a pure couple.

One need only look at the apparatus that was used in this experiment for purposes of applying a pure couple to an enlarged model tooth, to realize how difficult it is to have precise control of all forces acting on a tooth. It is more often the case that tipping is brought about clinically by the action of a single predominating force in the desired direction in combination with other forces that are unavoidable. For this reason, a second method of producing tipping was also selected for study in this investigation.

Single forces ranging in magnitude from one-half pound to five pounds were applied to the model tooth at three different leve1s of the crown. An attempt was made to have the forces perpendicular to the long axis of the tooth by setting the tooth upright in its socket as nearly perpendicular to the floor as possible and the action line of the forces as nearly parallel to the floor as possible. It will be assumed throughout this discussion that all the forces acted at right angles to the long axis of the tooth and that movement of the tooth occurred only in a mesial-distal plane.

The data that were collected in the three trials performed for this part of the experiment were averaged individually (Appendices III, IV, and V) and these averages were plotted as shown in figure 24 . The curves reflect the fact that as the magnitude of the force increased the 
deflections of both the crown and the root also increased. The rate of increase of the deflections was greater at the higher magnitudes although the increments of force magnitude were kept constant at 0.5 pound. As mentioned in Chapter $V$, the deflections of the crown were always in a distal direction while the deflections of the root were in a mesial direction. Naturally, this indicates that in every instance the tooth tipped dista11y about an axis somewhere in the root of the tooth.

The level of the point of application on the crown had definite effects on the degree of tipping produced by a particular force. The graph in figure 24 indicates that both the crown and root deflections were consistently greatest when the forces were applied more incisal1y on the crown (Post $\#_{1}$ ) and least when applied closer to the cervix of the tooth (Post \#4). The reason for this is obvious. When a force, perpendicular to the long axis of the tooth, is applied to the crown, a moment of force is produced which equals the product of the force magnitude multiplied by the moment arm. In the case of the experimental model, the moment arm was the distance between the point of force application and the center of tipping. Thus, a force applied at the top post had the longest moment arm and one applied to the bottom post, the shortest moment arm. One would expect that the greater the moment of force, the greater would be the degree of tipping. This was borne out in the findings.

Earlier it was mentioned that in every instance the tooth tipped 
distally about an axis somewhere in the root. The location of this center of tipping was determined mathematically for each force used as outlined in Appendix II. These equations are based on the established principle of plane geometry that the corresponding sides of two similar triangles are proportional to each other. The diagram in Appendix II is a schematic representation of the long axis of the model tooth and the two dial indicators used in this experiment. The height, $C$, between the two dial indicators was known to be 6.989 inches. The distance between the center of tipping and the root dial indicator was designated, $\mathrm{X}$. Therefore, the distance between the center of tipping and the crown dial indicator was C-X. The crown deflection (a) and root deflection (b) were also known since they were measured experimentally for each force. Hence, it was possible to solve for $X$. Accordingly, it was shown that the distance between the root dial indicator and the center of tipping was equal to 6.989 times the root deflection divided by the sum of the crown and root deflections. To determine the height, $\mathrm{H}$, between the apex and center of tipping, the height, D, (between the root dial indicator and the apex) was added to each value of $\mathrm{X}$.

The location of the center of tipping was plotted against each force that produced the tipping. These data are seen in the graph in figure 25. The different levels of the curves indicate that the center of tipping was consistently furthest from the apex when the forces were applied to the top post. When the forces were applied to the middle post, the center of tipping moved closer to the apex. When the bottom 
post was used as the point of force application, the center of tipping was closest to the apex. This confirmed the statement by Graber (1963) that "several factors influence the position of the fulcrum. One is the point of force application. The closer to the incisal edge the point of force application, the greater the distance of the fulcrum from the apex." Another significant result observed during this part of the investigation was that whenever a single force acted on the crown of the tooth, the center of tipping was always below or more apical to the centroid of effective root area. This was true even for the case of the maximum force applied to the top post. (Tipping occurred about an axis 3.395 inches above the apex when a 5 pound force was applied to the top post.) As mentioned in the previous section, the centroid was found to be at approximately 4.2 inches above the apex, and this was the point about which tipping occurred when a pure couple was applied to the tooth. This finding supports that of Haack and Weinstein (1963) wherein they showed that the center of rotation was located more cervically when a pure couple was applied to the crown in contrast to its more apical position when a single force was used. Burstone (1962) had also found that "a lingually directed single force placed on the crown of a tooth produces a center of rotation somewhere between the centroid and the apex."

The position of the center of tipping did not remain constant for the different force magnitudes. All three curves seen in figure 25 show that as the force increased in magnitude, the center of tipping moved cervically or away from the apex. This progressive shifting of the 
center of tipping relative to the apex occurred throughout the entire range of forces including the higher force magnitudes of 3 , 4, and 5 pounds. This discounted the possibility that the changes in the position of the center of tipping was brought about solely by the fact that the tooth did not fit tightly in its plastic alveolus. Rather, it demonstrated that in this experiment, at least, the force magnitude did have an influence on the location of the center of tipping. Again, this was in agreement with the clinical observation made by Graber (in the work cited above) that "heavy forces move the fulcrum away from the apex; 1ight forces move it closer to the apex."

Although this may be true clinically, certain studies have shown that, theoretically, the magnitude of the force should have no effect on the location of the center of tipping. Burstone (1962) stated in his discussion of the biomechanics of tooth movement that "if a single force were placed on the crown of the tooth, the center of rotation should be the same irrespective of the magnitude of the force. A '1ight' or 'heavy' force would tend equally to move the crown in one direction and the root in the other." of course, his theoretical analysis was based on certain assumptions made about stress distribution in the periodontal 1igament. One of these was that a "linear stress-strain relationship is postulated." In clinical situations, the forces applied to the teeth may exceed the elastic limit of the periodontal fibers or they may even be so excessive that the root of a tooth will come into direct contact with the alveolar crest on one side and the alveolar bone of the fundus 
on the other. In these cases the location of the center of tipping will change as force magnitude is increased. In the analog used in this experiment, apparently a linear stress-strain relationship in the rubber "periodontal ligament" did not exist for the range of forces that was used.

Haack and Weinstein (1963) produced lingual tipping of a wooden model tooth first, by the application of a "light, simple, posteriorly directed force" on the crown and then by applying a "strong, simple force" in the same direction. The point of force application was the same in both cases. They noted that "whereas the amounts of compressions and extensions in the periodontal ligament have changed, the location of the center of rotation has not." This observation was also contrary to the findings of the present study wherein it was found that the center of tipping shifts cervically as force magnitude increases. It was felt that the different nature of the two models must again be held responsible for the disagreement in experimental results.

The model used by Haack and Weinstein had a narrow strip of foam sponge of uniform depth to represent the periodontal ligament around the tooth. The force magnitudes that they used were those that would produce areas of compression and tension in the foam sponge. Even when the "strong, simple force" was used, its magnitude was just enough to produce a slightly greater degree of compression and tension than that which was produced by the lighter force. Under these conditions, a linear stress-strain relationship was possible and the tooth tipped about 
the same point regardless of the increase in force magnitude.

The three-dimensional model used in this research, on the other hand, more closely approximated the actual situation that exists within any real alveolus. That is to say, the cervical portion of the root, being wider than the apical portion, presented a greater surface area and consequently, was surrounded by a greater volume of rubber "periodontal ligament". Therefore, the compressive stresses acting against these portions of the mode1 root were similar to those developed on the side of compression in a real tooth when the latter is being moved distally by a single predominating force in the distal direction. As mentioned above, these compressive stresses in the rubber "periodontal ligament" apparently were not directly proportional to the strains that were produced.

Jarabak and Fizzell (1963) presented roentgenographic evidence to support their assertion that the center of tipping can sometimes 1ie outside of the tooth root. This is certainly true and it can be shown both experimentally and mathematically. This research, however, was not directly concerned with this special type of tipping and hence, discussion of this phenomenon will be deferred until the section on Biophysics of Tooth Movement comes under consideration.

\section{Assessment of Bodily Movement}

A tooth undergoes translation or bodily movement when it changes its position mesially, distally, 1ingually, labially, or buccally, without any change in orientation of its axes. This is often desirable in 
the course of orthodontic treatment, but very difficult to achieve.

This experiment has demonstrated the fact that whenever a single force is applied to a tooth, the tooth tips because of the moment of force that is developed. The same is true when a couple is applied to a tooth. The simplest way of producing pure translation or bodily movement of a free-floating body in space is by applying a single force on the body exactly at its center of mass. The teeth, however, are not freefloating bodies; they are situated and held tightly in a periodontal environment. Consequently, a single force applied on a tooth at the center of mass (if it were possible) would initiate reactive forces against the root of the tooth to resist the movement of the tooth from its rest position. Pure translation would not have necessarily taken place under these conditions. Thus, it is evident that to achieve pure translation of a tooth within its alveolus (or of the model tooth within its plastic alveolus) a single force would have to act through the centroid of effective root area and not through the center of mass of the tooth which may be different. As noted in the Glossary, the former point is the geometric center of the projected area of the root.

Since it is not possible clinically to apply a force exactly at the centroid of effective root area, another method must be employed to produce bodily movement. A force and couple acting simultaneously on the crown of the tooth will produce bodily movement if they are oriented so that, in effect, the resultant is a single force acting through the centroid. 
The various combinations of a force and a couple that meet the requirements necessary to produce pure translation can be calculated mathematically if the position of centroid and the distances between the points of force application are known. The centroid of effective root area was determined in this experiment by applying pure couples to the tooth and observing the point about which the tooth tipped in a mesialdistal plane. This was discussed in a previous section.

Figure 27 shows the vector diagram and the mathematical calculations that were used to determine the necessary dimensions of force and couple that would produce pure translation of the model tooth. The distance, a , between the top and bottom posts on the crown was 1.184 inches. (This was derived by adding heights $\mathrm{E}$ and $\mathrm{F}$ in figure 21.) The position of centroid was taken to be 4.229 inches up from the apex. Further ca1culations give 3.980 inches as the distance between centroid and the bottom post on the crown, which is designated, $\underline{b}$, in figure 27 .

The equations of equilibrium that are applicable to the diagram. are also presented in figure 27. By solving these equations simultaneously and inserting the known values of $\underline{a}$ and $\underline{b}$ in the last step, the following equation results: $F_{m}=1.297 F_{t}$. This indicates that in order for the body in the diagram to be in equilibrium, the magnitude of $F_{m}$ would have to be 1.297 times that of $F_{t}$.

To test these theoretical calculations an experiment was performed on the model tooth as described in Chapter IV (section G.). The experimental results were reported in Chapter $V$ (section $\mathrm{C}$. ). The graph in 
figure 28 shows that as the magnitude of $F_{m}$ increased from 7.5 pounds toward the predicted value of 8.5 pounds the deflections of the crown increased while those of the root decreased. Obviously, the curves in figure 28 would intersect when the deflection of the crown and that of the root were the same value and in the same direction. Experimentally, this occurred, not at the predicted force of 8.577 pounds, but at 8.75 pounds. The error was about 2 percent of the nominal value. This close agreement indicated that the location of the position of centroid had been reliable and that the calculations used to predict the force system necessary to produce pure translation were correct.

The moment of the couple in this system was calculated by multiplying the force $F_{t}$ (6.61 pounds) by the perpendicular distance between $F_{t}$ and $F_{m}\left(1.184\right.$ inches). This is possible because, analytically, $F_{m}$ can be thought of as being two separate forces having the exact same point of application and identical action lines. One of the forces would have a magnitude of 6.61 pounds and, together with $F_{t}$, comprises the couple in the system. The remainder of $F_{m}(8.75-6.61=2.14$ pounds) is the net single force acting on the tooth in a distal direction. Thus it is seen that the actual force system that produced bodily movement of the model tooth a distance of $.034 \mathrm{~mm}$. (Figure 28) in a distal direction consisted of a counter-clockwise couple of 7.81 pound-inches $(6.61 \mathrm{X} 1.184)$ and a single force in the distal direction of 2.14 pounds. Reference to figure 23 shows that when a couple of 7.8 pound-inches is applied to the tooth, the location of centroid was about 4.25 inches 
above the apex. The assumed value had been 4.229 inches.

This experiment with a three-dimensional model tooth confirmed still another empirical observation that had been made by Graber in his textbook on orthodontics that "clinically, with conventional fixed appliances, greater force is usually required for bodily movement". It is obvious now that this is true because of the magnitude of the countercouple that must be exerted on the tooth just to offset the tipping effect of a single force.

Haack and Weinstein (1963) had performed essentially the same experiment on their two-dimensional model. They produced bodily movement of the tooth by applying a single force "so that its action line passes through the center of resistance of the supporting (foam sponge) periodontal ligament." They noted that this center of resistance was the same as the center of rotation obtained when a pure couple had been applied to the model tooth. Having demonstrated qualitatively, the bodily movement produced by a single force acting at the centroid of their model, they then produced the same movement with an equivalent force system that consisted of a couple and a force applied on the crown. They did not attempt, however, to quantitate any of the forces or dimensions used because they felt that "the model and the examples described are chiefly of pedagogic value."

Jarabak and Fizze11 (1963) studied the phenomenon of pure translation from the standpoint of pressure required to start a mandibular right canine moving bodily in a distal direction. They recognized that 
in order to accomplish translation in clinical situations a force and a couple would be necessary because the force system had to be applied at the bracket on the crown of the tooth. The required couple was designated $F_{t}-F_{t}$ and the single force in the distal direction, $F_{m^{\circ}}$ Since the point of application of the resisting force was unknown, it was agreed to be at the centroid of the projected area of the tooth. To determine the location of this centroid they took the dimensions of the mandibular right canine that were shown by Black to be those of an average sized tooth, and then constructed a graph of a parabola that matched closely the outside contours of a mesial-distal projection of the root of this tooth. The equation for this curve was determined by using the dimensions of the actual root to provide the constants. Then through the use of integral calculus, they determined the area contained within the parabola (closed at the top by the $\mathrm{X}$-axis). The value was taken as the projected root area of the tooth and its geometric center or centroid of area was then calculated with integral calculus. With this information they were able to arrive at a reasonable estimate of "the average pressure for best movement." When a practical force value of $250 \mathrm{gm}$. was assigned to $\mathrm{F}_{\mathrm{m}}$, however, and the bracket width was $3.7 \mathrm{~mm}$., they found that the forces inside the slot of the bracket would have to be about $757 \mathrm{gm}$. to provide the counter-couple needed to keep the tooth upright while it translated distally, parallel to the occlusal plane. This magnitude of force is so great and so completely unreasonable for a piece of orthodontic wire of the size that would normally be used, 
that they were led to conclude: "Wlat we have done here is to develop the reason for the customary orthodontic practice of tipping this tooth until its crown is in nearly correct position, then uprighting it in its new position, and finally closing the remaining space between it and the adjacent premolar." In other words, they discounted the feasibility of producing pure, continuous bodily movement by means of current orthodontic appliances. The reasons underlying this clinical fact have been amply demonstrated by this investigation.

\section{Biophysics of Tooth Movement}

In the first part of the Review of Literature, biophysics was defined as the study of biologic function, structure, and organization in relation to and by the methods of physics. The moving of teeth through alveolar bone is certainly a biologic response that is brought about by forces acting on the surfaces of the teeth. These forces are either from the natural environment of the teeth or from orthodontic appliances.

From a clinical viewpoint, two facets of biophysics are of major concern. First, what type of force system is required to produce a given center of tipping, and second, what are the optimal pressures and stresses in the periodontal ligament that stimulate direct resorption of bone? The first of these problems is important because it is evident that if the orthodontist could control the location of the center of tipping, then desired tooth movements could be achieved more directly. This is true even of bodily movement which is tipping about an axis located at infinity. The second consideration is important because, obviously 
teeth would move in a more rapid and expeditious manner, with a minimum of Eamage to the tissues, if the stresses in their respective periodontal ligaments were always optimal. A solution to these problems will require a thorough understanding of forces and moments that may act on the teeth as well as detailed assessment of the histologic changes within the periodontal spaces that are brought about by specific force systems.

This research has thus far answered in part the question of how the center of tipping in a single-rooted tooth is affected by several basic force systems applied to the crown. It has also been established that bodily movement is possible experimentally, although difficult to achieve clinically. The matter of stresses that are developed in the periodontal spaces during the different tooth movements, however, has not been adequately described and must be investigated. This part of the discussion will deal, therefore, with a theoretical evaluation of the stresses that might be produced in the periodontal ligament by each of the force systems used in this study. The analytical thinking that will be used in this section has its basis in the branch of physics called mechanics.

In one respect, the analog used in this investigation was not accurately representative of the anatomical conditions in the periodontal spaces. Since the rubber "periodontal 1 igament" was cemented only to the root surface, it did not reproduce the tensile stresses that exist on the "tension side" of the periodontal ligament. These tensile stresses are probably more important than the compressive stresses in the aratomy. 
Sicher (1962) believes that "the limiting factor in this physiologic orthodontic force is not, as has been claimed, the capillary or tissue pressure, but the tensile strength of the suspensory fibers." The fact that the tensile stresses of the periodontal ligament are replaced by compressive stresses in the analog in no way invalidates the findings of the study. The net effect on the tooth is the same regardless of whether the movement is resisted by a "push" on one side or a "pull" on the other.

It is self-evident that the stresses developed within the periodontal ligament are dependent upon the intensity and the type of force system applied to the crown of the tooth. Essentially, there will always be at least three types of stresses present: (1) compressive stresses on the side of the root where the periodontal ligament is being compressed; (2) tensile stresses on the opposite side where the periodontal fibers are being stretched (the tension side); (3) shearing stresses around the edges of the root and in the regions between areas of pure compression and pure tension. These various stresses cannot readily be separated and measured. Thus, in a quantitative evaluation made by Jarabak and Fizzell (1963) the stresses were "expressed collectively as 'pressure' in grams per square millimeter of projected area." In this theoretical discussion, shearing stresses will be considered negligible and attention will be focused on the tensile stresses that 1 imit the movement of the teeth in clinical situations, and compressive stresses which performed the same function in the analog. The simplifying assumption made by Jarabak and Fizzell to study the phenomenon of stresses will also apply in this 
discussion. That is, the projected root area is assumed to be a close approximation of the effective root area. This is convenient, for it gives the researcher a tool whereby he can calculate the stresses that develop in different situations. This provides a basis for comparison.

It has been established that a tooth will translate or move bodily when the resultant on the tooth is a single force whose action line passes through the centroid of effective root area. Such a case was demonstrated on the model tooth. Since a single force applied to the crown of the tooth would produce a tipping couple, bodily movement was achieved by adding a couple on the crown that would neutralize the moment of the tipping couple. What remained, then, was the translating force $\left(F_{m}\right)$ alone. This brought about the observed bodily movement of $.034 \mathrm{~mm}$. distally that was described earlier.

When translation occurs and the tooth is in equilibrium, the summation of all the resisting forces may be considered as if it were a single force, $F_{r}$, also applied at the centroid of effective root area and equal and opposite to $\mathrm{F}_{\mathrm{m}}$. Under these conditions, a simple relation exists between the translating force and the stress on the projected root area in units of force per unit area. The formula for the stress at any point in the projected root area can be written:

$$
\mathrm{S}=\frac{\mathrm{F}_{\mathrm{m}}}{\mathrm{A}_{\mathrm{r}}}
$$

where $F_{m}$ is the translating force and $A_{r}$ is the effective root area (or projected root area which is considered to the the same). From the section on bodily movement, it was seen that when a force and a couple acted 
on the crown, the net translating force was 2.14 pounds. The projected root area of the model tooth was determined graphically to be $12.68 \mathrm{sq}$. in. The stress in this case was, therefore, $\frac{2.14}{12.68}=.165$ pounds per square inch anywhere on the projected root area.

When a pure couple was applied to the model tooth, it was observed that tipping occurred about the centroid of projected root area. In this instance there was no translating force. Therefore, the stress occurring at any level of the root could be calculated from the formula for fiber stresses in a beam:

$$
\mathrm{S}_{\mathrm{X}}=\frac{\mathrm{MX}}{\mathrm{I}}
$$

$M$ represents the magnitude of the tipping couple, $X$ is the distance from the centroid to the level where the stress is to be determined, and I is the amount of inertia of the projected root area around the axis through the centroid.

Referring once again to the experiment on the model tooth and to figure 23 specifically, it was noted that a couple of 7.8 pound-inches produced tipping about a point approximately 4.25 inches above the apex. (This point was later shown to be the centroid.) To find the stress at the level of the Root Dial Indicator when a couple of 7.8 pound-inches was acting on the crown, the distance $X$ as seen in figure 21 , was determined to be 3.86 inches $(4.25-.390)$. The moment inertia was determined graphically to be 37.99 in. ${ }^{4}$. Substituting these values into the above equation, the stress at the level of the lower dial indicator was found 
to be:

$$
\frac{7.8(3.86)}{37.995}=.792 \text { pounds per square inch. }
$$

To find the stress near the cervix of the root due to a pure couple, the distance $\mathrm{X}$ was taken as 1.91 inches above the centroid. This distance was measured directly on the mode1 tooth with the rubber "periodontal ligament" (See Figure 19). The stress in this cervical region was found to be:

$$
\frac{7.8(1.91)}{37.99}=.380 \text { pounds per square inch. }
$$

When a single force was applied to any of the three posts on the crown, the stresses in the rubber "periodontal 1 igament" were much greater and more complex. As an example, the stress at the level of the Root Dial Indicator was determined for the case of a single force of 5.0 pounds acting on the bottom post (非). The theory of the summation of stresses from two force systems had to be employed because the stresses in this case were neither uniform, as in the case of pure translation, nor uniformly varying, as in the case of pure tipping about centroid. Experimentally it was shown that a single force applied to the crown of the tooth caused the tooth to tip about an axis that was always below or apical to the centroid. If one examines what has occurred to the centroid, itself, in this instance, it becomes obvious that centroid had to shift its position as the tooth tipped about an axis other than centroid. That is, the long axis of the tooth had to undergo a combination of tipping (about centroid) and translation. How this is possible is explained by the principle of mechanics which will be described next. 
Even though only a single force is applied to the crown, this can be resolved into a couple and a force. This is done by imagining that there are two equal and opposite collinear forces, acting at the centroid of projected root area. (Since the resultant of these two imaginary forces is zero they in no way change the real force system applied to the tooth nor alter in any way the equilibrium of the system.) Each of the imaginary forces at centroid is equal to the force applied to the crown. Their action line is parallel with that of the real force applied to the crown. One of these imaginary forces combines with the force on the crown to produce a couple. The other imaginary force acts as a translating force since its action line passes through centroid. The total stress at any point is the algebraic sum of the stresses produced by the force and the couple. Thus, the total stress at the level of the Root Dia1 Indicator is:

$$
S_{X}=\frac{\left(F_{m} b\right) X}{I}-\frac{F_{m}}{A_{r}}
$$

The total stress at the opposite end of the root can be expressed as:

$$
\mathrm{S}_{\mathrm{g}}=\frac{\left(\mathrm{F}_{\mathrm{m}} \mathrm{b}\right) \mathrm{g}}{\mathrm{I}}+\frac{\mathrm{F}_{\mathrm{m}}}{\mathrm{A}_{\mathrm{r}}}
$$

$F_{m}$ in this instance, represents the single force applied to the crown. For this sample calculation of stress, $F_{m}$ will be taken as the 5.0 pound force that was applied to the bottom post in the course of the experiment. $\mathrm{b}$ is the distance from the bottom post to centroid and is equa1 to 3.98 inches (Figure 27). $\mathrm{X}$ is the distance between the centroid and the Root Dial Indicator (Figure 21). As mentioned above, $X=3.86$ inches; $A_{r}$ is the projected root area of the model tooth which was found 
to be 12.68 square inches; and $I$ is the moment of inertia of the projected root area around the axis through centroid $\left(37.99\right.$ in. $\left.^{4}\right)$. $g$ is the distance from the centroid to the level where stress is to be determined in the cervical region of the root. As in the previous example, $g$ will be taken to be 1.91 inches. Two equations were necessary to express the stress in this instance because, according to the theory of superposition, the simultaneous application of both a translating force and a tipping couple causes an addition of the stresses, provided that the maximum stress does not exceed the elastic limit of the stressed material. In order to add tensor quantities like stress, their direction must be taken into consideration. In the situation being discussed here, the stresses due to the translating force and those due to the tipping couple had a cumulative effect in the cervical region and, thus, the algebraic sum was found by adding the two. In the apical portion, however, the stresses due to the tipping couple are, in part, cancelled out by those due to the translating force. Hence, the algebraic sum of the stresses in this case, is found by subtracting the stress due to the translating force from the stress produced by the tipping couple.

Substituting the known values into the equations above, the total stress at the level of the Root Dial Indicator (near the apex) was found to be:

$$
\mathrm{S}_{\mathrm{X}}=\frac{(5.0)(3.98)(3.86)}{37.99}-\frac{5.0}{12.68}=1.628 \text { pounds } \begin{aligned}
& \text { per } \\
& \text { square inch. }
\end{aligned}
$$

The total stress at a leve1 1.91 inches above the centroid (in the cervical region) was found to be: 


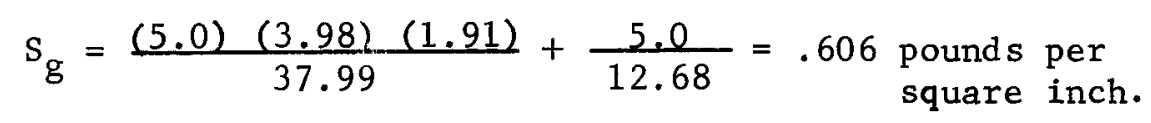

Earlier it was pointed out that a theoretical assessment of the compressive stresses developed in the rubber "periodontal ligament" would be useful because it would provide a basis for comparing the effects that different force systems have on tooth movements. In this light, the actual values of stress presented here become secondary to the following five important observations that can be made. (1) By applying a pure couple to the crown of a tooth, one can obtain tipping around centroid. (2) Pure tipping (about centroid) produces stresses that increase uniformly (from a minimum at centroid) in proportion to the distance away from centroid. The stresses near the apex were greater than those in the cervical region for the simple reason that the apex was further from the centroid. The ratio between these two values of stress was 2.08 (.792 divided by .380). (3) A single force applied to the crown produces a combination of tipping and translation which can be observed both clinically and experimentally (on the model tooth) as a tipping about an axis somewhere between centroid and the apex. The stresses developed in the rubber "periodonta1 1igament" under this condition were also greatest at the apex (on the sicie of compression). The ratio between the stress developed at the apex and that developed at the cervical region in the case of a single force applied to the crown was 2.69 (1.628 divided by .606$)$. This ratio was greater than that for a pure couple (2.08). This indicates that when a single force is applied to 
the crown of a tooth, the apex is more heavily stressed than if a pure couple were used. (4) By complete neutralization of the tipping moment, one can place the center of tipping at infinity and obtain pure trans1ation. (5) This evaluation of stresses in the analog periodontal ligament has provided a method of analytical thinking which might be followed in the future in both animal experiments and clinical investigations. 
CHAPTER VII

\section{SUMMARY AND CONCLUSIONS}

\section{A. Summary}

1. An enlarged, three-dimensional analog of a lower left canine and its periodontal environment was designed and constructed to study the tooth movements that result when various force systems are applied to the crown of the tooth.

2. The force systems applied to the model tooth included pure couples ranging from 2.0 to 16.3 pound-inches and single forces, approximately perpendicular to the long axis of the tooth, ranging from 0.5 to 5.0 pounds. In addition, a combination of a force and a couple was applied to the crown to produce pure translation. The couple in this case was 7.81 pound-inches and the single force was 2.14 pounds.

3. Tooth movements in a mesial-distal plane were measured by two dial indicators that were read to thousandths of a millimeter. One dial indicator measured the deflections of the crown; the other, those of the root.

4. The centroid of effective root area was calculated from the deflections produced by the action of pure couples on the crown. A simple principle of plane geometry served as a basis for these calculations. 
5. The center of tipping was calculated in every instance of tipping produced by the single forces applied at three different levels of the crown. The data were analyzed according to Fisher's Analysis of Variance.

6. It was predicted that bodily movement of the model tooth would result if the force system applied to the crown consisted of a particular force plus a couple. The necessary ratio and magnitudes were calculated mathematically using the position of centroid determined previously. The predicted force system was applied to the tooth. The experimental results showed that the predicted values had been correct to within two percent of the nominal values.

7. A theoretical evaluation of the compressive stresses in the analog periodontal ligament was presented.

B. Conclusions

1. When pure couples were applied to the crown of the model tooth, tipping occurred about a point which became stable when the moment of the couple was equal to or greater than 7.81 pound-inches. Experimentally it can be shown that this definite point is the centroid of effective root area.

2. When single forces were applied to the crown of the model tooth, tipping occurred in every instance about a center of tipping that was somewhere between centroid and the apex. The exact location of this center of tipping can be calculated mathematically if the deflections of the crown and root are known. 
3. The location of the center of tipping was influenced by the magnitude of the applied single force. As the magnitude of the force was increased, the center of tipping shifted further away from the apex, but was always below the centroid. Conversely, when lighter forces were applied to the crown, the center of tipping was consistently closer to the apex.

4. The location of the center of tipping was influenced by the level of the point of force application on the crown. The closer the point of application was to the incisal edge, the further up from the apex was the center of tipping (but always below the centroid). Conversely, as the point of application was moved to positions closer to the cervix, the center of tipping moved closer to the apex.

5. It is possible to predict the force system necessary to produce pure translation if the location of centroid of effective root area is known.

6. It is possible to employ scale models of teeth to investigate tooth movements that take place within the limits of the periodontal spaces when force systems are applied to the crown.

7. An appropriately designed analog of a tooth and its periodontal environment can be used successfully to study the stresses that develop at different levels of the periodontal spaces, and to determine the mathematical calculations and equations of equilibrium that are applicable to real situations in the mouth.

8. Additional studies with physical models, and animal research 
followed by further clinical investigations, will be needed before all the concepts of the biophysics of tooth movement are definitely established and universally understood. 


\section{APPENDIX I}

CROWN AND ROOT DEFLECTIONS PRODUCED BY CLOCKWISE COUPLES ON THE CROWN

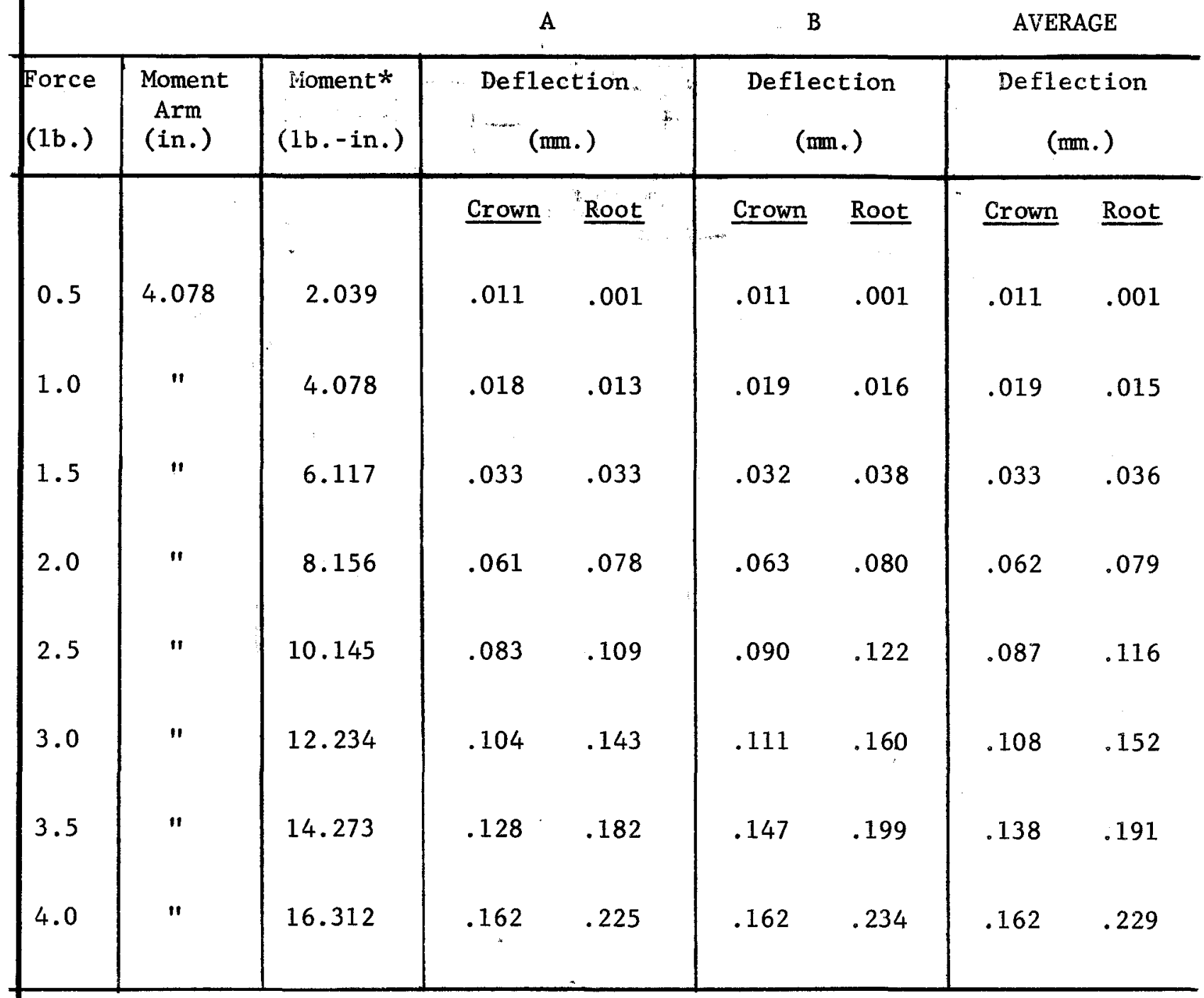

* The moment of force on the steel rod. The magnitude (and direction) of the couple applied to the tooth was equal to this monent of force. 


\section{APPENDIX II}

DIAGRAM OF THE LONG AXIS OF DISTALLY TIPPED TOOTH AND MATHEMATICAL CALCULATIONS FOR DETERMINING LOCATION OF CENTER OF TIPPING IN RELATION TO THE APEX

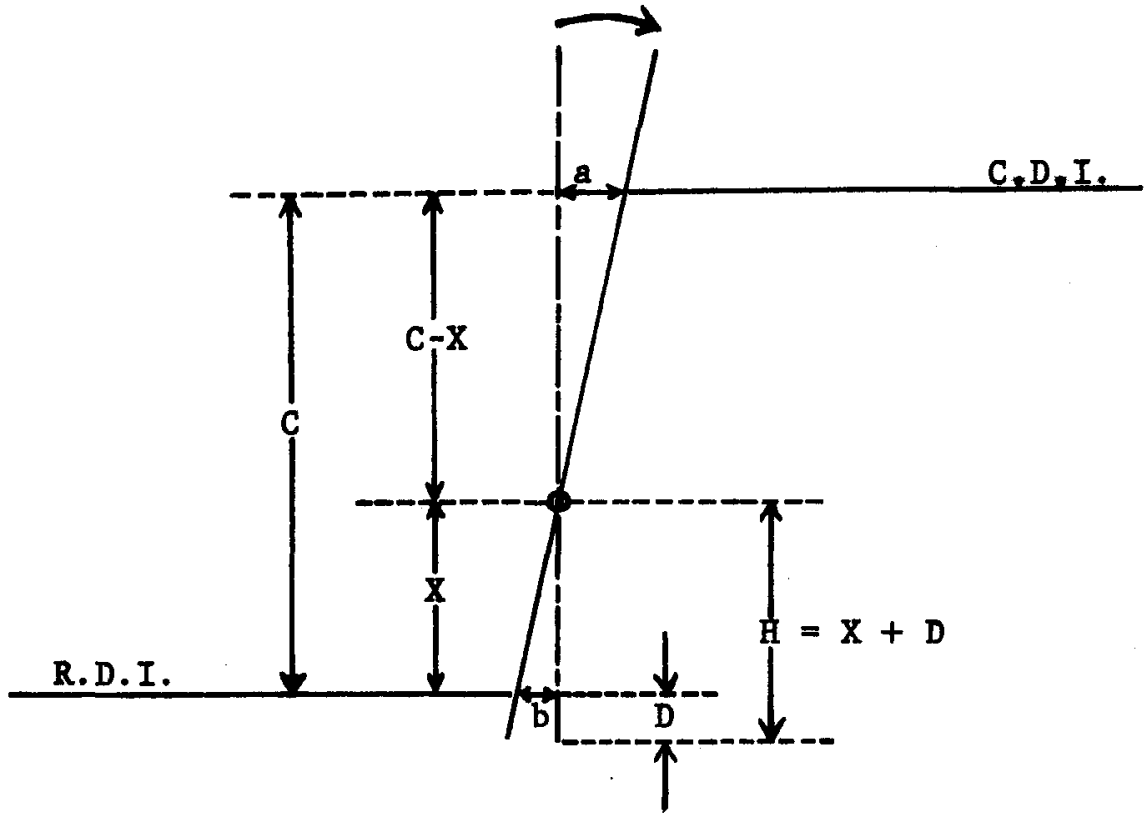
$a=$ crown deflection
$\mathrm{X}: \mathrm{b}: \mathrm{s} \mathrm{C}-\mathrm{X}: \mathrm{a}$
$b=$ root deflection
$a X=b(c-X)$
c $=6.989$ in. (See. Fig. 21)
$a x=b c-b x$
$D=0.390 \mathrm{in}$. (See Fig. 21)
$a X+b X=b c$
$X=$ distance between center of tipping
$x(a+b)=b c$ and Root Dial Indicator
$\mathrm{H}=\mathrm{distance}$ between center of tipping $x=\frac{b c}{a+b}$ 
APPENDIX III

CROWN AND ROOT DEFLECTIONS PRODUCED BY SINGLE

FORCES APPLIED AT THE TOP POST (非)

\begin{tabular}{|c|c|c|c|c|c|c|c|c|}
\hline & A & & B & & C & & AVER & AGE \\
\hline \multirow[t]{2}{*}{$\begin{array}{l}\text { Force } \\
(1 \mathrm{~b} .) \\
\end{array}$} & \multicolumn{2}{|c|}{$\begin{array}{c}\text { Delection } \\
(\mathrm{mm} .) \\
\end{array}$} & \multicolumn{2}{|c|}{$\begin{array}{c}\text { Deflection } \\
(\mathrm{mm} .)\end{array}$} & \multicolumn{2}{|c|}{$\begin{array}{l}\text { Deflection } \\
(\mathrm{mm} .)\end{array}$} & \multicolumn{2}{|c|}{$\begin{array}{c}\text { Deflection } \\
\text { (mm.) }\end{array}$} \\
\hline & Crown & Root & Crown & Root & Crown & Root & Crown & $\underline{\text { Root }}$ \\
\hline 0.5 & .013 & .001 & .016 & .001 & .012 & .003 & .014 & .002 \\
\hline 1.0 & .030 & .013 & .029 & .012 & .025 & .013 & .028 & .013 \\
\hline 1.5 & .050 & .032 & .051 & .033 & .048 & .036 & .050 & .034 \\
\hline 2.0 & .070 & .051 & .071 & .055 & .068 & .054 & .070 & .053 \\
\hline 2.5 & .092 & .081 & .098 & .093 & .096 & .094 & .095 & .089 \\
\hline 3.0 & .118 & .113 & .124 & .123 & .121 & .127 & .121 & .121 \\
\hline 3.5 & .139 & .137 & .149 & .154 & .148 & .152 & .145 & .148 \\
\hline 4.0 & .161 & .163 & .173 & .182 & .174 & .183 & .169 & .176 \\
\hline 4.5 & .200 & .206 & .199 & .208 & .194 & .207 & .198 & .307 \\
\hline 5.0 & .251 & .263 & .245 & .260 & .235 & .256 & .244 & .260 \\
\hline
\end{tabular}


APPEADIX IV

CROWN AND ROOT DEFLECTIONS PRODUCED BY SINGLE

FORCES APPLIED AT THE MIDDLE POST (非)

\begin{tabular}{|c|c|c|c|c|c|c|c|c|}
\hline & A & & I & 3 & c & & $\triangle V E R$ & UAGE \\
\hline \multirow[t]{2}{*}{$\begin{array}{l}\text { Force } \\
\left(1 b_{.}\right)\end{array}$} & \multicolumn{2}{|c|}{$\begin{array}{c}\text { Deflection } \\
\text { (mm.) }\end{array}$} & \multicolumn{2}{|c|}{$\begin{array}{l}\text { Deflection } \\
\text { (mon.) }\end{array}$} & \multicolumn{2}{|c|}{$\begin{array}{l}\text { Deflection } \\
\text { (mm.) }\end{array}$} & \multicolumn{2}{|c|}{$\begin{array}{c}\text { Deflection } \\
\text { (mm.) }\end{array}$} \\
\hline & Crown & Root & Crown & Root & Crown & Root & Crown & Root \\
\hline 0.5 & .018 & .005 & .018 & .002 & .018 & .001 & .018 & .003 \\
\hline 1.0 & .032 & .017 & .032 & .003 & .029 & .010 & .031 & .010 \\
\hline 1.5 & .051 & .034 & .043 & .012 & .048 & .023 & .047 & .023 \\
\hline 2.0 & .069 & .050 & .060 & .026 & .068 & .040 & .066 & .039 \\
\hline 2.5 & .094 & .068 & .079 & .043 & .081 & .051 & .085 & .054 \\
\hline 3.0 & .105 & .076 & .105 & .075 & .114 & .074 & .108 & .075 \\
\hline 3.5 & .131 & .107 & .130 & .101 & .129 & . 099. & .130 & .102 \\
\hline 4.0 & .152 & .135 & .148 & .124 & .142 & .122 & .147 & .127 \\
\hline 4.5 & .183 & .167 & .178 & .152 & .175 & .167 & .179 & .162 \\
\hline 5.0 & .216 & .206 & .210 & .200 & .208 & .201 & .211 & .202 \\
\hline
\end{tabular}




\section{APPENDIX $\quad \mathrm{V}$}

CROWN AND ROOT DEFLECTIONS PRODUCED BY SINGLE

FORCES APPLIED AT THE BOTTOM POST (非)

A

B

C

AVERAGE

\begin{tabular}{|c|c|c|c|c|c|c|c|c|}
\hline \multirow[t]{2}{*}{$\begin{array}{l}\text { Force } \\
\text { (1b.) }\end{array}$} & \multicolumn{2}{|c|}{$\begin{array}{c}\text { Deflection } \\
\left(\mathrm{mm}_{.}\right)\end{array}$} & \multicolumn{2}{|c|}{$\begin{array}{c}\text { Deflection } \\
\text { (mm.) }\end{array}$} & \multicolumn{2}{|c|}{$\begin{array}{c}\text { Deflection } \\
\text { (mm.) }\end{array}$} & \multicolumn{2}{|c|}{$\begin{array}{l}\text { Deflection } \\
\text { (mm.) }\end{array}$} \\
\hline & Crown & Root & crown & Boot & Crown & Root & Crown & Root \\
\hline 0.5 & .015 & .001 & .012 & .001 & .015 & .002 & .014 & .001 \\
\hline 1.0 & .025 & .007 & .023 & .006 & .026 & .006 & .025 & .006 \\
\hline 1.5 & .034 & .013 & .039 & .015 & .042 & .017 & .036 & .015 \\
\hline 2.0 & .052 & .025 & .058 & .027 & .052 & .025 & .054 & .026 \\
\hline 2.5 & .069 & .039 & .074 & .042 & .079 & .042 & .074 & .044 \\
\hline 3.0 & .090 & .058 & .095 & .058 & .104 & .067 & .096 & .061 \\
\hline 3.5 & .111 & .078 & .109 & .074 & .129 & .098 & .116 & .083 \\
\hline 4.0 & .128 & .094 & .134 & .093 & .148 & .114 & .137 & .100 \\
\hline 4.5 & .150 & .113 & .148 & .106 & .168 & .133 & .155 & .117 \\
\hline 5.0 & .188 & .148 & .174 & .144 & .190 & .153 & .184 & .148 \\
\hline
\end{tabular}




\section{GLOSSARY}

ANALOG: That which is analogous to something. It refers to something similar in function but different in origin and structure.

BODY: A quantity of inert matter, the particles of which move little or not at all in relation to one another.

CENTER OF MASS: That point in a (three-dimensional) body about which the sum of the moments of all the individual masses constituting the body is zero.

CENTER OF ROTATION: A fixed point about which a body rotates.

CENTROID: See Centroid of Effective Root Area.

CENTROID OF EFFECTIVE ROOT AREA: The geometric center of the projected root area. The point around which the area is balanced. A line passing through this point, perpendicular to the long axis of the projected root area, bisects the projected root area.

COLLINEAR FORCE SYSTEM: A coplanar force system in which the lines of action lie on the same straight line.

COPLANAR FORCE SYSTEM: A force system in which the lines of action of the forces lie in the same plane.

COUPLE: A system composed of two forces of equal magnitude and opposite direction having lines of action which are parallel but which do not coincide.

EFFECTIVE ROOT PRESSURE: The net motivating force on a tooth divided by the Effective Root Surface Area of that tooth.

EFFECTIVE ROOT SURFACE AREA: That portion of the total surface area of the root that is involved directiy in resisting the movement of a tooth in a specified direction.

EQUILIBRIUM, THE CONDITIONS OF:
a. the sum of the forces in an " $x$ " direction must equal zero.
b. the sum of the forces in a "$y$ " direction must equal zero.
c. the sum of the forces in a " $z$ " direction must equal zero.
d. the sum of the moments about a given point must equal zero. 
FORCE: Any action of one body against another which alters or tends to alter a body's state of rest or of uniform motion in a straight line.

FORCE SYSTEM: A combination of two or more forces acting on a body.

FREE-BODY DIAGRAM: A diagram that shows all of the external forces acting on a body.

FULCRUM: A fixed point against which a lever pushes. Some authors have used this term to mean center of rotation or center of tipping.

LINE OF ACTION: A line of indefinite length of which the force vector is a segment.

MOMENT OF A COUPLE: The product of one of the forces and the perpendicular distance between the forces.

MOMENT OF FORCE: The product of the magnitude of a force, and the perpendicular distance from the line of action of the force to a point which is the center of rotation induced by that force.

PARALLEL FORCE SYSTEM: A force system in which the lines of action are parallel.

POINT OF APPLICATION: The point on a body at which a force is applied. The point of application of a given force acting on a rigid body may be transferred to any other point on the line of action without altering the effect of the force.

PROJECTED ROOT AREA: The area of the projection of the root of a tooth that is made on a screen that is in a plane parallel to the long axis of the tooth when the rays of light are paralle1. For pure translation in the distal direction, the projection that is considered is the one that occurs when the screen is in a buccallingual plane and the light source is in a mesial position directed distally.

STATIC EQUILIBRIUM: The state of a body in which it is experiencing zero acceleration, that is, either moving with a constant velocity or at rest.

STRAIN: Change in volume and/or shape of a body, or part of a body, due to applied forces. The three simplest strains are: (a) longitudinal: change in length per unit length; (b) volume: change in volume per unit volume; (c) shear: angular deformation without change in volume. 
STRESS: The system of forces in equilibrium producing or tending to produce strain in a body or part of a body. Some writers regard the stresses as the force applied to deform the body and others as the equal and opposite forces with which the body resists. In all cases the stress is measured as a force per unit area. The simplest stresses are: (a) tensile or compressive stress, e.g. the force per unit area of cross-section applied to each end of a rod to extend or compress it; (b) hydrostatic pressure, e.g. the force per unit area applied to a body by immersion in a fluid; (c) shear stress, e.g. the system of four tangential stresses applied to the surfaces of a rectangular block (each force being parallel to one edge) tending to strain it so that two of the sides become identical parallelograms, the others being unaltered in shape.

VECTOR: An arrow that is drawn to represent a vector quantity.

,WEIGHT: That force exerted on every body by the gravitational pu11 of the earth. It is the single force which is the resultant of the large number of parallel forces that attract every particle of matter in a body toward the earth. 


\section{BIBLIOGRAPHY}

Adler, P.: The value of model experiments on tooth movement. American Journal of Orthodontics, 32: 583, 1946.

Angle, E.H.: Treatment of the Malocclusion of the Teeth. S.S. White Company, Philadelphia, 1907.

Bauer, W. and Lang, F.J.: Ueber das wandern der zahne. Vierteljahrsschrift fur Zahnheilkunde, 44: 321, 1928.

Boyle, P.E.: In Kronfeld's Histopathology of the Teeth and Their Surrounding Structures, Lea and Febiger, Philadelphia, 1949.

Burstone, C.J., Baldwin, J.J. and Lawless, D.T.: The application of continuous force to orthodontics. Angle Orthodontist, $31: 1,1961$.

Burstone, C.J.: The biomechanics of tooth movement. In Kraus, B.S. and Riede1, R.A.: Vistas in Orthodontics. Lea and Febiger, Philadelphia, 1962.

Case, C.S.: Dental Orthopedia and Prosthetic Correction of Cleft Palate. C.S. Case Company, Chicago, 1921.

Dempster, W.T. and Duddles, R.A.: Tooth statics: equilibrium of a freebody. Journal of the American Dental Association, 68: 652, 1964.

Drenker, E.W,: Forces and torques associated with second order bends. American Journal of Orthodontics, 42: 766, 1956.

Fickel, W.: Die drehachse bei einwurzligen zahnen und deren anderung bei verschiedenen belastungsrichtungen. Deutsche Monatschrift fur Zahnheilkunde, 48: 1137, 1930.

Fish, G.D.: Technology in orthodontia. International Journa1 of Orthodontia, 3: 324, 1917.

Follico, E.S.: M.S. Thesis, Loyola University School of Dentistry, Chicago, 1964.

Gantt, J.P.: M.S. Thesis, Loyola University School of Dentistry, Chicago, 1960. 
Graber, T.M.: Orthodontics - Principles and Practice. W.B. Saunders Company, Philadelphia and London, 1961, reprinted November 1963.

Gysi, A.: Resiliency and the like in its effect on the facet angulations of axtificial teeth. Dental Digest, 36: 623, 1930.

Haack, D.C. and Weinstein, S.: Geometry and mechanics as related to tooth movement studied by means of two-dimensional mode1. Journal of the American Dental Association, 66: 157, 1963.

Hanau, R.L.: Orthodontic mechanics: dental engineering. International Journal of Orthodontia, 3: 410, 1917.

Hay, G.E.: The equilibrium of a thin compressible membrane. Canadian Journal of Research, 17 (A): 106, 1939.

-.--. The equilibrium of a thin compressible membrane with application to the periodontal membrane. Canadian Journal of Research, 17 (A) : 123,1939 .

----. Ischemia of the periodontal membrane. Oral Health, 29: 423, 1939.

Isaacson, J.D. and Ledley, R.S.: Tooth statics. Journa1 of the American Denta1 Association, 69: 805, 1964.

Jarabak, J.R. and Fizze11, J.A.: Technique and Treatment with the Light Wire Appliance; Light Differential Forces in C1inical Orthodontics. C.V. Mosby Company, St. Louis, 1963.

Johnson, A.L., Appleton, J.L. and Rittershofer, L.S.: Tissue changes involved in tooth movement. International Journal of Orthodontia, 12: 889, 1926 .

Kemp, K.I.: M.S. Thesis, Loyola University School of Dentistry, Chicago, 1961.

Kravica, R.E.: M.S. Thesis, Loyola University School of Dentistry, Chicago, 1963.

Kronfeld, R.: Histologic study of the influence of function on the human periodontal membrane. Journal of the American Dental Association, 18: 1242, 1931 .

---- Histopathology of the Teeth and Their Surrounding Structures. Lea and Febiger, Philadelphia, 1949.

MacEwan, D.C.: Treatment of a typical distocclusion case. American Journal of Orthodontics, 40:350, 1954. 
Oppenheim, A.: Tissue changes, particularly of the bone incident to tooth movement. Supp1. to Oesterreichisch-ungarischen Vierteljahrsschrift fur Zahnheilkunde, 27: 303, 1911.

Renfroe, E.W.: The source of power. The Bulletin of the National Dental Association, 16: 16, 1951.

Richmond, J.E.: An appliance analysis with measured pressure.

International Journal of Orthodontia and Dentistry for Children, 19: 898, 1933 .

Sandstedt, C.: Einige beitrage zur theorie der zahnregulierung. Nordisk Tandlakare Tidsskrift, No. 4, 1904: Nos. 1 and 2, 1905.

Schwarz, A.M.: Ueber die bewegung belasteter zahne. Zeitschrift fur Stomatologie, 26: 40, 1928.

----. Mathematische uberprufung des verhaltens belasteter zahne. Zeitschrift fur Stomatologie, 26: 398, 1928.

--.-. Tissue changes incident to orthodontic treatment. International Journal of Orthodontia, Oral Surgery and Radiography, 18: 331, 1932.

Shroff, F.R.: The behavior of collagen fibers in some types of periodontal disease. Journal of Oral Surgery, Oral Medicine and Oral Pathology, 6: 1202, 1953.

Shwa1b, R.A. and Rechter, A.: Application of the photoelastic method of stress analysis to orthodontic tooth movement in bone. American Journal of Orthodontics, 36: 192, 1950.

Sicher, H.: The function of the periodontal ligament. In Kraus, B.S. and Riedel, R.A.: Vistas in Orthodontics. Lea and Febiger, Philadelphia, 1962.

Stanton, F.I.: Engineering in orthodontic diagnosis. Journal of the American Dental Association, 15: 825, 1928.

Stier, E.F.: M.S. Thesis, Loyola University School of Dentistry, Chicago, 1962 .

Stoller, A.E.: Logic in clinical application of differential 1ight forces. Read before the American Association of Orthodontists, Chicago, Illinois, May 4, 1964. 
Storey, E. and Smith, R.: Force in orthodontics and its relation to tooth movement. The Australian Journal of Dentistry, 56: 11, 1952.

Synge, J.L.: The tightness of teeth, considered as a problem concerning the equilibrium of a thin incompressible elastic membrane. Philosophical Transactions of the Royal Society of London, Series A., 231: 435, 1933.

-..-. The theory of an incompressible periodontal membrane. Internationa1 Journa1 of Orthodontia and Dentistry for Children, 19: $567,1933$.

Teasley, G.H.,Jr., Penley, W.E. and Morrison, W.E.: Research project presented to the Postgraduate School of the University of Texas Dental Branch, Houston, 1962.

Weinstein, S. and Haack, D.C.: Theoretical mechanics and practical orthodontics. Angle Orthodontist, 29: 177, 1959.

Weinstein, S., Haack, D.C., Morris, L.Y., Snyder, B.B. and Attaway, H.E.: On an equilibrium theory of tooth position. Angle Orthodontist, 33: 1,1963 .

Zak, B.: Die photoelastische analyse in der orthodontischen mechanik. Zeitschrift fur Stomatologie, 33: 22, 87, 1935. 
APPROVAL SHEET

The thesis submitted by Dr. Edgar F. Geiger has been read and approved by members of the Department of Oral Biology.

The final copies have been examined by the director of the thesis and the signature which appears below verifies the fact that any necessary changes have been incorporated and that the thesis is now given final approval with reference to content, form, and mechanical accuracy.

The thesis is therefore accepted in partial fulfillment of the requirements for the Degree of Master of Science.
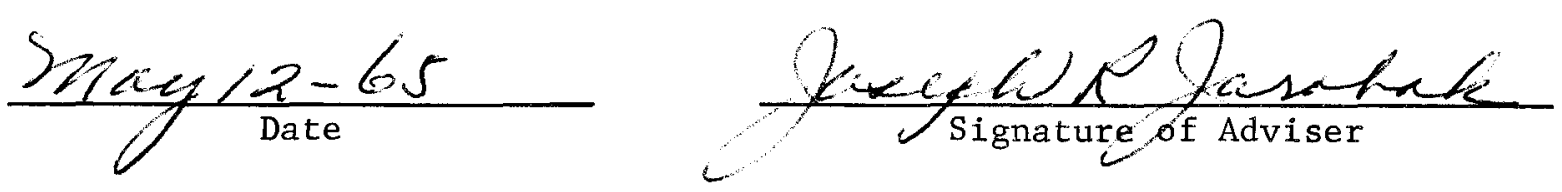